\title{
Estudo da Estabilidade de Soluções de uma Classe de Equações Diferenciais com Retardamento e Aplicações
}

\author{
Marta Cilene Gadotti
}

Orientadora: Profa. Dra. Sandra M. S. de Godoy

Dissertação apresentada ao Instituto de Ciências Matemáticas de São Carlc da Universidade de São Paulo, como parte dos requisitos para obtenção do título "Mestre em Ciências - Área: Matemática".

USP - São Carlos

Fevereiro de 1998 
Aos meus pais e ao Valdir 


\section{AGRADECIMENTOS}

À Profa Sandra Maria Semensato de Godoy, pela dedicação, atenção e assistência, com que empregou neste trabalho.

Ao Prof. Hermínio Cassago Junior, pela importante colaboração, pelas sugestões que contribuíram com este trabalho e pela forma simpática e atenciosa que sempre nos atendeu.

Ao meu amigo Márcio, pela colaboração e pelo incentivo durante estes anos.

À família, pela credibilidade e compreensão ao longo destes anos.

A todos os professores que contribuíram na realização deste trabalho, em especial a Ires Dias, Janey Antonio Daccach, Alexandre Nolasco de Carvalho, José Luis Arraut Vergara, Valdir Antonio Menegatto, Janete Crema, Alberto Leon Krusher (professor visitante) do ICMSC-USP. Também, Nativi Viana Pereira Bertolo, Rosa Lucia Sverzut Baroni, Solange Mancini e Francisco Gilberto Loibel, do IGCEUNESP, Rio Claro, pelo incentivo e pela forma atenciosa que sempre nos atenderam.

A todos os amigos que nos apoiaram durante estes anos, em especial à Katia e à Regilene, pelo companherismo durante os anos de graduação e pós-graduação.

A CAPES, pelo auxílio financeiro empregado neste trabalho.

A todos os funcionários do ICMSC-USP, pela disposição e pela forma atenciosa com que nos atenderam.

Enfim, a todos que direta ou indiretamente contribuíram para a conclusão deste trabalho. 


\section{ABSTRACT}

The changes in the stability of a class of linear differential delay equations are studied, by taking the delay as a parameter.

Conditions assuring stability or instability of steady-state equilibrium solutions are presented.

Finally, some applications on biological and physical models are made. 


\section{RESUMO}

As trocas na estabilidade de uma classe de equações diferenciais lineares com retardamento são estudadas, quando se considera o retardo como um parâmetro.

Condições garantindo estabilidade ou instabilidade das soluções de equilíbrio são apresentadas.

Finalmente, algumas aplicações a modelos biológicos e físicos são feitas. 


\section{Índice}

Introdução

1 Preliminares

2 Estudo da estabilidade de soluções

3 Estabilidade Absoluta

4 Uma aplicação interessante 55

5 Aplicações

68

Bibliografia

89 


\section{Introdução}

Este trabalho foi desenvolvido com o propósito de estudarmos as possíveis trocas de estabilidade de soluções de certas equações diferenciais lineares com retardamento, do tipo:

$$
\sum_{k=0}^{n} a_{k} \frac{d^{k}}{d t^{k}} u(t)+\sum_{k=0}^{m} b_{k} \frac{d^{k}}{d t^{k}} u(t-T)=0 .
$$

Fomos motivados pela importância de tais tipos de equações, já que aparecem em diversos problemas, presentes em vários campos da ciência, como por exemplo na Física, na Biologia e na Ecologia. Veja [4], [5], [7], [12], [13], [15], [16], [17] e [18].

Para estudar o comportamento assintótico de soluções de equações do tipo (1), é necessário analisar a localização das raízes no plano complexo, da equação característica associada. E o tipo mais simples de tais equações tem a seguinte forma:

$$
P(z)+Q(z) e^{-T z}=0
$$

onde $P$ e $Q$ são, em geral, polinomiais de graus $n$ e $m$, respectivamente e $T$ uma constante não negativa.

O trabalho centralizou-se no artigo [3], o qual traz um importante resultado para uma classe de equações do tipo (2), levando-se em conta a localização das raízes de (2), como uma função do parâmetro $T, 0 \leq T<\infty$. Em vista deste resultado, foi examinada três possibilidades:

(i) a equação (2) possui uma raiz em $\operatorname{Re}(z) \geq 0$ para todo $T$;

(ii) existe um número finito de valores de $T$ para os quais as raízes de (2) podem ou não estar em $\operatorname{Re}(z) \geq 0$, mas para todo $T$ suficientemente grande, a equação (2) tem uma raiz em $\operatorname{Re}(z) \geq 0$;

(iii) todas as raízes de (2) estão em $\operatorname{Re}(z)<0$, para $0 \leq T<\infty$.

No Capítulo 1, trazemos as definições e resultados básicos sobre a Teoria Geral das Equações Diferenciais Funcionais com Retardamento, além de outros fatos 
básicos que foram necessários para atingirmos os nossos objetivos.

No Capítulo 2, nos preocupamos em realizar uma demonstração minuciosa do Teorema Principal, cuja demonstração não aparece em detalhes em [3], e, para isso, foi necessário fazer uma análise detalhada da estabilidade de soluções de três equações diferenciais com retardamento de segunda ordem:

$$
\begin{gathered}
\frac{d^{2} x}{d t^{2}}+a \frac{d x}{d t}+b \frac{d x}{d t}(t-T)+c x(t)=0, \\
\frac{d^{2} x}{d t^{2}}+a \frac{d x}{d t}+b x(t)+c x(t-T)=0, \\
\frac{d^{2} x}{d t^{2}}+a \frac{d x}{d t}+b \frac{d x}{d t}(t-T)+c x(t)+d x(t-T)=0 .
\end{gathered}
$$

Observamos que as equações características associadas a estas equações diferenciais retardadas são casos particulares de (2).

Neste capítulo, ainda apresentamos um resultado para equações que apresentam vários retardos e cuja equação característica é do tipo:

$$
\sum_{j=0}^{L} P_{j}(z) e^{-\tau_{j} z}+Q(z) e^{-T z}=0
$$

onde $0=\tau_{0}<\tau_{1}<\cdots<\tau_{L}$ e $T \geq 0$.

No Capítulo 3, inspirados em [2], abordamos o conceito de estabilidade absoluta. Utilizando a teoria apresentada no Capítulo 2, estudamos um outro resultado, que envolve uma condição necessária e suficiente para a estabilidade absoluta de uma equação do tipo (2).

No Capítulo 4, motivados por [5], fizemos um estudo completo de um sistema de equações diferenciais com retardamento, o qual controla o mecanismo da respiração nos seres humanos, utilizando, para isto, a teoria do Capítulo 2.

No Capítulo 5, fizemos diversas aplicações dos resultados obtidos nos Capítulos 2 e 3 a modelos matemáticos, que surgem em bilologia e física. 


\section{Capítulo 1}

\section{Preliminares}

Neste capítulo introduziremos algumas definições e resultados referentes à teoria básica das equações diferenciais funcionais, necessários para compreendermos este trabalho.

Definição 1.1. Se $r \geq 0$ então denotamos por $C=C[-r, 0]$ o espaço de Banach das funções contínuas de $[-r, 0]$ em $\mathbb{R}^{n}$ e consideramos neste espaço a seguinte norma:

$$
|\Phi|=\sup _{-r \leq \theta \leq 0}|\Phi(\theta)|
$$

para $\Phi \in C$. Se $\sigma \in \mathbb{R}, A \geq 0$ e $x \in C\left([\sigma-r, \sigma+A], \mathbb{R}^{n}\right)$, entâo para qualquer $t \in[\sigma, \sigma+A]$, definimos a aplicação $x_{t} \in C$, por

$$
x_{t}(\theta)=x(t+\theta), \quad \text { para } \quad-r \leq \theta \leq 0 .
$$

Se $D \subset \mathbb{R} \times C$ e $f: D \longrightarrow \mathbb{R}^{n}$, dizemos que a relação

$$
\dot{x}(t)=f\left(t, x_{t}\right)
$$

é uma equação diferencial funcional com retardamento sobre $D$ e denotaremos (1.1) por $\operatorname{EDFR}(f)$.

Definição 1.2. Uma função $x$ é uma solução da equação (1.1) em $[\sigma-r, \sigma+A)$ se existirem $\sigma \in \mathbb{R}$ e $A>0$, tais que $x \in C\left([\sigma-r, \sigma+A), \mathbb{R}^{n}\right),\left(t, x_{t}\right) \in D$ ex $x(t)$ satisfaz (1.1) para $t \in[\sigma, \sigma+A)$.

Dados $\sigma \in \mathbb{R}, \Phi \in C$, dizemos que $x(t, \Phi, f)$ é uma solução da equação (1.1) com valor inicial $\Phi$ em $\sigma$ se existir $A>0$ tal que $x(\sigma, \Phi, f)$ é uma solução de (1.1) em $[\sigma-r, \sigma+A)$ e $x_{\sigma}(\sigma, \Phi, f)=\Phi$. 
Quando $r=0$ na definição acima, obtemos uma equação diferencial ordinária.

Seguindo estas notações vamos considerar alguns importantes resultados, enunciados a seguir, que podem ser encontrados em [11].

Lema 1.1. Sejam $\sigma \in \mathbb{R}, \Phi \in C$ e $f(t, \Phi)$ uma função contínua, então encontrar uma solução da equação (1.1) com valor inicial $(\sigma, \Phi)$ é equivalente a resolver a equação integral:

$$
\left\{\begin{array}{l}
x_{\sigma}=\Phi \\
x(t)=\Phi(0)+\int_{\sigma}^{t} f\left(s, x_{s}\right) d s, \quad t \geq \sigma .
\end{array}\right.
$$

Este resultado foi utilizado nas demonstrações dos teoremas seguintes.

\section{Teorema 1.1. (Existência)}

Suponhamos que $\Omega$ seja um conjunto aberto em $\mathbb{R} \times C, f^{0} \in C\left(\Omega, \mathbb{R}^{n}\right)$ dada. Se $(\sigma, \Phi) \in \Omega$, então existe uma solução da $\operatorname{EDFR}\left(f^{0}\right)$ que passa por $(\sigma, \Phi)$. Mais geralmente, seja $W \subseteq \Omega$ compacto e $f^{0} \in C\left(\Omega, \mathbb{R}^{n}\right)$ dada, então existe uma vizinhança $V \subseteq \Omega$ de $W$ tal que $f^{0} \in C^{0}\left(V, \mathbb{R}^{n}\right)$; existe uma vizinhança $U \subseteq C^{0}\left(V, \mathbb{R}^{n}\right)$ de $f^{0}$ e $\alpha>0$ tal que, para qualquer $(\sigma, \Phi) \in W, f \in U$, existe uma solução $x(\sigma, \Phi, f)$ da $\operatorname{EDFR}(f)$ que passa por $(\sigma, \Phi)$ definida em $[\sigma-r, \sigma+\alpha]$.

\section{Teorema 1.2. (Dependência Contínua)}

Suponhamos que $\Omega$ seja um conjunto aberto em $\mathbb{R} \times C, f^{0} \in C\left(\Omega, \mathbb{R}^{n}\right),\left(\sigma^{0}, \Phi^{0}\right) \in$ $\Omega$ e $x^{0}$ é uma solução da $E D F R\left(f^{0}\right)$ que passa por $\left(\sigma^{0}, \Phi^{0}\right)$ a qual existe e é única sobre $\left[\sigma^{0}-r, b\right]$. Seja $W^{0} \subseteq \Omega$ um conjunto compacto definido por

$$
W^{0}=\left\{\left(t, x_{t}^{0}\right) ; t \in\left[\sigma^{0}, b\right]\right\}
$$

e seja $V^{0}$ uma vizinhança de $W^{0}$ na qual $f^{0}$ é limitada. Se $\left(\sigma^{k}, \Phi^{k}, f^{k}\right), k=$ $1,2,3, \ldots$ satisfaz $\sigma^{k} \rightarrow \sigma^{0}, \Phi^{k} \rightarrow \Phi^{0} e\left|f^{k}-f^{0}\right|_{V^{0}} \rightarrow 0$, quando $k \rightarrow \infty$, então existe um $k^{0}$ tal que a EDFR $\left(f^{k}\right)$ para $k \geq k^{0}$ é tal que cada solução $x^{k}=\left(\sigma^{k}, \Phi^{k}, f^{k}\right)$ que passa por $\left(\sigma^{k}, \Phi^{k}\right)$ existe em $\left[\sigma^{k}-r, b\right]$ e $x^{k} \rightarrow x^{0}$ uniformemente em $\left[\sigma^{0}-r, b\right]$. Por $x^{k} \rightarrow x^{0}$ uniformemente em $\left[\sigma^{0}-r, b\right]$, entendemos que, para qualquer $\epsilon>0$, existe um $k_{1}(\epsilon)$ tal que $x^{k}(t), k \geq k_{1}(\epsilon)$, é definida sobre $\left[\sigma^{0}-r+\epsilon, b\right]$, e $x^{k} \rightarrow x^{0}$ sobre $\left[\sigma^{0}-r+\epsilon, b\right]$. 
Teorema 1.3. (Unicidade)

Sejam $\Omega$ um conjunto aberto em $\mathbb{R} \times C, f: \Omega \rightarrow \mathbb{R}^{n}$ uma função contínua, $f(t, \Phi)$ lipschitziana em $\Phi$ em cada conjunto compacto de $\Omega$. Se $f(\sigma, \Phi) \in \Omega$, então existe uma única solução da equação (1.1) que passa por $(\sigma, \Phi)$.

A definição que segue abaixo pode ser encontrada em [11].

Definição 1.3. Seja $f: \mathbb{R} \times C \longrightarrow \mathbb{R}^{n}$ contínua e considere a $E D F R(f)$ :

$$
\dot{x}(t)=f\left(t, x_{t}\right)
$$

Seja $f$ completamente contínua e que satisfaça as condições de igualdade adicionais suficientes para garantir que a solução $x(\sigma, \Phi)$ que passa por $(\sigma, \Phi)$ seja contínua em $(\sigma, \Phi, f)$ no domínio de definição da função. Suponhamos que $f(t, 0)=0$, para qualquer $t \in \mathbb{R}$. A solução $x=0$ da equação acima é dita ser estável se para qualquer $\sigma \in \mathbb{R}, \epsilon>0$, existir um número $\delta>0, \delta=\delta(\epsilon, \sigma)$ tal que $\Phi \in B(0 ; \delta)$, onde, por $B(0 ; \delta)$ denotamos a bola aberta contida em $C$ com centro em 0 e raio $\delta$, implica que $x_{t}(\sigma, \Phi) \in B(0 ; \epsilon)$, para $t \geq \sigma$.

A solução $x=0$ é dita ser assintoticamente estável se ela for estável e se existir um $b_{0}=b_{0}(\sigma)>0$, tal que $\Phi \in B\left(0 ; b_{0}\right)$ implica que $x_{t}(\sigma, \Phi)(t) \longrightarrow 0$ quando $t \longrightarrow \infty$.

$A$ solução $x=0$ é dita ser uniformemente estável se o número $\delta$ da definição for independente de $\sigma$.

$A$ solução $x=0$ é dita ser uniformemente assintoticamente estável se for uniformemente estável e se existir um $b_{0}>0$ tal que para todo $\eta>0$, deve existir $b_{0}(\eta)$ tal que, se $\Phi \in B\left(0 ; b_{0}\right)$ implicar $x_{t}(\sigma, \Phi) \in B(0 ; \eta)$, para $t \geq \sigma+b_{0}(\eta)$ para qualquer $\sigma \in \mathbb{R}$.

Seja $y(t)$ uma solução qualquer da $\operatorname{EDFR}(\mathrm{f})$, então $y$ é dita ser estável se a solução nula, $z=0$, da equação

$$
\dot{z}(t)=f\left(t, z_{t}+y_{t}\right)-f\left(t, y_{t}\right)
$$

for estável. Os outros conceitos são definidos similarmente.

Vamos também utilizar os seguintes resultados de Análise Complexa, que podem ser encontrados em [6]. 
Teorema 1.4. (Teorema de Rouché)

Sejam $F(z)$ e $G(z)$ duas funções analíticas definidas em um domínio $D$, simples, conexo e contendo um contorno de Jordan C. Seja $|F(z)|>|G(z)|$ sobre C. Então $F(z)$ e $F(z)+G(z)$ têm o mesmo número de zeros em $C$.

Teorema 1.5. (Princípio do Máximo)

Seja $F(z)$ uma função analítica em uma região limitada $D$, e seja $|F(z)|$ contínua na região fechada $\bar{D}$. Então $|F(z)|$ assume seu máximo na fronteira da região. 


\section{Capítulo 2}

\section{Estudo da estabilidade de soluções}

Consideremos a equação diferencial linear com retardamento

$$
\sum_{k=0}^{n} a_{k} \frac{d^{k}}{d t^{k}} u(t)+\sum_{k=0}^{m} b_{k} \frac{d^{k}}{d t^{k}} u(t-T)=0
$$

Observemos que uma condição necessária e suficiente para existência de uma solução não trivial de (2.1) da forma $u(t)=c e^{T z}$, onde $c$ é uma constante real, é que $z$ seja uma raiz da equação

$$
P(z)+Q(z) e^{-T z}=0,
$$

onde $P$ e $Q$ são polinômios com coeficientes reais de grau $n$ e $m$, respectivamente, e $T$ uma constante não negativa.

Definição 2.1. A equação (2.2) é chamada equação característica associada à equação (2.1).

O próximo resultaḍo afirma que a estabilidade de soluções de equações diferenciais com retardamento depende da localização dos zeros da equação característica associada.

Teorema 2.1. A solução nula de (2.1) é assintóticamente uniformemente estável se, e somente se, todos os zeros da equação característica (2.2) estão no semi plano esquerdo, isto é, $\operatorname{Re}(z)<0$.

A demonstração desse resultado pode ser encontrada em [1].

Como nosso objetivo é analisar a estabilidade, uma técnica padrão para isto é determinar o equilíbrio da equação ou do sistema de equações diferenciais com 
retardamento, em seguida linearizá-lo em torno do equilíbrio. Por equilíbrio de um sistema de equações diferenciais com retardamento queremos dizer simplesmente uma solução constante; assim os equilíbrios são encontrados fazendo o lado direito de cada equação do sistema igual a zero. A linearização de um sistema em torno do equilíbrio é obtida utilizando-se o Teorema de Taylor e conservando-se apenas os termos lineares. E daí, a equação característica associada ao sistema é determinada expressando-se a condição de que o sistema linearizado possue uma solução que é múltiplo constante de $e^{z T}$, onde $T$ representa o retardo.

Neste capítulo, provaremos o resultado principal de nosso trabalho, o qual fornece condições para analisarmos as trocas de estabilidade das soluções de equações do tipo (2.1), que podem ocorrer quando consideramos o retardo $T$ como parâmetro. Conforme $T$ varia, pode ocorrer que zeros da equação característica associada cruzem o eixo imaginário e a solução da equação mude de estável para instável ou vice-versa. Neste caso, dizemos que ocorre uma troca de estabilidade.

Abaixo, segue o resultado central deste trabalho, o qual será demonstrado com detalhes.

Teorema 2.2. Consideremos a equação (2.2), onde $P$ e $Q$ são funçôes analíticas no semi-plano direito $\operatorname{Re}(z)>-\delta, \delta>0$. Suponhamos que as seguintes condições estejam satisfeitas:

(i) $P(z)$ e $Q(z)$ não possuem zeros imaginários comuns;

(ii) $\overline{P(-i y)}=P(i y)$ e $\overline{Q(-i y)}=Q(i y)$, para $y$ real;

(iii) $P(0)+Q(0) \neq 0$;

(iv) Existe no máximo um número finito de raízes de (2.2) no semi-plano direito quando $T=0$; finito de zeros reais.

(v) $F(y)=|P(i y)|^{2}-|Q(i y)|^{2}$, para y real, tem no máximo um número

Sob essas condições as seguintes afirmações são verdadeiras:

a) Suponhamos que a equação $F(y)=0$ não tenha raizes positivas. Então, se (2.2) for estável em $T=0$, ela permanece estável para todo $T \geq 0$, e se for instável em $T=0$, permanece instável para todo $T \geq 0$;

b) Suponhamos que $F(y)=0$ tenha pelo menos uma raiz positiva e que cada raiz positiva seja simples. Então, conforme $T$ cresce, trocas de estabilidade podem ocorrer, e existe um número positivo $T^{*}$ tal que a equação (2.2) é instável para $T>T^{*}$, e, conforme $T$ varia de 0 a $T^{*}$, no máximo um número finito de trocas 
de estabilidade podem ocorrer.

Para provarmos este Teorema, vamos precisar desenvolver vários resultados.

Note que é possível, a partir de uma solução imaginária pura iy de (2.2), determinarmos valores de $T$ que satisfaçam (2.2). Se $z=i y, y \neq 0$, for uma raiz de (2.2), e veremos que podemos escolher $y>0$ sem perda de generalidade, então $P(i y)+Q(i y) e^{-T i y}=0$, implica

$$
|P(i y)|=|Q(i y)|
$$

e (2.3) determina um conjunto de valores possíveis para y. Escrevendo

$$
\begin{aligned}
& P(i y)=P_{R}(y)+i P_{I}(y) \\
& Q(i y)=Q_{R}(y)+i Q_{I}(y),
\end{aligned}
$$

onde $P_{R}, P_{I}, Q_{R}$ e $Q_{I}$ são funções de $y$ a valores reais, podemos reescrever (2.2) da seguinte forma:

$$
P_{R}(y)+i P_{I}(y)+\left[Q_{R}(y)+i Q_{I}(y)\right](\cos (T y)-i \operatorname{sen}(T y))=0 .
$$

Igualando partes real e imaginária, em (2.5), obtemos:

$$
\left\{\begin{array}{l}
\cos (T y)=\frac{-\left(P_{R} Q_{R}+P_{I} Q_{I}\right)}{Q_{R}^{2}+Q_{I}^{2}} \\
\operatorname{sen}(T y)=\frac{-P_{R} Q_{I}+P_{I} Q_{R}}{Q_{R}^{2}+Q_{I}^{2}}
\end{array}\right.
$$

Veremos, pelo resultado abaixo, que não é possível que $Q_{R}$ e $Q_{I}$ sejam ambos nulos. Assim, para cada raiz $y$ de (2.3), é possível determinar valores de $T$ que satisfaçam (2.6). Como as funções seno e cosseno são periódicas obtemos infinitos valores de $T$.

Suponhamos então, que iy e $T$ satisfaçam (2.2), (2.3) e (2.6). Consideremos as raízes $z=x+i y$ de (2.2) como uma função de $T$, e vamos determinar a direção $s$ do movimento de $z$ conforme $T$ varia, isto é,

$$
s=\operatorname{sign}\left\{\operatorname{Re}\left(\left.\frac{d z}{d T}\right|_{z=i y}\right)\right\} .
$$


Observemos que, como o lado esquerdo de (2.2) é uma função analítica de $z$ e $T$, então uma raiz $z$ simples será uma função diferenciável de $T$.

O primeiro resultado necessário para provarmos o Teorema (2.2) é a seguinte Proposição:

Proposição 2.1. Sejam $P$ e $Q$ duas funções analíticas definidas no semi-plano direito, $\operatorname{Re}(z)>-\delta, \delta>0$ satisfazendo as seguintes condições:

(i) $P(z)$ e $Q(z)$ não possuem zeros imaginários comuns;

(ii) $\overline{P(-i y)}=P(i y)$ e $\overline{Q(-i y)}=Q(i y)$, para $y$ real;

(iii) $P(0)+Q(0) \neq 0$.

Então,

(1) Se iy $(y>0)$ e $T$ satisfazem (2.2), e se iy for uma raiz simples $e$ $s \neq 0$, então y é uma raiz simples de $F(y)=0$ e a raiz $z(T)$ de (2.2) cruza o eixo imaginário (quando $T$ cresce) na direção dada por $s=\operatorname{sign}\left\{F^{\prime}(y)\right\}$;

(2) iy $(y>0)$ é uma raiz simples de (2.3) se, e somente se, y é uma raiz simples de $F(y)=0$. Então para este $y$, existem infinitos valores de $T$ satisfazendo (2.6) e para cada valor de $T, z=i y$ é uma raiz simples de (2.2) e $s \neq 0$.

Vale observar que a hipótese (i) não é uma restrição importante, pois se $z=i y$ for um zero imaginário comum de $P$ e $Q$, então $P(z)+Q(z) e^{-T z}=(i y)^{k}\left(P_{1}(z)+\right.$. $\left.Q_{1}(z) e^{-T z}\right)$, onde $k$ é um inteiro e $P_{1}, Q_{1}$ não possuem zeros imaginários comuns e assim a proposição pode ser aplicada para $P_{1}(z)+Q_{1}(z) e^{-T z}=0$.

A hipótese (iii) equivale a afirmar que $z=0$ não é uma raiz. Se $z=0$ for uma raiz comum de $P$ e $Q$, removemos um fator comum $z^{k}$. Se $P(0)+Q(0)=0$, mas $z=0$ não for uma raiz comum de $P$ e $Q$, então $z=0$ é um zero para todo $T \geq 0 \mathrm{e}$ daí, por definição, a equação não será estável.

\section{Prova:}

Como vimos, é possível, a partir de iy determinarmos valores de $T$ que satisfaçam (2.2). Se $z=i y$, para $y \neq 0$, for uma raiz de (2.2), segue da hipótese (ii) que podemos escolher $y>0$ sem perda de generalidade. E assim, estão satisfeitas (2.5), (2.6).

É claro que $Q_{R}$ e $Q_{I}$ não são ambos nulos, caso contrário teríamos, por (2.3), $Q(i y)=P(i y)=0$, o que contraria a hipótese (i). Provemos a conclusão (1) da 
Proposição.

Sejam iy e $T$ satisfazendo (2.2), e consequentemente satisfazendo (2.3), (2.5) e (2.6). Vamos olhar para a raiz $z=x+i y$ de (2.2) como uma função de $T$, e determinar a direção do movimento de $z$ conforme $T$ cresce, ou seja, vamos determinar

$$
s=\operatorname{sign}\left\{\operatorname{Re}\left(\left.\frac{d z}{d T}\right|_{z=i y}\right)\right\}=\operatorname{sign}\left\{\left.\frac{d}{d T} \operatorname{Re}(z)\right|_{z=i y}\right\} .
$$

Por hipótese, o lado esquerdo de (2.2) é uma função analítica de $z$ e $T$ e como $z=z(T)$, segue do Teorema das Funções Implícitas que $z=z(T)$ é uma função diferenciável com respeito a $T$ em uma vizinhança de uma raiz iy. Derivando ambos os membros de (2.2) em relação a $T$, e supondo que $P$ e $Q$ sejam independentes de $T$, temos

$$
P^{\prime}(z) \frac{d z}{d T}+Q^{\prime}(z) \frac{d z}{d T} e^{-T z}-Q(z)\left(z+T \frac{d z}{d T}\right) e^{-T z}=0
$$

então

$$
\frac{d z}{d T}\left[P^{\prime}(z)+Q^{\prime}(z) e^{-T z}-Q(z) T e^{-T z}\right]=Q(z) z e^{-T z},
$$

consequentemente:

$$
\frac{d z}{d T}=\frac{Q(z) z}{P^{\prime}(z) e^{T z}+Q^{\prime}(z)-T Q(z)}
$$

Para que $\frac{d z}{d t}$ esteja bem definida, devemos ter que $P^{\prime}(z) e^{T z}+Q^{\prime}(z)-T Q(z) \neq 0$, o que de fato ocorre se $z$ for uma raiz simples de (2.2), pois em uma raiz múltipla temos

$$
P^{\prime}(z)+\left[Q^{\prime}(z)-T Q(z)\right] e^{-T z}=0
$$

e de (2.2) podemos substituir $e^{-T z}=\frac{-P(z)}{Q(z)}$ em (2.9) e obter:

$$
P^{\prime}(z) Q(z)-P(z) Q^{\prime}(z)+T P(z) Q(z)=0,
$$

o que implica $P^{\prime}(z)\left[-P(z) e^{T z}\right]-P(z) Q^{\prime}(z)+T P(z) Q(z)=0$, e então 


$$
P^{\prime}(z) e^{T z}+Q^{\prime}(z)-T Q(z)=0 .
$$

Portanto, uma raiz $z$ de (2.2) é uma função diferenciável de $T$, exceto nos pontos onde a raiz é múltipla.

Fazendo $Q(z)=-P(z) e^{T z}$, segue de (2.8) que

$$
\left(\frac{d z}{d T}\right)^{-1}=-\frac{P^{\prime}(z)}{z P(z)}+\frac{Q^{\prime}(z)}{z Q(z)}-\frac{T}{z} .
$$

Vejamos como podemos escrever $s$ de maneira mais simples, usando as expressões acima. Como $\operatorname{sign}\{\operatorname{Re}(w)\}=\operatorname{sign}\left\{\operatorname{Re}\left(w^{-1}\right\}\right.$, para qualquer complexo $w$, temos

$$
\begin{aligned}
s=\operatorname{sign}\left\{\left.\frac{d}{d T} \operatorname{Re}(z)\right|_{z=i y}\right\} & \stackrel{(2.11)}{=} \operatorname{sign}\left\{\operatorname{Re}\left[\frac{-P^{\prime}(i y)}{i y P(i y)}+\frac{Q^{\prime}(i y)}{i y Q(i y)}-\frac{T}{i y}\right]\right\} \\
& =\operatorname{sign}\left\{\operatorname{Re}\left[\frac{P^{\prime}(i y) i}{y P(i y)}-\frac{Q^{\prime}(i y) i}{y Q(i y)}+\frac{T i}{y}\right]\right\} \\
& =\operatorname{sign}\left\{\operatorname{Re}\left[\frac{P^{\prime}(i y) i}{y P(i y)}-\frac{Q^{\prime}(i y) i}{y Q(i y)}\right]\right\} \\
& =-\operatorname{sign}\left\{\operatorname{Im}\left[\frac{P^{\prime}(i y)}{y P(i y)}-\frac{Q^{\prime}(i y)}{y Q(i y)}\right]\right\} .
\end{aligned}
$$

E como $y>0$, segue por (2.3) que:

$$
\frac{P^{\prime}(i y)}{y P(i y)}-\frac{Q^{\prime}(i y)}{y Q(i y)}=\frac{-Q^{\prime}(i y) \overline{Q(i y)}+P^{\prime}(i y) \overline{P(i y)}}{y|P(i y)|^{2}},
$$

como $\left(y|P(i y)|^{2}\right)>0$, temos que:

$$
s=-\operatorname{sign}\left\{\operatorname{Im}\left[P^{\prime}(i y) \overline{P(i y)}-Q^{\prime}(i y) \overline{Q(i y)}\right]\right\} .
$$

Usando (2.4), podemos melhorar (2.13). Usando também que

$$
P^{\prime}(i y)=\frac{1}{i} \frac{d}{d y} P(i y)=-i \frac{d}{d y}\left(P_{R}(y)+i P_{I}(y)\right)=-P_{R}^{\prime}(y) i+P_{I}^{\prime}(y),
$$

e escrevendo $\frac{d P_{R}}{d y}(y)=P_{R}^{\prime}(y)$ e o mesmo para $Q$, temos

$$
\begin{aligned}
& -\operatorname{Im}\left[P^{\prime}(i y) \overline{P(i y)}-Q^{\prime}(i y) \overline{Q(i y)}\right] \\
= & -\operatorname{Im}\left[\left(P^{\prime}{ }_{I}(y)-i P^{\prime}{ }_{R}(y)\right)\left(P_{R}(y)-i P_{I}(y)\right)-\left(Q^{\prime}{ }_{I}(y)-i Q_{R}^{\prime}(y)\right)\left(Q_{R}(y)-i Q_{I}(y)\right]\right.
\end{aligned}
$$


e assim,

$$
-\operatorname{Im}\left[P^{\prime}(i y) \overline{P(i y)}-Q^{\prime}(i y) \overline{Q(i y)}\right]=P_{R} P_{R}^{\prime}+P_{I} P_{I}^{\prime}-Q_{R} Q_{R}^{\prime}-Q_{I} Q_{I}^{\prime} .
$$

Além disso, podemos escrever

$$
F(y)=|P(i y)|^{2}-|Q(i y)|^{2}=P_{R}^{2}(y)+P_{I}^{2}(y)-Q_{R}^{2}(y)-Q_{I}^{2}(y) .
$$

O observemos que, se $i y$ for uma raiz de (2.2), então $y$ é um zero simples de $F(y)=0$ se, e somente se, $y$ é uma raiz simples de (2.3). Derivando $F(y)$, temos

$$
F^{\prime}(y)=2\left(P_{R} P_{R}^{\prime}+P_{I} P_{I}^{\prime}-Q_{R} Q_{R}^{\prime}-Q_{I} Q_{I}^{\prime}\right) .
$$

Combinando as três últimas equações (2.13), (2.14) e (2.15), obtemos:

$$
\begin{aligned}
\operatorname{sign}\left[F^{\prime}(y)\right] & =\operatorname{sign}\left[2\left(P_{R} P^{\prime}{ }_{R}+P_{I} P_{I}^{\prime}-Q_{R} Q^{\prime}{ }_{R}-Q_{I} Q_{I}^{\prime}\right]\right. \\
& =\operatorname{sign}\left[P_{R} P_{R}^{\prime}+P_{I} P_{I}^{\prime}-Q_{R} Q_{R}^{\prime}-Q_{I} Q_{I}^{\prime}\right] \\
& =\operatorname{sign}\left[-\operatorname{Im}\left[P^{\prime}(i y) \overline{P(i y)}-Q^{\prime}(i y) \overline{Q(i y)}\right]\right] \\
& =-\operatorname{sign}\left[\operatorname{Im}\left[P^{\prime}(i y) \overline{P(i y)}-Q^{\prime}(i y) \overline{Q(i y)}\right]\right]
\end{aligned}
$$

portanto, segue de (2.13) que

$$
s=\operatorname{sign}\left\{F^{\prime}(y)\right\} .
$$

Assim, provamos que, em qualquer raiz simples $i y$, com $y>0$, onde ocorre o cruzamento no eixo imaginário com velocidade $s \neq 0$, a direção do cruzamento é dada por (2.13) ou (2.17).

Provemos (2). Suponhamos que $y$ seja uma raiz simples de $F(y)=0$, com $y>0$ então $F^{\prime}(y) \neq 0$, e como já foi visto segue que $y$ é uma raiz simples de (2.3). Sabendo que vale (i) podemos supor $Q(i y) \neq 0$ e assim é possível determinar valores de $T$ tal que (2.6) esteja satisfeita. Portanto, pela parte anterior, temos que para cada valor de $T$, uma raiz $z(T)$ cruza o eixo imaginário em iy na direção dada em (2.17). $\mathrm{E}$ desde que $s$ é dado por (2.12), temos que

$$
\frac{P^{\prime}(i y)}{y P(i y)}-\frac{Q^{\prime}(i y)}{y Q(i y)}-\frac{T}{i y} \neq 0
$$

e daí,

$$
P^{\prime}(i y) Q(i y)-P(i y) Q^{\prime}(i y)+T P(i y) Q(i y) \neq 0
$$


o que implica que (2.10) falha, consequentemente $i y$ é uma raiz simples de (2.2), o que conclue 2) e completa a demonstração.

Vamos agora estudar a equação (2.2) no caso em que $P$ e $Q$ são funções polinomiais. Tal caso merece um destaque pois aparece com freqüência na literatura.

Proposição 2.2. Sejam $P$ e $Q$ funções polinomiais com coeficientes reais não tendo raízes comuns sobre o eixo imaginário. Suponhamos que o grau de $P$, denotado por $n$, seja maior que o grau de $Q$. Sejam iy $y_{k}$ raízes simples de (2.2), $k \leq n$, com $y_{k}>0$, e $r_{k}=y_{k}^{2}$. Então a direção de cruzamento $s=s_{k}$ em ì $y_{k}$ é dada por

$$
s_{k}=\operatorname{sign}\left\{\prod_{\substack{j=1 \\ j \neq k}}^{n}\left(r_{k}-r_{j}\right)\right\}
$$

\section{Prova:}

É claro que $P_{R}^{2}, P_{I}^{2}, Q_{R}^{2}$ e $Q_{I}^{2}$ são polinômios que contêm potências pares de $y$, e como o grau de $P$ é maior que o grau de $Q$, segue que $F(y)$ é um polinômio de grau $2 n$ em $y$, isto é, um polinômio de grau $n$ em $y^{2}$. Se $x=y^{2}$, com $y>0$ e se $F(y)=\hat{F}(x)$, então $\hat{F}(x)$ é um polinômio de grau $n$, que pode ser escrito na forma fatorada

$$
\hat{F}(x)=\prod_{j=1}^{n}\left(x-r_{j}\right)
$$

Desde que $F^{\prime}(y)=2 y \frac{d \hat{F}}{d x}(x)$, segue de (2.17), que em qualquer raiz simples $i y_{k}$ de (2.2) $\operatorname{com} y_{k}>0$,

$$
s=\operatorname{sign}\left\{\left.\left(\frac{d \hat{F}}{d x}\right)\right|_{x=y_{k}^{2}}\right\}=\operatorname{sign}\left\{\sum_{i=1}^{n}\left(\prod_{j \neq i}\left(x-r_{j}\right)\right)_{x=y_{k}^{2}}\right\} .
$$

Isto é fácil de ver usando indução finita e a regra de derivação do produto. 
Vamos utilizar agora a equação (2.20). Se temos $x=r_{k}$, para $k$ fixo entre 1 e $n$, segue (2.18), pois, para $x=r_{k}$

$$
\begin{gathered}
\sum_{i=1}^{n}\left(\prod_{j \neq i}\left(x-r_{j}\right)\right)=\left(r_{k}-r_{2}\right) \ldots\left(r_{k}-r_{k}\right) \ldots\left(r_{k}-r_{n}\right)+\left(r_{k}-r_{1}\right) \ldots\left(r_{k}-r_{k}\right) \ldots\left(r_{k}-r_{n}\right)+ \\
+\ldots+\left(r_{k}-r_{1}\right) \ldots\left(r_{k}-r_{k-1}\right)\left(r_{k}-r_{k+1}\right) \ldots\left(r_{k}-r_{n}\right) \\
\left.+\ldots+\left(r_{k}-r_{1}\right) \ldots\left(r_{k}-r_{k}\right) \ldots\left(r_{k}-r_{n}\right)=\prod_{\substack{j=1 \\
j \neq k}}^{n}\left(r_{k}-r_{j}\right)\right)
\end{gathered}
$$

logo,

$$
s_{k}=\operatorname{sign}\left\{\sum_{i=1}^{n}\left(\prod_{\substack{j=1 \\ j \neq k}}^{n}\left(r_{k}-r_{j}\right)\right)\right\}, \text { o que conclue a prova. }
$$

Note que a equação (2.18) nos fornece uma maneira simples para determinarmos a estabilidade de soluções de uma dada equação, que consiste em resolver $F(y)=0$, encontrando as possíveis raízes $i y_{k}$, nas quais raízes de (2.2) cruzam o eixo imaginário. Se $F(y)=0$ não possuir raízes positivas, então nenhum cruzamento ocorre e conclui-se que a equação é estável para todo $T \geq 0$ ou é instável para todo $T \geq 0$.

No caso em que $F(y)=0$ possuir pelo menos uma raiz positiva, temos o seguinte resultado, cuja prova é conseqüência imediata da Proposição (2.2).

Corolário 2.1. Suponhamos que as raízes positivas $r_{1}, r_{2}, \ldots, r_{p}$ de $\hat{F}(x)=0$ sejam distintas e $r_{1}>r_{2}>\ldots>r_{p}>0(p \leq n)$. Então $\pm i y_{k}= \pm \sqrt{r_{k}},(k=1,2, \ldots, p)$ são as possiveis raizes de (2.2) sobre o eixo imaginário. Se essas raizes forem simples, entẫo a direção do cruzamento $s_{k}$ em iy $y_{k}$ é dada por

$$
s_{k}=\operatorname{sign}\left\{\prod_{\substack{j=1 \\ j \neq k}}^{p}\left(r_{k}-r_{j}\right)\right\} .
$$

Observação 2.1. Em vista do Corolário (2.1) podemos afirmar que a direção do cruzamento na maior raiz $r_{1}$ é sempre da esquerda para a direita, pois

$$
s_{1}=\operatorname{sign}\left\{\prod_{j=2}^{p}\left(r_{1}-r_{j}\right)\right\}=\left(r_{1}-r_{2}\right) \ldots\left(r_{1}-r_{p}\right)>0 .
$$


A direção do cruzamento em $r_{2}$ é da direita para a esquerda, pois

$$
s_{2}=\operatorname{sign}\left\{\prod_{\substack{j=1 \\ j \neq 2}}^{p}\left(r_{2}-r_{j}\right)\right\}=\left(r_{2}-r_{1}\right) \ldots\left(r_{2}-r_{p}\right)<0 .
$$

E assim sucessivamente.

Se acaso existir uma única raiz positiva $r_{1}$, foi visto que a direção do cruzamento sempre é para a direita. Se existirem duas raízes positivas $r_{1}$ e $r_{2}$, os cruzamentos são à direita na primeira e à esquerda na segunda.

2) Para cada raiz positiva $r_{k}$ de $\hat{F}(x)$ existe uma seqüência infinita de valores $T=T_{k, m}$ com $m=1,2 \cdots$, nos quais as raizes de (2.2) cruzam o eixo imaginário. De fato, para cada $r_{k}$ solução de $\hat{F}(x)=0$, temos $y_{k}^{2}=r_{k}$, o que implica $y_{k}=\sqrt{r_{k}}>0$, tal que iy $y_{k}$ é solução de (2.3) e portanto satisfaz, como vimos, (2.6). Como o seno e o cosseno são funções periódicas, é possível construir uma seqüência $\left\{T_{k, m}\right\}$ que sempre satisfaça:

$$
\operatorname{sen}\left(T_{k, m} y_{k}\right)=\operatorname{sen}\left(T y_{k}\right), \quad \cos \left(T_{k, m} y_{k}\right)=\cos \left(T y_{k}\right),
$$

para qualquer $m$ natural.

Se chamarmos $\theta_{k}=T y_{k}$ solução de (2.6), então $\theta_{k}+2 \pi$ também é solução. Logo, $y_{k} T_{k, 1}=\theta_{k_{1}}+2 \pi$, implica

$$
T_{k, 1}=\frac{\theta_{k_{1}}}{y_{k}}+\frac{2 \pi}{y_{k}}, \quad T_{k, 2}=\frac{\theta_{k_{2}}}{y_{k}}+\frac{4 \pi}{y_{k}} .
$$

$e$ assim por diante. Então, para um $k$ fixo, raízes cruzam em 土iy $k$, nos valores $\left\{T_{k, m}\right\}$ com $m=1,2, \cdots$, e satisfazem

$$
T_{k, m+1}-T_{k, m}=\frac{2 \pi}{y_{k}}=\frac{2 \pi}{\sqrt{r_{k}}}
$$

Como, por hipótese as raízes são simples, exatamente um par de raízes, $\left\{i y_{k},-i y_{k}\right\}$ cruzam em cada $T_{k, m}$.

Exemplificando a observação acima, suponhamos que existam duas raízes positivas, $r_{1}$ e $r_{2}$, com $r_{1}>r_{2}>0$. Então os cruzamentos do lado esquerdo para o direito ocorrem no ponto $i y_{1}$ sobre o eixo imaginário, nos tempos, $T_{1,1}, T_{1,1}+2 \pi / y_{1}, T_{1,1}+$ $4 \pi / y_{1}, \cdots$. E os cruzamentos do lado direito para o esquerdo ocorrem em $i y_{2}$ sobre o eixo imaginário, nos tempos $T_{2,1}, T_{2,1}+2 \pi / y_{2}, T_{2,1}+4 \pi / y_{2}, \cdots$. Como $y_{1}>y_{2}$, temos 
$2 \pi / y_{1}<2 \pi / y_{2}$. Portanto, cruzamentos para a direita ocorrem com mais freqüência que cruzamentos para a esquerda.

A seguir, examinaremos como a estabilidade de certos modelos muda conforme o retardo $T$ cresce, a fim de apresentarmos um resultado geral sobre certas equações, que apresentam a equação característica do tipo (2.2).

O lema abaixo traz uma análise de estabilidade para uma equação diferencial funcional de primeira ordem e linear.

Lema 2.1. Considere a equação:

$$
\dot{x}(t)=a x(t)+b x(t-T),
$$

com $T>0$. Se a equação acima for estável para $T=0$ então ou ela é estável para todo $T \geq 0$, ou existe um valor $T^{*}$ tal que ela é estável para $T<T^{*}$ e instável para $T>T^{*}$.

\section{Prova:}

Sabemos que a estabilidade da solução nula depende das raízes da equação característica associada:

$$
z-a-b e^{-T z}=0 .
$$

Lembremos que, para uma equação diferencial com retardamento, o supremo das partes reais das raízes da equação característica varia continuamente em $T$, veja [11]. Assim, se existir uma troca de estabilidade quando $T$ varia, ela corresponde a uma raiz que deve ser imaginária pura $z=i y$. Para a equação (2.24) qualquer raiz imaginária pura deve ser simples, pois se a raiz $z$ for múltipla temos,

$$
\begin{gathered}
z-a-b e^{-T z}=0 \\
1+b T \cdot e^{-T z}=0
\end{gathered}
$$

o que implica $b e^{-T z}=\frac{-1}{T}$, e então $z=a-\frac{1}{T}$, que é um número real, o que nos leva a uma contradição.

Como $z-a-b e^{-T z}$ é uma função analítica de $z$ e $T$, segue que a raiz $z(T)$ é uma função diferenciável de $T$ em uma vizinhança de uma raiz iy. Derivando ambos os membros de (2.24) com respeito a $T$, obtemos

$$
\frac{d z}{d T}+z b e^{-T z}+T b e^{-T z} \frac{d z}{d T}=0
$$


o que implica

$$
\frac{d z}{d T}=\frac{-z b e^{-T z}}{1+T b e^{-T z}}
$$

Em valores de $T$, tal que $z=i y$, temos,

$$
\frac{d z}{d T}=\frac{-y b i e^{-i T y}}{1+T b e^{-i T y}}=\frac{-i y b(\cos (T y)-i \operatorname{sen}(T y))-i b^{2} T y^{2}}{\left|1+T b e^{-T i y}\right|}
$$

Sabendo que $\frac{d}{d T} R e(z)=R e\left(\frac{d z}{d T}\right)$, segue que

$$
R e\left(\frac{d z}{d T}\right)=\frac{-b y \operatorname{sen}(T y)}{[1+b T \cos (T y)]^{2}+[b T \operatorname{sen}(T y)]^{2}}
$$

Por outro lado, em $z=i y$, devemos ter de (2.24) que

$$
i y-a-b e^{-i y T}=0,
$$

portanto, $i y-a-b\left(\cos (T y)-i \operatorname{sen}(T y)=0\right.$ implica $-y=b \operatorname{sen}(-T y) \operatorname{eos}(T y)=\frac{-a}{b}$ e assim

$$
\frac{d}{d T} R e(z)=\frac{y^{2}}{(1-a T)^{2}+(y T)^{2}} .
$$

Podemos afirmar o seguinte:

1) se $y \neq 0,(2.25)$ é maior que zero, e então a raiz deve passar do semi-plano negativo para o positivo, conforme $T$ cresce.

2) $y=0$, corresponde à raiz nula $z=0$, o que é impossível, pois se isso ocorrer, teremos por (2.24), que $-(a+b)=0$, o que contraria a hipótese $a+b \neq 0$.

Portanto, raízes só podem cruzar o eixo imaginário do lado esquerdo para o direito, conforme $T$ cresce. Assim, se a equação for estável em $T=0$, a estabilidade é perdida em algum ponto crítico, $T^{*}$, de $T$ e nunca é recuperada.

Lema 2.2. Seja $f(z, T)=z^{2}+a z+c+(b z+d) e^{-T z}$, onde $a, b, c, d, T$ são números reais e $T \geq 0$. Então, conforme $T$ varia, a soma das multiplicidades de zeros de $f$ no semi-plano aberto à direita pode mudar somente se um zero aparece ou se um zero cruza o eixo imaginário. 


\section{Prova:}

Seja $z=z(T)$ uma raiz qualquer de $f(z, T)=0$. Consideremos $r>0$ arbitrariamente pequeno, e o disco de raio $r$ ao redor de $z(T)$. Então, segue do Teorema de Rouché que, para $T^{\prime}$ suficientemente próximo de $T$, a multiplicidade total das raízes no disco é igual a multiplicidade de $z(T)$. Assim, uma raiz $z(T)$ não pode repentinamente desaparecer ou aparecer ou mudar sua multiplicidade em um ponto finito do plano.

Seja $M(T)$ a multiplicidade total (finita) de zeros no semi-plano direito aberto. Suponhamos, por absurdo, que $M(T)$ muda, e que raízes não aparecem e nem cruzam o eixo imaginário. Logo, isto só poderá ocorrer se existir um $T^{*}$ e uma raiz $z(T)$ tal que:

$$
|z(T)| \longrightarrow \infty, \quad T \longrightarrow T^{*},
$$

$\operatorname{com} \operatorname{Re}(z(T)) \geq 0$. Escrevendo,

$\frac{f(z, T)}{z^{2}}=1+a z^{-1}+b z^{-1} e^{-T z}+c z^{-2}+d z^{-2} e^{-T z}=1+z^{-2}\left(a z+b z e^{-T z}+c+d e^{-T z}\right)$

e sendo $\left|e^{-T z(T)}\right| \leq 1$, segue que

$$
\frac{f(z, T)}{z^{2}} \rightarrow 1, \quad \text { quando } \quad|z(T)| \rightarrow \infty
$$

e isto contradiz o fato de que $f(z(T), T)=0$.

Consideremos agora, a seguinte equação geral de segunda ordem, com atrito retardado e força restauradora retardada, com o mesmo retardo:

$$
\frac{d^{2} x}{d t^{2}}+a \frac{d x}{d t}+b \frac{d x}{d t}(t-T)+c x(t)+d x(t-T)=0
$$

onde $a+b \neq 0, c+d \neq 0$.

Esta equação pode surgir de um sistema não linear com um retardo e pode ser encontrada em [4].

Para realizarmos uma análise desta equação, vamos considerar alguns dos parâmetros acima nulos, resultando em algumas equações com importância em várias aplicações 
físicas.

\section{Primeira Equação}

Fazendo $d=0$, temos a seguinte equação retardada de segunda ordem que envolve a força de atrito:

$$
\frac{d^{2} x}{d t^{2}}+a \frac{d x}{d t}+b \frac{d x}{d t}(t-T)+c x(t)=0
$$

onde $a, b$ são constantes não negativas e $c>0, a+b>0$ e $T>0$. [4] mostra que esta equação sugere um modelo para pequenas vibrações de um pêndulo de massa um, onde $x$ é o deslocamento a partir da posição equilíbrio, $c x(t)$ é a força linear restauradora, $a \dot{x}(t)$ é a força natural de atrito e $b \dot{x}(t-T)$ é a força de atrito introduzida com um retardo $T$. Mostremos que, para $T$ crescendo de 0 a $\infty$, a solução nula da equação é alternadamente estável, instável e estável novamente, com um número finito de trocas de estabilidade e com a instabilidade se instalando para $T$ suficientemente grande. Durante a análise desta equação, utilizaremos o Lema (2.2).

A equação característica associada é:

$$
z^{2}+a z+c+b z e^{-T z}=0,
$$

onde $P(z)=z^{2}+a z+c$ e $Q(z)=b z$, assim grau de $P$ é dois e o grau de $Q$ é um.

Seja $z=i y$ uma raiz imaginária pura, com $y>0$, pois raízes complexas ocorrem em pares conjugados. Supondo $c>0$, temos que $z=0$ não pode ser uma raiz de (2.28). Substituindo iy na equação característica, obtemos

$$
-y^{2}+c+b y \operatorname{sen}(T y)+i(a y+b y \cos (T y))=0,
$$

igualando partes real e imaginária, temos

$$
\left\{\begin{array}{l}
y^{2}-c-b y \operatorname{sen}(T y)=0 \\
y(a+b \cos (T y))=0 .
\end{array}\right.
$$

Consideremos alguns casos:

$$
\text { Primeiro Caso) } \quad a>b \geq 0 \text {. }
$$

Como $y \neq 0$, vale, por (2.29) que

$$
|\cos (T y)|=\left|-\frac{a}{b}\right|>1
$$


e portanto não existe solução real $y$ de (2.28). Assim, (2.28) não possue raízes imaginárias. Pelo Lema (2.2) a multiplicidade total das raízes no semi-plano direito não muda. Vejamos se a equação pode ser estável em $T=0$.

Para $T=0$, temos uma equação quadrática, $z^{2}+(a+b) z+c=0$. Fazendo $z=x+i y$ uma solução desta equação e igualando partes real e imaginária, ficamos com:

$$
\begin{cases}x^{2}-y^{2}+(a+b) x+c & =0 \\ 2 x y+a y+b y & =0 .\end{cases}
$$

Da última igualdade temos duas possibilidades: $y=0$ ou $x=-\frac{(a+b)}{2}<0$. Se $y=0$, da primeira igualdade, temos $x^{2}+(a+b) x+c=0$. Como $a+b>0$ e $c>0$, segue que $x<0$. Logo, $\operatorname{Re}(z)<0$, e portanto todas as raízes estão no semi-plano esquerdo, independentemente de T. Assim, a solução nula da equação (2.27) é estável para todo $T \geq 0$.

Segundo Caso) $\quad 0 \leq a<b$.

$\mathrm{Da}$ equação (2.29) segue que

$$
\cos (T y)=-\frac{a}{b}<0
$$

e portanto

$$
T y=\cos ^{-1}\left(\frac{-a}{b}\right)+2 n \pi, \quad n=0,1,2, \cdots .
$$

Definimos:

$$
\begin{array}{ll}
\theta_{1}=\cos ^{-1}\left(\frac{-a}{b}\right), & \frac{\pi}{2} \leq \theta_{1}<\pi, \\
\theta_{2}=\cos ^{-1}\left(\frac{-a}{b}\right), & \pi<\theta_{2}<\frac{3 \pi}{2} .
\end{array}
$$

Na escolha de $\theta_{1}$, substituindo $T y$ no sen $(T y)$, temos:

$$
\begin{aligned}
& \operatorname{sen}(T y)=\operatorname{sen}\left(\cos ^{-1}\left(\frac{-a}{b}\right)+2 n \pi\right) \\
& \quad=\operatorname{sen}\left(\theta_{1}+2 n \pi\right)=\sqrt{1-\cos ^{2}\left(\theta_{1}+2 n \pi\right)}=\sqrt{1-\frac{a^{2}}{b^{2}}} .
\end{aligned}
$$


Substituindo em 2.29, obtemos:

$$
y^{2}-c-b y \sqrt{1-\frac{a^{2}}{b^{2}}}=0
$$

como $b>0$, temos

$$
y^{2}-y \sqrt{b^{2}-a^{2}}-c=0
$$

As raízes são dadas por:

$$
y=\frac{\sqrt{b^{2}-a^{2}} \pm \sqrt{b^{2}-a^{2}+4 c}}{2}
$$

como $c>0$, temos uma única raiz positiva

$$
y_{1}=\frac{\sqrt{b^{2}-a^{2}}+\sqrt{b^{2}-a^{2}+4 c}}{2} .
$$

$\mathrm{Na}$ escolha de $\theta_{2}$, podemos repetir os mesmos argumentos, pois o que muda é o sinal do $\operatorname{sen}(T y)$, e ficamos com

$$
y^{2}+y \sqrt{b^{2}-a^{2}}-c=0 .
$$

As raízes são dadas por:

$$
y=\frac{-\sqrt{b^{2}-a^{2}} \pm \sqrt{b^{2}-a^{2}+4 c}}{2}
$$

como $c>0$, temos uma única raiz positiva

$$
y_{2}=\frac{-\sqrt{b^{2}-a^{2}}+\sqrt{b^{2}-a^{2}+4 c}}{2} .
$$

Assim, temos duas seqüências de valores positivos de $T$ correspondendo às duas raízes positivas e imaginárias puras $y_{1}$ e $y_{2}$ :

$$
T_{n, 1}=\frac{\theta_{1}+2 n \pi}{y_{1}}, \quad T_{n, 2}=\frac{\theta_{2}+2 n \pi}{y_{2}}, \quad(n=0,1,2, \cdots),
$$

respectivamente. Mostremos que, cada uma dessas raízes é simples. De fato, derivando ambos os membros de (2.28), em relação a $z$, obtemos $2 z+a+(b-b T z) e^{-T z}=0$ e dessa equação podemos tirar que

$$
e^{-T z}=\frac{-2 z-a}{b-b T z}=-\frac{2 z}{b(1-T z)}-\frac{a}{b(1-T z)} .
$$


Resolvendo (2.28) para $z \neq 0$ e substituindo o valor de $e^{-T z}$, segue que

$$
z^{2}+a z+c+b z\left(-\frac{2 z}{b(1-T z)}-\frac{a}{b(1-T z)}\right)=0
$$

o que implica

$$
\begin{gathered}
z^{2}(1-T z)+a z(1-T z)-2 z^{2}-z a+c(1-T z)=0, \\
T z^{3}+(a T+1) z^{2}+c T z-c=0 .
\end{gathered}
$$

Para $z=i y$, devemos ter

$$
-T y^{3} i-(a T+1) y^{2}+c T i y-c=0,
$$

igualando partes real e imaginária, ficamos na parte real com

$$
y^{2}(a T+1)+c=0, \quad 0<y^{2}=-\frac{c}{a T+1}<0,
$$

o que é absurdo. Concluímos que qualquer raiz sobre o eixo imaginário deve ser simples.

Continuando nossa análise, vamos derivar ambos os membros de (2.28) com respeito a $T$,

$$
2 z \frac{d z}{d T}+a \frac{d z}{d T}+b\left(\frac{d z}{d T} e^{-T z}-z\left(z+T \frac{d z}{d T}\right) e^{-T z}\right)=0
$$

logo,

$$
\frac{d z}{d T}=\frac{b z^{2}}{(2 z+a) e^{T z}+b-b T z}
$$

Para $z=i y$, obtemos

$$
\begin{aligned}
& \frac{d z}{d T}=\frac{-b y^{2}}{(2 y i+a) e^{T i y}+b-b T y i} \\
& =\frac{-b y^{2}[-2 y \operatorname{sen}(T y)+a \cos (T y)+b-(2 y \cos (T y)+a \operatorname{sen}(T y)-b T y) i]}{(-2 y \operatorname{sen}(T y)+a \cos (T y)+b)^{2}+(2 y \cos (T y)+a \operatorname{sen}(T y)-b T y)^{2}} .
\end{aligned}
$$


Como $\frac{d}{d T} \operatorname{Re}(z)=\operatorname{Re}\left(\frac{d z}{d T}\right)$, temos

$$
\frac{d}{d T} R e(z)=-b y^{2}[a \cos (T y)-2 y \operatorname{sen}(T y)+b] D^{-1}
$$

onde $D=(-2 y \operatorname{sen}(T y)+a \cos (T y)+b)^{2}+(2 y \cos (T y)+a \operatorname{sen}(T y)-b T y)^{2}>0$ então

Quando $T=T_{n, 1}$ e $y=y_{1}$, temos que $\cos (T y)=\frac{-a}{b}$ e $\operatorname{sen}(T y)=\sqrt{1-\frac{a^{2}}{b^{2}}}$ e

$$
\frac{d}{d T} R e(z)=-y_{1}^{2}\left[b^{2}-a^{2}-2 y_{1} \sqrt{b^{2}-a^{2}}\right] D^{-1}>0
$$

concluímos que todas as raízes que cruzam o eixo imaginário em $i y_{1}$ devem cruzar do lado esquerdo para o direito, conforme $T$ cresce, passando pelos valores $T_{n, 1}$.

Por outro lado, quando $T=T_{n, 2}$ e $y=y_{2}$, temos

$$
\cos (T y)=\frac{-a}{b}, \quad \operatorname{sen}(T y)=-\sqrt{1-\frac{a^{2}}{b^{2}}}
$$

e então,

$$
\frac{d}{d T} R e(z)=-y_{2}^{2}\left[b^{2}-a^{2}+2 y_{2} \sqrt{b^{2}-a^{2}}\right] D^{-1}<0
$$

assim, concluímos que todas as raízes que cruzam o eixo imaginário em $i y_{2}$ devern cruzar do lado direito para o esquerdo, conforme $T$ cresce, passando pelos valores $T_{n, 2}$.

Para darmos prosseguimento à nossa análise é preciso provar a seguinte proposição.

Proposição 2.3. Para quaisquer $a, b$ e $c$ fixos com $a<b$, existe um inteiro $k$ tal que

$$
T_{0,1}<T_{0,2}<T_{1,1}<T_{1,2}<\ldots<T_{k-1,1}<T_{k-1,2}<T_{k, 1}
$$


e consequentemente, quando $0 \leq T<T_{k, 1}$, temos alternadamente $k$ trocas de estabilidade e além disso, o sistema é instável para todo $T>T_{k, 1}$.

\section{Prova:}

Para demonstrarmos este resultado vamos examinar as condições sob as quais as duas seqüências $\left\{T_{n, 1}\right\}$ e $\left\{T_{n, 2}\right\}$ se alternam. Sabemos que

$$
T_{n, 1}=\frac{\theta_{1}+2 n \pi}{y_{1}}, \quad T_{n, 2}=\frac{\theta_{2}+2 n \pi}{y_{2}} \quad n=1,2, \cdots,
$$

$\operatorname{com} y_{1}>y_{2}$ e $\theta_{1}<\theta_{2}$, logo

$$
T_{0,1}=\frac{\theta_{1}}{y_{1}}<\frac{\theta_{2}}{y_{2}}=T_{0,2}
$$

e também

$$
\left\{T_{n+1,1}\right\}-\left\{T_{n, 1}\right\}=\frac{2 \pi}{y_{1}}<\frac{2 \pi}{y_{2}}=\left\{T_{n+1,2}\right\}-\left\{T_{n, 2}\right\}
$$

Podemos resumir as informações acima da seguinte forma:1) o primeiro cruzamento ocorre em $T=T_{0,1}$, da esquerda para a direita em $i y_{1}$, implicando em instabilidade; 2) o intervalo de tempo entre dois elementos consecutivos da seqüência $\left\{T_{n, 1}\right\}$ é menor que o intervalo de tempo entre dois elementos da seqüência $\left\{T_{n, 2}\right\}$. Logo, deve existir um $k$ inteiro, tal que

$$
T_{k-1,1}<T_{k-1,2}<T_{k, 1}<T_{k+1,1}<T_{k, 2}
$$

Pelo Lema (2.2), a multiplicidade total das raízes no semi-plano direito só pode mudar quando uma raiz cruza o eixo imaginário. Note que, para $0 \leq T<T_{0,1}$, a multiplicidade total no semi-plano direito é zero, pois para $T=0$, estamos supondo que a equação seja estável, e neste intervalo de tempo, nenhuma raiz ainda cruzou o eixo, já que o primeiro cruzamento se dá em $T=T_{0,1}$, e então para $T$ variando neste intervalo temos estabilidade. Agora, para $T_{0,1}<T<T_{0,2}$, a multiplicidade total das raízes no semi-plano direito é exatamente dois, já que, quando $T=T_{0,1}$, duas raízes cruzaram o eixo imaginário do lado esquerdo para o direito, assim, para $T$ variando neste intervalo temos instabilidade. Para $T_{0,2}<T<T_{1,1}$, novamente a multiplicidade total das raízes no semi-plano direito é zero, já que, em $T_{0,2}$ duas raízes cruzaram o eixo imaginário do lado direito para o esquerdo, assim pelo Lema (2.2) a multiplicidade deve mudar, neste caso, deve diminuir para zero e portanto, 
novamente temos estabilidade. Seguindo o mesmo raciocínio, é claro que até o instante $T_{k, 1}$ temos $k$ trocas de estabilidade.

Vejamos que, para $T>T_{k, 1}$ a equação é instável. De fato, para $T_{k, 1}<T<T_{k, 2}$ temos que, pelo menos quatro rázes cruzaram o eixo imaginário, do lado esquerdo para o direito; para $T_{k, 2}<T<T_{k+2,1}$, pelo menos duas raízes cruzaram o eixo imaginário, do lado direito para o esquerdo, e daí a multiplicidade total das raízes no semi-plano direito é pelo menos dois. Portanto, conforme $T$ varia, a multiplicidade das raízes no semi-plano direito é pelo menos dois, o que implica instabilidade.

Para finalizar a análise deste caso, podemos concluir, usando a Proposição (2.3), que existem finitas trocas de estabilidade e que para $T$ suficientemente grande a instabilidade prevalece.

Observação 2.2. No caso, onde $a=b>0$, de (2.29) ficamos com

$$
\begin{gathered}
y^{2}-c-\operatorname{aysen}(T y)=0, \\
a+a \cos (T y)=0 \Rightarrow \cos (T y)=-1 .
\end{gathered}
$$

Assim, se $\operatorname{sen}(T y)=0$, temos $y^{2}=c$, e a única raiz positiva é $y=\sqrt{c}$ e os valores críticos de $T$ são

$$
T_{n}=\frac{(2 n+1) \pi}{\sqrt{c}},
$$

com $n=0,1,2, \cdots$. Neste caso, temos que $\operatorname{Re}\left(\frac{d z}{d T}\right)=0$ nos valores críticos $z=i y$. De fato, (2.32) implica que

$$
\frac{d}{d T}(\operatorname{Re}(z))=-a y^{2}(a \cos (T y)-2 y \operatorname{sen}(T y)+b) D^{-1}=-a y^{2}(-a+a) D^{-1}=0 .
$$

Quando nos deparamos com tal situação, é necessário calcular as derivadas de ordem superiores para determinar se existem raízes que cruzam o eixo. Não realizaremos este cálculo aqui desde que, $s=0$ não se encaixa nas hipóteses da Proposição (2.1). Assim, pode surgir um questionamento no caso de $s=0$, ou mesmo quando a raiz imaginária pura não for simples. Nestes casos, [13] apresenta um critério para resolver as derivadas superiores.

Podemos atribuir uma interpretação física aos resultados acima. Primeiro, se $a>b$, o maior amortecimento não é retardado, e os resultados acima mostram que 
a solução nula permanece estável, qualquer que seja o retardo na parte retardada do amortecimento. Por outro lado, se $a<b$, então o amortecimento retardado é maior que o amortecimento não retardado. Logo, existem intervalos de valores de $T$ para os quais a solução nula é estável e intervalos para os quais é instável. Ocorre instabilidade quando o retardo é de um tamanho que causa uma mudança de fase. Quanto maior é a força de restauração $c$, maior é o número de mudanças de fase, permitindo estabilidade, mas em todos os casos existe instabilidade para $T$ suficientemente grande.

\section{Terceiro Caso)}

Neste caso suponhamos que a solução nula seja instável em $T=0$, o que implica $a+b<0$ ou $c<0$, devido à análise realizada em (2.30).

Vamos verificar sob quais condições, trocas de estabilidade ocorrem quando $T$ cresce. Para isso, vamos analisar algumas situações:

1) Consideremos $a+b<0$ e $c>0$.

Segue que

$$
\cos (T y)=\frac{-a}{b},
$$

esta igualdade vale se $|-a / b|<1$, ou seja, a equação $z^{2}+a z+b e^{-T z}+c=0$ não possue raiz imaginária, a menos que $|a|<|b|$, e como $a+b<0$, devemos ter que $b<0$, para garantir a existência de solução. Vejamos alguns subcasos:

(a) Seja $a>0$.

De $\cos (T y)=-\frac{a}{b}>0$, temos $\theta_{1}=\cos ^{-1}\left(-\frac{a}{b}\right)$, com $0<\theta_{1}<\frac{\pi}{2}$ e $\theta_{2}=$ $\cos ^{-1}\left(-\frac{a}{b}\right), \operatorname{com} \frac{3 \pi}{2}<\theta_{2}<2 \pi$.

$\mathrm{Na}$ escolha de $\theta_{1}$,

$$
\operatorname{sen}(T y)=\sqrt{1-(a / b)^{2}}>0 .
$$

Substituindo em (2.29) e como $b<0$, temos $y^{2}+y \sqrt{b^{2}-a^{2}}-c=0$, e como $c>0$, existe uma única raiz positiva,

$$
y_{1}=\frac{-\sqrt{b^{2}-a^{2}}+\sqrt{b^{2}-a^{2}+4 c}}{2} .
$$

$\mathrm{Na}$ escolha de $\theta_{2}$, 


$$
\operatorname{sen}(T y)=\operatorname{sen} \theta_{2}=-\sqrt{1-\left(\frac{a}{b}\right)^{2}}<0
$$

Novamente ficamos com $y^{2}-c-y \sqrt{b^{2}-a^{2}}=0$, e como $c>0$, temos uma única solução positiva

$$
y_{2}=\frac{\sqrt{b^{2}-a^{2}}+\sqrt{b^{2}-a^{2}+4 c}}{2} .
$$

Obtemos duas seqüências,

$$
T_{n, 1}=\frac{\theta_{1}+2 n \pi}{y_{1}}, \quad T_{n, 2}=\frac{\theta_{2}+2 n \pi}{y_{2}} \quad(n=1,2, \cdots),
$$

correspondendo a $y_{1}$ e $y_{2}$, respectivamente. Além disso, $y_{2}>y_{1}$ e $\frac{\theta_{1}}{\theta_{2}}<\frac{1}{3}$, para quaisquer $a$ e $b$, com $a+b<0,|b|>|a|$ e $b<0$. Podemos escolher $c>0$ suficientemente grande tal que

$$
\frac{1}{3}<\frac{y_{1}}{y_{2}}<1
$$

Logo, $\theta_{1} y_{2}<\frac{\theta_{2}}{3} y_{2}<\frac{\theta_{2}}{3} 3 y_{1}$, o que implica

$$
T_{0,1}=\frac{\theta_{1}}{y_{1}}<\frac{\theta_{2}}{y_{2}}=T_{0,2}
$$

Por (2.32) temos que, para $y=y_{1}$,

$$
\begin{aligned}
\frac{d}{d T}(\operatorname{Re}(z)) & =-b y_{1}^{2}\left[a\left(\frac{-a}{b}\right)-\frac{2 y_{1}}{|b|} \sqrt{b^{2}-a^{2}}+b\right] D^{-1} \\
& =-y_{1}^{2}\left(-a^{2}+2 y_{1} \sqrt{b^{2}-a^{2}}+b^{2}\right) D^{-1} \\
& =y_{1}^{2}\left(-\left(b^{2}-a^{2}\right)-2 y_{1} \sqrt{b^{2}-a^{2}}\right) D^{-1}<0
\end{aligned}
$$

Concluímos que, todas as raízes que cruzam o eixo imaginário em $i y_{1}$, devem cruzar do lado esquerdo para o direito, conforme $T$ cresce, passando pelos instantes $T=$ $T_{n, 1}$.

Para $y=y_{2},(2.32)$ implica que 


$$
\begin{aligned}
\frac{d}{d T}(\operatorname{Re}(z)) & =-b{y_{2}}^{2}\left[a\left(\frac{-a}{b}\right)+\frac{2 y_{2}}{|b|} \sqrt{b^{2}-a^{2}}+b\right] D^{-1} \\
& =-y_{2}{ }^{2}\left(-a^{2}-2 y_{2} \sqrt{b^{2}-a^{2}}+b^{2}\right) D^{-1} \\
& =-y_{2}{ }^{2}\left(b^{2}-a^{2}-2 y_{2} \sqrt{b^{2}-a^{2}}\right) D^{-1}>0
\end{aligned}
$$

Portanto, as raízes que cruzam o eixo imaginário em $i y_{2}$, cruzam do lado esquerdo para o direito, conforme $T$ cresce, passando pelos valores $T=T_{n, 2}$.

Pelo Lema (2.2), a multiplicidade total das raízes, para as quais $\operatorname{Re}(z)>0$, é aumentada de dois quando $T$ cresce e passa por qualquer valor de $T_{n, 2}$, correspondendo à maior raiz $y_{2}$ e é diminuida de dois em qualquer valor $T_{n, 1}$, correspondendo à raiz $y_{1}$. Como a multiplicidade em $0 \leq T<T_{0,1}$ é dois (inicialmente temos instabilidade e devemos sempre considerar a solução e a sua conjugada), uma troca de estabilidade ocorre em $T_{0,1}$ e volta à instabilidade em $T_{0,2}$. Como os intervalos entre os valores subsequentes de $T$ entre os conjuntos é de $\frac{2 \pi}{y_{1}}>\frac{2 \pi}{y_{2}}$, somente um número finito de trocas de estabilidade pode ocorrer e para $T$ suficientemente grande a instabilidade prevalece.

(b) Seja $a<0$ e ainda $a+b<0$ e $|a|<|b| \Rightarrow b<0$.

Neste subcaso vale:

$$
\cos (T y)=-\frac{a}{b}<0
$$

e assim obtemos novamente: $\theta_{1}=\cos ^{-1}\left(-\frac{a}{b}\right)$, para $\frac{\pi}{2}<\theta_{1}<\pi$ e $\theta_{2}=\cos ^{-1}\left(-\frac{a}{b}\right)$, para $\pi<\theta_{1}<\frac{3 \pi}{2}$.

Para $\theta_{1}, \operatorname{sen}(T y)=\sqrt{1-\left(\frac{a}{b}\right)^{2}}>0$, substituindo na equação (2.29), ficamos $\operatorname{com} y^{2}-c+y \sqrt{b^{2}-a^{2}}=0$.

Como $c>0$, só existe uma raiz positiva, dada por

$$
y_{1}=\frac{-\sqrt{b^{2}-a^{2}}+\sqrt{b^{2}-a^{2}+4 c}}{2} .
$$

Na escolha de $\theta_{2}, \operatorname{sen}(T y)=\operatorname{sen} \theta_{2}=-\sqrt{1-(a / b)^{2}}<0$.

Novamente ficamos com $y^{2}-c-y \sqrt{b^{2}-a^{2}}=0$, e como $c>0$, temos uma única solução positiva 


$$
y_{2}=\frac{\sqrt{b^{2}-a^{2}}+\sqrt{b^{2}-a^{2}+4 c}}{2}
$$

$E$ assim, obtemos duas seqüências,

$$
T_{n, 1}=\frac{\theta_{1}+2 n \pi}{y_{1}}, \quad T_{n, 2}=\frac{\theta_{2}+2 n \pi}{y_{2}} \quad(n=1,2, \cdots),
$$

correspondendo a $y_{1}$ e $y_{2}$, respectivamente.

Para $y=i y,(2.32)$ implica que

$$
\begin{aligned}
\frac{d}{d T}(\operatorname{Re}(z)) & =-b y_{1}^{2}\left[a\left(\frac{-a}{b}\right) \frac{-2 y_{1}}{|b|} \sqrt{b^{2}-a^{2}}+b\right] D^{-1} \\
& =-y_{1}{ }^{2}\left(-a^{2}+2 y_{1} \sqrt{b^{2}-a^{2}}+b^{2}\right) D^{-1} \\
& =y_{1}{ }^{2}\left(-\left(b^{2}-a^{2}\right)-2 y_{1} \sqrt{b^{2}-a^{2}}\right) D^{-1}<0 .
\end{aligned}
$$

Logo, as raízes que cruzam o eixo imaginário em $i y_{1}$, cruzam do lado direito para o esquerdo, conforme $T$ cresce, passando pelos valores $T=T_{n, 1}$.

Para $y=y_{2}$, segue de (2.32) que

$$
\begin{aligned}
\frac{d}{d T}(R e(z)) & =-b{y_{2}}^{2}\left[a\left(\frac{-a}{b}\right)+2 \frac{y_{2}}{|b|} \sqrt{b^{2}-a^{2}}+b\right] D^{-1} \\
& =-y_{2}{ }^{2}\left(-a^{2}-2 y_{2} \sqrt{b^{2}-a^{2}}+b^{2}\right) D^{-1} \\
& =y_{2}{ }^{2}\left(-\left(b^{2}-a^{2}\right)+2 y_{2} \sqrt{b^{2}-a^{2}}\right) D^{-1}>0
\end{aligned}
$$

Logo, as raízes que cruzam o eixo imaginário em $i y_{2}$, cruzam do lado esquerdo para o direito, conforme $T$ cresce, passando pelos instantes $T=T_{n, 2}$.

A análise neste caso é semelhante ao subcaso (a), para quaisquer $a$ e $b$, com $a+b<0,|b|>|a|, b<0$ e $c>0$, podemos afirmar que, deve ocorrer um número finito de trocas de estabilidade e para $T$ suficientemente grande a instabilidade prevalece.

2) Consideremos $a+b>0$ e $c<0$.

Por (2.29), segue que $|a|<|b|$ e portanto $b>0$. Vejamos novamente duas situações:

(a) Seja $a>0$.

Então, $\cos (T y)=-\frac{a}{b}<0$, e assim temos $\theta_{1}=\cos ^{-1}\left(-\frac{a}{b}\right)$, com $\frac{\pi}{2}<\theta_{1}<\pi$ e 
$\theta_{2}=\cos ^{-1}\left(-\frac{a}{b}\right), \operatorname{com} \pi<\theta_{2}<\frac{3 \pi}{2}$.

$\mathrm{Na}$ escolha de $\theta_{1}$ :

$$
\operatorname{sen}(T y)=\sqrt{1-\left(\frac{a}{b}\right)^{2}}>0
$$

substituindo em (2.29), e como $b>0$, temos $y^{2}-y \sqrt{b^{2}-a^{2}}-c=0$, neste caso temos duas raízes positivas, já que $c<0$, dadas por

$$
\begin{aligned}
& y_{1}=\frac{\sqrt{b^{2}-a^{2}}+\sqrt{b^{2}-a^{2}+4 c}}{2} \\
& y_{2}=\frac{\sqrt{b^{2}-a^{2}}-\sqrt{b^{2}-a^{2}+4 c}}{2} .
\end{aligned}
$$

Na escolha de $\theta_{2}$ :

$$
\operatorname{sen}(T y)=\operatorname{sen} \theta_{2}=-\sqrt{1-\left(\frac{a}{b}\right)^{2}}<0
$$

Por (2.29) temos, $y^{2}-c+y \sqrt{b^{2}-a^{2}}=0$, e como $c<0$, não existem raízes positivas desta equação.

Obtemos duas seqüências,

$$
T_{n, 1}=\frac{\theta_{1}+2 n \pi}{y_{1}}, \quad T_{n, 2}=\frac{\theta_{1}+2 n \pi}{y_{2}}, \quad(n=1,2, \cdots)
$$

correspondendo às raízes positivas $y_{1}$ e $y_{2}$, respectivamente. Como $y_{1}>y_{2}$ e $\theta_{1} \leq \theta_{2}$ temos que $T_{0,1}<T_{0,2}$. Por $(2.32)$ e para $y=y_{1}$, temos que

$$
\begin{aligned}
\frac{d}{d T}(R e(z)) & =-b y_{1}{ }^{2}\left[a\left(\frac{-a}{b}\right)-2 \frac{y_{1}}{|b|} \sqrt{b^{2}-a^{2}}+b\right] D^{-1} \\
& =-y_{1}{ }^{2}\left(-a^{2}-2 y_{1} \sqrt{b^{2}-a^{2}}+b^{2}\right) D^{-1} \\
& =y_{1}{ }^{2}\left(-\left(b^{2}-a^{2}\right)+2 y_{1} \sqrt{b^{2}-a^{2}}\right) D^{-1}>0
\end{aligned}
$$


Logo, as raízes que cruzam o eixo imaginário em $i y_{1}$, devem cruzar do lado esquerdo para o direito, conforme $T$ cresce, passando pelos instantes $T=T_{n, 1}$.

Para $y=y_{2}$, vale $\cos (T y)=-a / b$ e $\operatorname{sen}(T y)=-\frac{\sqrt{b^{2}-a^{2}}}{|b|}$. Então, implica que

$$
\begin{aligned}
\frac{d}{d T}(\operatorname{Re}(z)) & =-b y_{2}{ }^{2}\left[a\left(\frac{-a}{b}\right)+2 \frac{y_{2}}{|b|} \sqrt{b^{2}-a^{2}}+b\right] D^{-1} \\
& =-y_{2}{ }^{2}\left(-a^{2}+2 y_{2} \sqrt{b^{2}-a^{2}}+b^{2}\right) D^{-1} \\
& =y_{2}{ }^{2}\left(-\left(b^{2}-a^{2}\right)-2 y_{2} \sqrt{b^{2}-a^{2}}\right) D^{-1}<0 .
\end{aligned}
$$

$\mathrm{E}$ assim, as raízes que cruzam o eixo imaginário em $i y_{2}$, devem cruzar do lado direito para o esquerdo, conforme $T$ cresce, passando pelos instantes $T=T_{n, 2}$.

Usando a prova da Proposição (2.3), concluímos que, pode ocorrer um número finito de trocas de estabilidade e para $T$ suficientemente grande a instabilidade prevalece.

(b) Seja $a<0$.

Nesta hipótese,

$$
\cos (T y)=-\frac{a}{b}>0
$$

e assim obtemos: $\theta_{1}=\cos ^{-1}\left(-\frac{a}{b}\right)$, para $0<\theta_{1}<\frac{\pi}{2}$ e $\theta_{2}=\cos ^{-1}\left(-\frac{a}{b}\right)$, para $\frac{3 \pi}{2}<\theta_{2}<2 \pi$.

Para $\theta_{1}$, temos sen $(T y)=\sqrt{1-\left(\frac{a}{b}\right)^{2}}>0$ e daí substituindo em (2.29), ficamos com $y^{2}-c-y \sqrt{b^{2}-a^{2}}=0$.

Como $c<0$ existem duas soluções positivas, dadas por

$$
\begin{aligned}
& y_{1}=\frac{\sqrt{b^{2}-a^{2}}+\sqrt{b^{2}-a^{2}+4 c}}{2}, \\
& y_{2}=\frac{\sqrt{b^{2}-a^{2}}-\sqrt{b^{2}-a^{2}+4 c}}{2} .
\end{aligned}
$$


$\mathrm{Na}$ escolha de $\theta_{2}$ :

$$
\operatorname{sen}(T y)=\operatorname{sen} \theta_{2}=-\sqrt{1-\left(\frac{a}{b}\right)^{2}}<0
$$

Então, de (2.29) segue que $y^{2}-c+y \sqrt{b^{2}-a^{2}}=0$, e como $c<0$, não existe raiz positiva.

Em seguida, obtemos duas seqüências:

$$
T_{n, 1}=\frac{\theta_{1}+2 n \pi}{y_{1}}, \quad T_{n, 2}=\frac{\theta_{1}+2 n \pi}{y_{2}} \quad(n=1,2, \cdots),
$$

correspondendo a $y_{1}$ e $y_{2}$, respectivamente.

Por (2.32) e para $y=y_{1}$, temos que

$$
\begin{aligned}
\frac{d}{d T}(R e(z)) & =-b y_{1}^{2}\left[a\left(\frac{-a}{b}\right)-2 \frac{y_{1}}{|b|} \sqrt{b^{2}-a^{2}}+b\right] D^{-1} \\
& =-y_{1}^{2}\left(-a^{2}-2 y_{1} \sqrt{b^{2}-a^{2}}+b^{2}\right) D^{-1} \\
& =y_{1}{ }^{2}\left(\left(b^{2}-a^{2}\right)+2 y_{1} \sqrt{b^{2}-a^{2}}\right) D^{-1}>0
\end{aligned}
$$

Logo, as raízes que cruzam o eixo imaginário em $i y_{1}$, devem cruzar do lado esquerdo para o direito, conforme $T$ cresce, passando pelos instantes $T=T_{n, 1}$.

Para $y=y_{2},(2.32)$ implica que

$$
\begin{aligned}
\frac{d}{d T}(R e(z)) & =-b y_{2}^{2}\left[a\left(\frac{-a}{b}\right)+\frac{2 y_{2}}{|b|} \sqrt{b^{2}-a^{2}}+b\right] D^{-1} \\
& =-y_{2}^{2}\left(-a^{2}+2 y_{2} \sqrt{b^{2}-a^{2}}+b^{2}\right) D^{-1} \\
& =y_{2}{ }^{2}\left(-\left(b^{2}-a^{2}\right)-2 y_{2} \sqrt{b^{2}-a^{2}}\right) D^{-1}<0
\end{aligned}
$$

Portanto, as raízes que cruzam o eixo imaginário em $i y_{2}$, devem cruzar do lado direito para o esquerdo, conforme $T$ cresce, passando pelos instantes $T=T_{n, 2}$.

Como $y_{1}>y_{2}$, segue que $\frac{2 \pi}{y_{1}}<\frac{2 \pi}{y_{2}}$ e $T_{0,1}<T_{0,2}$. Pela prova da Proposição (2.3), pode ocorrer um número finito de trocas de estabilidade e para $T$ suficientemente grande a instabilidade prevalece.

Conclusão: Desta análise, realizada para a equação (2.27), podemos concluir que: (1) ou a solução nula de (2.27) é estável para todo $T \geq 0$; 
(2) ou a solução nula é instável para todo $T \geq 0$;

(3) ou existem finitas trocas de estabilidade e para $T$ suficientemente grande a instabilidade prevalece.

A seguir, temos uma outra equação a qual será examinada da mesma forma que (2.27).

\section{Segunda Equação}

Consideremos a seguinte equação:

$$
\frac{d^{2} x}{d t^{2}}+a \frac{d x}{d t}+b x(t)+c x(t-T)=0
$$

onde $a, b$ e $c$ são constantes positivas e $T>0$ e portanto $b+c \neq 0$. Esta equação modela um processo com amortecimento instantâneo mas com força restauradora retardada.

A equação característica associada é dada por:

$$
z^{2}+a z+b+c e^{-T z}=0,
$$

onde $P(z)=z^{2}+a z+b$ e $Q(z)=c$ e assim o grau de $P$ é dois e o grau de $Q$ é zero.

Seja $z=i y$ uma raiz imaginária pura, $\operatorname{com} y>0$, e sendo $b+c \neq 0$, temos que $z=0$ não é uma raiz de (2.36). Substituindo iy em (2.36), obtemos:

$$
-y^{2}+a y i+b+\stackrel{\times}{x}(\cos (T y)-i \operatorname{sen}(T y))=0
$$

Tomando as partes real e imaginária, segue que

$$
\left\{\begin{array}{l}
y^{2}-b-c \cos (T y)=0, \\
a y-c \operatorname{sen}(T y)=0 .
\end{array}\right.
$$

consequentemente,

$$
\begin{cases}y^{2}-b & =c \cos (T y) \\ a y & =c \operatorname{sen}(T y)\end{cases}
$$

Elevando ao quadrado as duas igualdades acima e somando-as, obtemos 


$$
y^{4}+\left(a^{2}-2 b\right) y^{2}+b^{2}-c^{2}=0
$$

Nesta situação, $\Delta=\left(a^{2}-2 b\right)^{2}-4\left(b^{2}-c^{2}\right)$. Então, se $\Delta<0$, isto implica que não existe solução real e positiva da equação e portanto ela será instável para todo $T$, se for instável para $T=0$; ou ela será estável para todo $T$ se for estável para $T=0$. Se $\Delta \geq 0$, pode existir solução real positiva da equação acima.

Para $\Delta>0$, temos duas possibilidades:

1) se $2 b-a^{2}<0$ e $b^{2}>c^{2}$, neste caso, $\sqrt{\Delta}<\left|a^{2}-2 b\right|$ e portanto não existem raízes positivas;

2) se $2 b-a^{2}>0$ e $b^{2}>c^{2}$, neste caso $\sqrt{\Delta}<\left|a^{2}-2 b\right|=a^{2}-2 b$ e portanto existem duas raízes positivas.

Consideremos alguns casos, supondo $\Delta>0$.

\section{Primeiro Caso) $\quad b^{2}<c^{2}$.}

Neste caso, temos que $-4\left(b^{2}-c^{2}\right)>0$ e então $\sqrt{\Delta}>\left|a^{2}-2 b\right|$. Independente do sinal de $a^{2}-2 b$, sempre teremos $2 b-a^{2}+\sqrt{\Delta}>0$ e $2 b-a^{2}-\sqrt{\Delta}<0$ e assim, a única solução positiva possível de (2.38) é dada por

$$
y=\frac{1}{\sqrt{2}} \sqrt{2 b-a^{2}+\sqrt{\Delta}}
$$

Substituindo-a em (2.37), segue que

$$
c \cos (T y)=y^{2}-b=\frac{1}{2}\left(-a^{2}+\sqrt{\Delta}\right), \quad \operatorname{sen}(T y)=\frac{a y}{c}>0
$$

Essas equações determinam o sinal do $\cos (T y)$ e portanto o quadrante no qual $T y$ deve estar. Aplicando a função inversa do seno, obtemos a seqüência $\left\{T_{n}\right\}$

$$
T_{n}=\frac{1}{y}\left[\operatorname{sen}^{-1}\left(\frac{a y}{c}\right)+2 n \pi\right]
$$

para $n$ inteiro e $y$ solução positiva dada acima. Vale observar que a raiz $z=i y$ é simples.

Passemos agora a analisar a estabilidade. Primeiramente, vamos derivar ambos os membros da equação (2.36), em relação a $T$, 


$$
\begin{gathered}
2 z \frac{d z}{d T}+a \frac{d z}{d T}+c e^{-T y}\left(-z-T \frac{d z}{d T}\right)=0 \\
\frac{d z}{d T}\left(2 z+a-T c e^{-T z}\right)-z c e^{-T z}=0 \\
\frac{d z}{d T}=\frac{z c e^{-T z}}{2 z+a-T c e^{-T z}}=\frac{z c}{2 z e^{T z}+a e^{T z}-T c} .
\end{gathered}
$$

$\operatorname{Em} z=i y$

$$
\frac{d}{d T}(R e(z))=\operatorname{Re}\left(\frac{d z}{d T}\right)=\frac{c y(2 y \cos (T y)+a \operatorname{sen}(T y))}{(a \cos (T y)-2 y \operatorname{sen}(T y)-c T)^{2}+(2 y \cos (T y)+a \operatorname{sen}(T y))^{2}}
$$

Substituindo $\operatorname{sen}(T y)=\frac{a y}{c} \mathrm{e} \cos (T y)=\frac{y^{2}-b}{c}$, obtemos

$$
\frac{d}{d T}(\operatorname{Re}(z))=\frac{y^{2} c^{2}\left(2 y^{2}-2 b+a^{2}\right)}{\left(a b+a y^{2}+c^{2} T\right)^{2}+\left(2 y^{3}-2 b y+a^{2} y\right)^{2}}
$$

Desde que $2 y^{2}=2 b-a^{2}+\sqrt{\Delta}$, segue que $y^{2} c^{2}\left(2 b-a^{2}+\sqrt{\triangle}-2 b+a^{2}\right)=y^{2} c^{2} \sqrt{\triangle}>0$ e portanto,

$$
\frac{d}{d T}(\operatorname{Re}(z))>0
$$

Assim, conforme $T$ cresce, raízes cruzam o eixo imaginário e este cruzamento se dá do lado esquerdo para o direito, consequentemente, se solução nula for estável em $T=0$, a estabilidade é perdida em $T=T_{0}$ e a instabilidade persiste para todo $T$ grande.

Segundo Caso) $\quad b^{2}>c^{2}$, ainda no caso $\Delta>0$.

Neste caso vale $\sqrt{\Delta}<2 b-a^{2}$, portanto temos duas soluções positivas de (2.38) dadas por:

$$
y_{1}=\frac{1}{\sqrt{2}} \sqrt{2 b-a^{2}+\sqrt{\Delta}}
$$

e

$$
y_{2}=\frac{1}{\sqrt{2}} \sqrt{2 b-a^{2}-\sqrt{\Delta}}
$$


Como no caso anterior, temos que sen $\left(T y_{1}\right)=\frac{a y_{1}}{c}>0$ e $\operatorname{sen}\left(T y_{2}\right)=\frac{a y_{2}}{c}>0$. Assim, (2.37) implica que $c \cos \left(T y_{1}\right)=y_{1}{ }^{2}-b=\frac{1}{2}\left(-a^{2}+\sqrt{\Delta}\right)<0$, já que $\sqrt{\Delta}<2 b-a^{2}$, logo $\Delta<\left(a^{2}-2 b\right)^{2} \leq a^{4}$ e $c \cos \left(T y_{2}\right)=y_{2}{ }^{2}-b=\frac{1}{2}\left(-a^{2}-\sqrt{\Delta}\right)<0$. Portanto, podemos encontrar

$$
T_{1}=\frac{1}{y_{1}}\left[\operatorname{sen}^{-1}\left(\frac{a y_{1}}{c}\right)+2 \pi\right]
$$

e

$$
T_{2}=\frac{1}{y_{2}}\left[\operatorname{sen}^{-1}\left(\frac{a y_{2}}{c}\right)+2 \pi\right]
$$

Sejam $\theta_{1}$ e $\theta_{2}$ determinados por

$$
c \cos \left(\theta_{1}\right)=\frac{1}{2}\left[-a^{2}+\sqrt{\Delta}\right], \quad c \cos \left(\theta_{2}\right)=\frac{1}{2}\left[-a^{2}-\sqrt{\Delta}\right],
$$

onde $\frac{\pi}{2}<\theta_{1} \leq \pi$ e $\frac{\pi}{2}<\theta_{2} \leq \pi$. Então, $T y=\theta+2 n \pi$ e os valores de $T$ para os quais existem raízes imaginárias são

$$
T_{n, 1}=\frac{\theta_{1}+2 n \pi}{y_{1}}, \quad T_{n, 2}=\frac{\theta_{1}+2 n \pi}{y_{2}}, \quad(n=0,1,2 \cdots),
$$

correspondentes a cada raiz $y_{1}$ e $y_{2}$, respectivamente.

Por (2.39), e para $z_{1}=i y_{1}, \frac{d}{d T}\left(\operatorname{Re}\left(z_{1}\right)\right)>0$, segue que as raízes que cruzam em $i y_{1}$ cruzam do lado esquerdo para o direito, conforme $T$ cresce, passando pelos instantes $T=T_{n, 1}$. Para $z_{2}=i y_{2}$ temos $\frac{d}{d T}\left(\operatorname{Re}\left(z_{2}\right)\right)<0$ o que implica que as rázes que cruzam em $i y_{2}$ cruzam do lado direito para o esquerdo, conforme $T$ cresce, passando pelos instantes $T=T_{n, 2}$.

Como $y_{1}>y_{2}$ e $\theta_{1} \leq \theta_{2}$ temos $T_{0,1}<T_{0,2}$ e também $2 \pi / y_{1}<2 \pi / y_{2}$. Portanto existe um inteiro $k$ tal que os $k$ primeiros termos nas duas seqüências alternam-se, em seguida dois termos na primeira seqüência ocorrem consecutivamente. Em cada valor $T=T_{n, 1}$, um par de raízes cruzam o eixo imaginário em $i y_{1}$ do lado esquerdo para o direito, e em $T=T_{n, 2}$ um par de raízes cruzam em $i y_{2}$ do lado direito para o esquerdo. Como vimos na Primeira Equação, aqui o mesmo fenômeno se repete, ou seja, ocorre um número finito de trocas de estabilidade e para $T$ suficientemente grande a instabilidade prevalece. 
Observação 2.3. No caso em que $b^{2}=c^{2}$ e ainda $\Delta>0$, temos uma única raiz positiva dada por $y=\sqrt{2 b-a^{2}}$ e então $\operatorname{sen}(T y)>0$ e o sinal do cosseno vai depender do quadrante. Assim, obtemos uma seqüência correspondente $\left\{T_{n}\right\}$ e usando as equações acima podemos ver que

$$
\frac{d}{d T}(R e(z))>0 .
$$

Portanto as raízes cruzam o eixo imaginário do lado esquerdo para o direito e existe instabilidade para todo $T$ depois do primeiro cruzamento.

No caso $\Delta=0$, podemos ter duas situações:

(i) Se $2 b-a^{2}<0$, não existe solução da equação (2.38);

(ii) Se $2 b-a^{2}>0$, então $y^{4}+\left(a^{2}-2 b\right) y^{2}+b^{2}-c^{2}=0$ implica que $y=\sqrt{\frac{2 b-a^{2}}{2}}$ é a única solução da equação. Usando (2.39), obtemos

$$
\frac{d}{d T}(R e(z))=0 .
$$

Novamente, temos uma situação onde é preciso calcular as derivadas superiores para descobrir se alguma raiz cruza o eixo imaginário em iy, como já foi dito não faremos esses cálculos neste trabalho.

Note que, se a força restauradora retardada $c$ é maior ou igual que a força restauradora instantânea $b$, como no caso 1 , a estabilidade é perdida em $T_{0}$ e nunca é recuperada. Contudo, se a força restauradora retardada for menor que a força restauradora instantânea, finitas trocas de estabilidade ocorrem antes da instabilidade prevalecer. Assim, se a Equação (2.35) for instável em $T=0$, permanecerá instável para todo $T$. Isto segue do fato que o menor valor de $T$ para o qual existe uma raiz imaginária pura é sempre associado com a maior freqüência, implicando um crescimento inicial de 2 para 4 da multiplicidade total das raízes que possuem partes reais positivas.

Conclusão: Desta análise, realizada para a equação (2.35), podemos concluir que:

(1) ou a solução nula de (2.35) é estável para todo $T \geq 0$;

(2) ou a solução nula é instável para todo $T \geq 0$;

(3) ou existem finitas trocas de estabilidade e para $T$ suficientemente grande 
a instabilidade prevalece.

\section{Terceira Equação}

Agora analisemos o caso geral, ou seja a equação (2.26).

A equação característica associada é:

$$
z^{2}+a z+c+(b z+d) e^{-T z}=0
$$

onde $P(z)=z^{2}+a z+c$ e $Q(z)=b z+d$ e assim grau de $P$ é dois e o grau de $Q$ é um.

Seja $z=$ iy uma raiz imaginária pura de $(2.40)$, com $y>0$. É claro que $z=0$ não pode ser uma raiz. Substituindo iy em (2.40), obtemos

$$
-y^{2}+a y i+b y i(\cos (T y)-i \operatorname{sen}(T y))+c+d(\cos (T y)-i \operatorname{sen}(T y))=0
$$

Tomando as partes real e imaginária,

$$
\begin{cases}c-y^{2}+b y \operatorname{sen}(T y)+d \cos (T y) & =0 \\ a y+b y \cos (T y)-d \operatorname{sen}(T y) & =0\end{cases}
$$

Agora, elevando ao quadrado as duas igualdades acima e somando-as, obtemos:

$$
y^{4}+\left(a^{2}-b^{2}-2 c\right) y^{2}+c^{2}-d^{2}=0
$$

e então, $\Delta=\left(a^{2}-b^{2}-2 c\right)^{2}-4\left(c^{2}-d^{2}\right) . \Delta<0$, implica que não existem raízes positivas da equação e portanto nenhuma troca de estabilidade é possível de ocorrer e assim ou a equação é estável ou instável para todo $T$. No caso de $\Delta>0$, temos

$$
y^{2}=\frac{1}{2}\left[2 c+b^{2}-a^{2} \pm \sqrt{\triangle}\right]
$$


E aqui existem, novamente, alguns casos de interesse.

Primeiro Caso) $\quad c^{2}<d^{2}$.

Então $-4\left(c^{2}-d^{2}\right)>0$ e portanto $\Delta>0$ e nesta condição existe uma única raiz positiva,

$$
y=\frac{1}{\sqrt{2}} \sqrt{2 c+b^{2}-a^{2}+\sqrt{\Delta}}
$$

Segundo Caso) $\quad c^{2}>d^{2}$.

Então $-4\left(c^{2}-d^{2}\right)<0$ e portanto $\sqrt{\Delta}<\left|2 c+b^{2}-a^{2}\right|$, logo temos duas soluções positivas se (i) $b^{2}-a^{2}+2 c>0$ e (ii) $\left(b^{2}-a^{2}+2 c\right)^{2}>4\left(c^{2}-d^{2}\right)$.

Nosso interesse está em saber o sinal da derivada de $\operatorname{Re}(z)$ com respeito a $T$ nos pontos onde $z$ é uma raiz imaginária pura. Derivando ambos os membros de (2.40) em relação a $T$, obtemos:

$$
\begin{gathered}
2 z \frac{d z}{d T}+a \frac{d z}{d T}+b e^{-T y}\left[\frac{d z}{d T}-z\left(z+T \frac{d z}{d T}\right)\right]-d e^{-T z}\left(z+T \frac{d z}{d T}\right)=0 \\
\frac{d z}{d T}\left(2 z+a+b e^{-T z}-z T b e^{-T z}-d T e^{-T z}\right)=z^{2} b e^{-T z}+z d e^{-T z} \\
\frac{d z}{d T}=\frac{z(z b+d)}{(2 z+a) e^{T z}+b-T(b z+d)}
\end{gathered}
$$

É fácil ver que todas as raízes imaginárias são simples (a menos que $a=b=c=$ $d=0$ ). Sendo $\left(\frac{d z}{d T}\right)^{-1}=\frac{1}{\frac{d z}{d T}}$, segue que

$$
\left(\frac{d z}{d T}\right)^{-1}=\frac{(2 z+a) e^{T z}+b}{z(z b+d)}-\frac{T}{z} .
$$


(2.40) implica que

$$
e^{T z}=\frac{-(b z+d)}{z^{2}+a z+c}
$$

Sabendo que $\operatorname{sign}(\operatorname{Re}(z))=\operatorname{sign}(\operatorname{Re}(1 / z))$, vamos calcular

$$
\operatorname{sign}\left\{\left.\frac{d}{d T} \operatorname{Re}(z)\right|_{z=i y}\right\}=\operatorname{sign}\left\{\left.\operatorname{Re}\left(\frac{d z}{d T}\right)^{-1}\right|_{z=i y}\right\} .
$$

Substituindo $e^{T z}$ na equação da inversa da derivada, obtemos

$$
\left(\frac{d z}{d T}\right)^{-1}=\frac{-(2 z+a)}{\left(z^{2}+a z+c\right) z}+\frac{b}{z(z b+d)}-\frac{T}{z}
$$

Como $\operatorname{Re}(z+w)=\operatorname{Re}(z)+\operatorname{Re}(w)$ e $T / z$ é imaginário puro, quando $z=i y$, temos que

$$
\operatorname{sign}\left\{\frac{d}{d T} \operatorname{Re}(z)\right\}=\operatorname{sign}\left\{\operatorname{Re}\left(\frac{-(2 z+a)}{\left(z^{2}+a z+c\right) z}\right)+\operatorname{Re}\left(\frac{b}{z(z b+d)}\right)\right\} .
$$

Substituindo $z=i y$, obtemos

$$
\frac{-(2 z+a)}{\left(z^{2}+a z+c\right) z}=\frac{-2 c+2 y^{2}+2 a y i}{\left(c-y^{2}\right)^{2}+a^{2} y^{2}}+\frac{a^{2} y^{2}+a\left(c y-y^{3}\right) i}{y^{2}\left(c-y^{2}\right)^{2}+a^{2} y^{4}} .
$$

Logo,

$$
\left.\operatorname{Re}\left(\frac{-(2 z+a)}{\left(z^{2}+a z+c\right) z}\right)\right|_{z=i y}=\frac{2 y^{2}-2 c+a^{2}}{\left(c-y^{2}\right)^{2}+a^{2} y^{2}}
$$

e

$$
\left.\operatorname{Re}\left(\frac{b}{(b z+d) z}\right)\right|_{z=i y}=\frac{-b^{2}}{d^{2}+b^{2} y^{2}}
$$

Juntando essas duas últimas equações, obtemos 


$$
\left.\operatorname{sign}\left(\frac{d}{d T} \operatorname{Re}(z)\right)\right|_{z=i y}=\operatorname{sign}\left(\frac{2 y^{2}-2 c+a^{2}}{\left(c-y^{2}\right)^{2}+a^{2} y^{2}}-\frac{b^{2}}{d^{2}+b^{2} y^{2}}\right)=\operatorname{sign}\left(2 y^{2}-2 c+a^{2}-b^{2}\right) .
$$

Assim, $2 y^{2}=\left(-a^{2}+b^{2}+2 c\right) \pm \sqrt{\Delta}$ implica que

$$
\operatorname{sign}\left\{\left.\frac{d}{d T}(\operatorname{Re}(z))\right|_{z=i y}\right\}=\operatorname{sign}( \pm \sqrt{\Delta})
$$

No caso 1), onde $c^{2}<d^{2}$, somente uma raiz imaginária existe e como foi visto acima o $\operatorname{sign}\left\{\left.\frac{d}{d T}(\operatorname{Re}(z))\right|_{z=i y}\right\}>0$ e então os cruzamentos no eixo imaginário ocorrem do lado esquerdo para o direito quando $T$ cresce, e a estabilidade é perdida no momento do primeiro cruzamento e nunca mais é recuperada, ou seja, a instabilidade prevalece.

No caso 2), onde temos duas soluções positivas

$$
y_{1}=\frac{1}{\sqrt{2}} \sqrt{2 c+b^{2}-a^{2}+\sqrt{\Delta}}
$$

e

$$
y_{2}=\frac{1}{\sqrt{2}} \sqrt{2 c+b^{2}-a^{2}-\sqrt{\Delta}}
$$

$\operatorname{com} y_{1}>y_{2} \mathrm{e}$

$$
\begin{aligned}
& \operatorname{sign}\left(\left.\frac{d}{d T}(\operatorname{Re}(z))\right|_{z=i y_{1}}>0\right. \\
& \operatorname{sign}\left(\left.\frac{d}{d T}(\operatorname{Re}(z))\right|_{z=i y_{2}}<0\right.
\end{aligned}
$$

segue da equação (2.41) que

$$
\operatorname{sen}(T y)=\frac{a y+b y \cos (T y)}{d}, \quad \cos (T y)=\frac{d\left(y^{2}-c\right)-y^{2} a b}{b^{2} y^{2}+d}
$$

e podemos considerar

$$
T=\frac{1}{y} \cos ^{-1}\left(\frac{d\left(y^{2}-c\right)-y^{2} a b}{b^{2} y^{2}+d^{2}}\right)
$$


e assim obtemos

$$
T_{n, 1}=\frac{1}{y_{1}} \cos ^{-1}\left(\frac{d\left(y_{1}^{2}-c\right)-y_{1}^{2} a b}{b^{2} y_{1}^{2}+d^{2}}\right)+\frac{2 n \pi}{y_{1}}
$$

e

$$
T_{n, 2}=\frac{1}{y_{2}} \cos ^{-1}\left(\frac{d\left(y_{2}^{2}-c\right)-y_{2}^{2} a b}{b^{2} y_{2}^{2}+d^{2}}\right)+\frac{2 n \pi}{y_{2}}
$$

com $n=0,1,2 \cdots$. Portanto, raízes cruzam o eixo imaginário em $i y_{1}$ do lado esquerdo para o direito, nos instantes $T=T_{n, 1}$, e raízes cruzam o eixo imaginário em $i y_{2}$ do lado direito para o esquerdo nos instantes $T=T_{n, 2}$. Se a equação for estável para $T=0$, então necessariamente $T_{0,1}<T_{0,2}$ (desde que a multiplicidade das raízes com partes reais positivas não pode tornar-se negativa) e como

$$
T_{n+1,1}-T_{n, 1}=\frac{2 \pi}{y_{1}}<\frac{2 \pi}{y_{2}}=T_{n+1,2}-T_{n, 2},
$$

somente um número finito de trocas de estabilidade é possível ocorrer e para $T$ suficientemente grande, a instabilidade prevalece. Se a equação (2.26) for instável em $T=0$, então uma ou mais trocas de instabilidade para estabilidade e para instabilidade pode ocorrer.

Note que para o caso $\Delta=0$, temos que $y^{2}=\frac{2 c+b^{2}-a^{2}}{2}$, assim se $2 c+b^{2}-a^{2} \leq 0$, não existe solução positiva da equação (2.42). Se $2 c+b^{2}-a^{2}>0$, então existe uma única solução positiva $y$ dessa equação. E como podemos ver por (2.43)

$$
\left.\frac{d}{d T}(\operatorname{Re}(z))\right|_{z=i y}=0
$$

Novamente nada podemos concluir, e se torna necessário calcular as derivadas de ordem superiores.

Conclusão Desta análise, realizada para a equação (2.26), podemos concluir que:

(1) ou a solução nula de (2.26) é estável para todo $T \geq 0$;

(2) ou a solução nula é instável para todo $T \geq 0$;

(3) ou existem finitas trocas de estabilidade e para $T$ suficientemente grande a instabilidade prevalece.

Podemos resumir toda a análise realizada neste capítulo através do seguinte teorema: 
Teorema 2.3. Considere qualquer equação diferencial linear de primeira ou segunda ordem, homogênea do tipo retardada. Então, a equação característica associada é do tipo (2.2), onde $P$ e $Q$ são polinômios com coeficientes reais, sendo que o grau de $P$ é menor ou igual a 2 , e são verdadeiras as seguintes afirmações:

a) Se não existirem raízes imaginárias da equação característica, a estabilidade da solução nula nunca muda quando T cresce de zero a infinito;

b) Se existir uma única raiz imaginária pura, e se a solução nula for instável, ela nunca se torna estável. Se ela for estável para $T=0$, então ela se torna instável para valores pequenos de $T$, para os quais uma raiz imaginária existe, e permanece instável para $T$ suficientemente grande;

c) Se existirem duas raízes imaginárias puras, iy $y_{1}$ e $i y_{2},\left|y_{1}\right|>\left|y_{2}\right|$ existem finitas trocas de estabilidade que podem ocorrer conforme $T$ cresce, e eventualmente, se torna instável. Além disso, existem dois conjuntos de valores de $T, T_{n, 1}$ e $T_{n, 2}$ correspondendo $a i y_{1}$ e $i y_{2}$, respectivamente $(n=1,2, \cdots)$. Esses conjuntos são obtidos pela seguinte forma:

$$
T_{n, 1}=T_{0,1}+\frac{2 n \pi}{y_{1}}, \quad T_{n, 2}=T_{0,2}+\frac{2 n \pi}{y_{2}} \quad n=0,1,2 \cdots .
$$

Conforme $T$ cresce, a multiplicidade total das raízes com $\operatorname{Re}(z)>0$ é aumentada de dois, sempre que $T$ passa por qualquer valor $T_{n, 1}$ e é diminuida de dois quando $T$ passa por qualquer valor de $T_{n, 2}$. Quando a solução nula for estável para $T=0$, $k$ trocas de estabilidade para instabilidade e para estabilidade ocorrem quando os parâmetros são tais que

$$
T_{0,1}<T_{0,2}<T_{1,1}<\ldots<T_{k-1,1}<T_{k-1,2}<T_{k, 1}<T_{k+1,1}<T_{k, 2} \ldots
$$

ou $k$ trocas de instabilidade para estabilidade e para instabilidade quando

$$
T_{0,2}<T_{0,1}<T_{1,2}<\ldots<T_{k-1,2}<T_{k-1,1}<T_{k, 1}<T_{k, 2}<T_{k+1,1} \ldots
$$

se a solução nula for instável para $T=0$.

\section{Prova:}

O caso em que o grau de $P$ é um segue do Lema (2.1). Para o caso da equação ser de segunda ordem, isto é, grau de $P$ igual a dois segue da análise feita acima: Primeira Equação, Segunda Equação e Terceira Equação.

Lema 2.3. Sejam $P$ e $Q$ funções polinomiais com coeficientes reais tais que, $P$ e $Q$ não possuem zeros imaginários comuns, $P$ possue grau dois e o grau de $Q$ é 
menor que o grau de $P$. Suponhamos que $P(z)+Q(z) e^{-T z}=0$ tenha $r$ trocas de estabilidade. Consideremos $\tilde{P}(z)+\tilde{Q}(z) e^{-T z}=0$ onde $\tilde{P}(z)=(z+1) P(z)$ e $\tilde{Q}(z)=(z+1) Q(z)$. Então, a nova equação tem exatamente $r$ trocas de estabilidade.

\section{Prova:}

Os polinômios $\tilde{P}(z)=(z+1) P(z)$ e $\tilde{Q}(z)=(z+1) Q(z)$, possuem coeficientes reais, não possuem raízes imaginárias comuns, e o grau de $\tilde{P}$ é três (igual ao grau de $P$ mais 1 ), e o grau de $\tilde{Q}$ continua sendo maior que o grau de $\tilde{P}$. Para a nova equação temos,

$\tilde{F}(y)=|i y+1|^{2}|P(i y)|^{2}-|i y+1|^{2}|Q(i y)|^{2}=\left(y^{2}+1\right)\left(|P(i y)|^{2}-|Q(i y)|^{2}\right)=\left(y^{2}+1\right) F(y)$.

Portanto $\tilde{F}(y)$ e $F(y)$ têm exatamente as mesmas raízes positivas e também com a mesma multiplicidade. Segue do Corolário (2.1) que as direções de cruzamento são as mesmas em cada raiz. E mais, por (2.6), temos que $\operatorname{sen} \tilde{T} y=\operatorname{sen}(T y)$ e vale o mesmo para o cosseno. Portanto, os "tempos $\tilde{T}$ de cruzamentos"são também os mesmos. Consequentemente a nova equação só pode ter também $r$ trocas de estabilidade.

A seguir, vamos demonstrar o resultado central do nosso trabalho, o Teorema $(2.2)$.

\section{Prova do Teorema (2.2):}

Vamos dividir a demonstração deste importante resultado em dois casos:

Primeiro caso) Se $P(z)$ e $Q(z)$ são funções polinomiais.

Note que se o resultado for válido para $P$ polinômio de grau menor ou igual a dois, segue do Lema (2.3) que é válido para qualquer polinômio. Assim, pelo Teorema (2.3) a conclusão deste teorema é válida.

Segundo caso) Se $P(z)$ e $Q(z)$ não são polinômios.

Se $T$ e iy satisfazem (2.2), o que implica que ocorre (2.3) e portanto $F(y)=0$, isto prova o teorema, parte $a$ ), a equação é estável para todo $T \geq 0$ ou é instável para todo $T \geq 0$, e assim não há cruzamentos.

Pela hipótese (v), $F(y)$ tem um número finito de zeros reais. Vamos denotar os zeros positivos, que nós supomos serem todos simples, por

$$
y_{1}>y_{2}>\cdots>y_{p}>0 \text {. }
$$


Raízes de (2.2) cruzam o eixo imaginário em $i y_{k}$ nos valores $T_{k, m}$, com $m=$ $1,2, \cdots$, determinados na Proposição (2.1) (Observe que não podemos usar (2.21) para mostrar que cruzamentos em $y_{1}$ são à direita e em $y_{2}$ são à esquerda e assim sucessivamente). Mas, cruzamentos em raízes simples adjacentes devem ter direções opostas, pois $F^{\prime}\left(y_{j}\right)$ e $F^{\prime}\left(y_{j+1}\right)$ têm sinais opostos, já que $F$ é uma função diferenciável de $\mathbb{R} \rightarrow \mathbb{R}$ e se as raízes satisfazem (2.44), então é óbvio que em uma raiz a função é crescente e na sua consecutiva é decrescente.

Como já vimos, a diferença entre os valores de $T$, nos cruzamentos de uma dada solução $y_{k}$ é

$$
T_{k, m+1}-T_{k, m}=\frac{2 \pi}{y_{k}}
$$

por $(2.44)$, segue que

$$
\frac{2 \pi}{y_{1}}<\frac{2 \pi}{y_{2}}<\ldots<\frac{2 \pi}{y_{p}}
$$

Portanto, cruzamentos ocorrem em $y_{1}$ com mais freqüencia do que os demais, depois o seguinte mais frequente é $y_{2}$ e o menos frequente é o último, no caso, $y_{p}$, quando $T \rightarrow \infty$.

Vamos provar a parte $b$ ) do Teorema. Suponhamos que a equação $F(y)=0$ tenha pelo menos uma raiz positiva e que cada raiz positiva seja simples.

Em $T=0$, pela hipótese (iv) podem existir raízes no semi-plano direito (e um número finito). Quando $T \rightarrow \infty$, um número maior de raízes cruzam em $y_{1}$ do que em $y_{2}$, um número maior de raízes cruzam em $y_{2}$ do que em $y_{3}$ e assim por diante. Os cruzamentos, em $y_{1}$ devem ser a direita, pois, se por acaso fossem à esquerda, devido ao fato que existem mais cruzamentos em $y_{1}$ do que em $y_{2}$, cuja direção é oposta, implicaria que para $T$ suficientemente grande existiria um número negativo de raízes no semi-plano direito, o que é absurdo.

Neste caso concluímos que, se as raízes forem e se estiverem ordenadas segundo (2.44), então cruzamentos em $y_{1}, y_{3}, \ldots$, devem ser à direita e cruzamentos em $y_{2}$, $y_{4}, \ldots$, devem ser à esquerda. Em cada cruzamento o número de raízes no semi-plano direito muda por dois, desde que raízes ocorrem em pares conjugados pela hipótese (ii). Portanto existe um $T^{*}$ satisfazendo $b$ ).

\section{Equações com vários retardos}

Equações diferenciais com vários retardos, e sistemas de equações com um ou mais retardos, dão origem a equações trancendentais parecidas com (2.2) mas con- 
tendo mais que uma função exponencial. A análise das raízes de tais equações como função dos retardos é difícil, e os resultados são complicados. Contudo, para algumas equações, informações podem ser obtidas aplicando o resultado principal, Teorema (2.2), para um retardo. Considere a equação:

$$
\sum_{j=0}^{L} P_{j}(z) e^{-\tau_{j} z}+Q(z) e^{-T_{z}}=0
$$

onde $0=\tau_{0}<\tau_{1}<\ldots<\tau_{L}$ e $T \geq 0$. Se tomarmos

$$
P(z)=\sum_{j=0}^{L} P_{j}(z) e^{-\tau_{j} z},
$$

então, (2.45) se encontra na forma (2.2). Se supusermos condições sobre $P_{j}$ e $Q$ que garantam as hipóteses do Teorema (2.2), as conclusões desse teorema podem ser aplicadas a (2.45). Quando $Q$ e $P_{j}$ são polinômios, obtemos o seguinte resultado.

Teorema 2.4. Sejam $Q$ e $P_{j},(j=0,1, \cdots, L)$, polinômios que não dependem de $\tau_{j}$ ou de $T$, e que satisfaçam as seguintes condições:

(i) e (iii) do Teorema (2.2);

(ii') $Q$ e cada $P_{j}(j=0,1, \cdots, L)$ são polinômios com coeficientes reais.

(iv') O grau de $P_{0}(z)$ é maior do que o grau de $P_{j}(z)$, para $(j=1, \cdots, L)$ e de $Q(z)$.

Então, as conclusões do Teorema (2.2) e da Proposição (2.1) são válidas para a equação (2.45).

\section{Prova:}

Para provarmos este teorema, basta mostrarmos que as hipóteses do Teorema (2.2) estão satisfeitas. De fato,

(i) está satisfeita por hipótese;

(ii) $Q$ e $P_{j}, j=0,1, \cdots, L$, são polinômios com coeficientes reais, pela hipótese (ii') segue que vale (ii);

(iii) está satisfeita por hipótese;

(iv) devemos provar que existe no máximo um número finito de raízes de (2.45) no semi-plano direito quando $T=0$. Quando $T=0$, ficamos com: 


$$
P_{0}(z) e^{-\tau_{0} z}+P_{1}(z) e^{-\tau_{1} z}+\cdots+P_{L}(z) e^{-\tau_{L} z}+Q(z)=0
$$

como, por hipótese, grau de $P_{0}$ é maior que o máximo dos graus de $Q, P_{j}$, com $j=1,2, \cdots, L$, e como a equação (2.45) é retardada, segue que (iv) está satisfeita.

(v) $F(y)$ tem no máximo um número finito de zeros reais. De fato, por (iv') temos que

$$
\frac{P_{j}(i y)}{P_{0}(i y)} \stackrel{y \rightarrow \pm \infty}{\longrightarrow} 0
$$

$\operatorname{para} j=1, \cdots, L \mathrm{e}$

$$
\frac{Q(i y)}{P_{0}(i y)} \stackrel{y \rightarrow \pm \infty}{\longrightarrow} 0
$$

já que o grau de $P_{0}$ é maior que os demais, por hipótese. Agora, como $F(y)=$ $|P(i y)|^{2}-|Q(i y)|^{2}$, temos que

$$
\frac{F(y)}{\left|P_{0}(i y)\right|^{2}}=\frac{|P(i y)|^{2}}{\left|P_{0}(i y)\right|^{2}}-\frac{|Q(i y)|^{2}}{\left|P_{0}(i y)\right|^{2}}=\frac{\left|P_{0}(i y)\right|^{2}+\cdots+\left|P_{L}(i y)\right|^{2}}{\left|P_{0}(i y)\right|^{2}}-\frac{|Q(i y)|^{2}}{\left|P_{0}(i y)\right|^{2}} \stackrel{y \rightarrow \pm \infty}{\longrightarrow} 1
$$

logo,

$$
\frac{F(y)}{\left|P_{0}(i y)\right|^{2}} \stackrel{y \rightarrow \pm \infty}{\longrightarrow} 1
$$

Desde que $P_{0}(i y)$ não pode ter zeros para $y$ arbitrariamente grande, segue que os zeros de $F(y)$ estão em um intervalo compacto e portanto (v) está satisfeita. Concluímos assim, que todas as hipóteses do Teorema (2.2) estão satisfeitas e portanto este teorema é válido. 


\section{Capítulo 3}

\section{Estabilidade Absoluta}

Nos resultados provados no Capítulo anterior, uma das possibilidades obtidas para a localização das raízes de (2.2) como função do parâmetro $T, 0 \leq T<\infty$, era que todas as raízes estivessem em $\operatorname{Re}(z)<0$ para $0 \leq T<\infty$. Neste caso, o equilíbrio cuja linearização leva à equação característica (2.2) é assintoticamente estável para todos os retardos $T$. Dizemos, neste caso que o equilíbrio é absolutamente estável.

Ficamos motivados com o Artigo [2], já que este contém um importante resultado relacionado com o Teorema (2.2), cuja demonstração é feita com argumentos utilizados no Capítulo 2, juntamente com técnicas de Análise Complexa.

Definição 3.1. Um ponto equilibrio de uma equação com retardamento é dito ser absolutamente estável se é assintoticamente estável para todos os retardos, ou seja, a equação característica associada possue todas as suas raízes no semi-plano esquerdo, $\operatorname{Re}(z)<0$, para qualquer retardo $T, 0 \leq T<\infty$.

Teorema 3.1. Sejam $P(z)$ e $Q(z)$ funções analíticas em algum conjunto aberto contendo $\operatorname{Re}(z) \geq 0$ e que satisfaçam as seguintes condições:

(i) $P(z)$ e $Q(z)$ não possuem zeros comuns em $\operatorname{Re}(z) \geq 0$;

(ii) $P(0)+Q(0) \neq 0$;

(iii) $\overline{P(-i y)}=P(i y), \overline{Q(-i y)}=Q(i y)$;

(iv) Existe no máximo um número finito de valores reais de y tal que $|P(i y)|=|Q(i y)|$;

(v) $\lim _{\substack{|z| \rightarrow \infty \\ R e(z) \geq 0}}\left|\frac{Q(z)}{P(z)}\right|=0$.

Então, a equação (2.2) é absolutamente estável se, e somente se, $P(z)$ não possue zeros em $\operatorname{Re}(z) \geq 0$ e $|Q(i y)|<|P(i y)|$, para $0 \leq y<\infty$. 


\section{Prova:}

Suponhamos inicialmente que $P(z)$ não tenha zeros em $\operatorname{Re}(z) \geq 0$ e portanto a função $\frac{Q(z)}{P(z)}$ é analítica em $\operatorname{Re}(z) \geq 0$, pois $Q(z)$ e $P(z)$ são analíticas por hipótese. Logo, em um semi-círculo suficientemente grande $|z|=R$ em $R e(z) \geq 0$, temos pela hipótese (v) que

$$
\left|\frac{Q(z)}{P(z)}\right| \leq \rho<1
$$

Se também valer $|Q(i y)|<|P(i y)|$ para $0 \leq y<\infty$, então $\left|\frac{Q(z)}{P(z)}\right| \leq \rho<1$ no segmento de reta $z=i y,-R \leq y \leq R$. Assim, pelo Princípio do Máximo vale $\left|\frac{Q(z)}{P(z)}\right|<1$ em cada semi-círculo suficientemente grande no semi-plano direito. Mas $\left|e^{-T z}\right| \leq 1$ para $z$ no semi-plano direito e desde que (2.2) equivale a

$$
e^{T z}=-\frac{Q(z)}{P(z)}
$$

temos que, esta igualdade não ocorre para $z$ no semi-plano direito e portanto não pode existir raiz de (2.2) no semi-plano direito para qualquer $T \geq 0$.

Reciprocamente, se a condição $|Q(i y)|<\mid P(i y)$ não estiver satisfeita para algum $y$, então pelo Teorema (2.2) devem existir valores de $T$ para os quais (2.2) tem raízes em $R e(z) \geq 0$ e portanto (2.2) não poderá ser absolutamente estável. Assim, se $|Q(i y)|<|P(i y)|$ e então $|Q(z)|<|P(z)|$ em qualquer semi-círculo grande contido no semi-plano direito, $\left|Q(z) e^{-T z}\right| \leq|Q(z)|<|P(z)|$, logo, pelo Teorema de Rouché as funções $P(z)$ e $P(z)+Q(z) e^{-T z}$ possuem o mesmo número de zeros neste semicírculo. Portanto, se $P(z)$ tem zeros em $R e(z) \geq 0$, a equação (2.2) não poderá ser absolutamente estável, e isso completa a demonstração.

Vejamos um exemplo clássico, onde aplicamos o método acima.

Exemplo 3.1. Consideremos agora a seguinte equação característica:

$$
z^{2}+a z+b+c z e^{-T z}=0 \text {. }
$$

Notemos que essa equação é do tipo (2.28), estudada no Capítulo 2. Temos que, $P(z)=z^{2}+a z+b$ e $Q(z)=c z$. A condição $P(z) \neq 0$ para $z=x+i y$ em $\operatorname{Re}(z) \geq 0$, 
é calculada da seguinte maneira:

$$
(x+i y)^{2}+a(x+i y)+b=0,
$$

igualando partes real e imaginária,

$$
\left\{\begin{array}{l}
x^{2}-y^{2}+a x+b=0 \\
2 x y+a y=0
\end{array}\right.
$$

$A$ última equação implica que $y=0$ ou $x=-a / 2$. Se $y=0$, então, $x^{2}+a x+b=0$, o que implica $x=\frac{-a \pm \sqrt{a^{2}-4 b}}{2}$.

Assim, para que $x<0$, devemos impor que $a>0$ e também $b=y^{2}+a^{2} / 4>0$ independente de a. Concluímos que, para $P(z) \neq 0, z$ em $\operatorname{Re}(z) \geq 0$, devemos ter $a, b>0$.

Vejamos a condição $|Q(i y)|<|P(i y)|$, onde $P(i y)=-y^{2}+b+a y i$ e $Q(i y)=i y c$;

$$
|P(i y)|^{2}>|Q(i y)|^{2} \Rightarrow\left(b^{2}-y^{2}\right)^{2}+a^{2} y^{2}>y^{2} c^{2} \Rightarrow y^{4}+\left(a^{2}-c^{2}-2 b\right) y^{2}+b^{2}>0
$$

Sabemos que vale:

Observação 3.1. Um polinômio, do tipo $F(y)=y^{4}+\beta y^{2}+\gamma$, é positivo para $0 \leq y<\infty$ se, e somente se, $\gamma>0,(F(0)=\gamma>0)$ e $\Delta=\beta^{2}-4 \gamma<0$, então $F(y)=0$ não possue solução real ou $\gamma>0, \Delta=\beta^{2}-4 \gamma \geq 0$ e $\beta>0$.

Assim, pela Observação (3.1), temos duais possibilidades:

$$
\left(a^{2}-c^{2}-2 b\right)^{2}-4 b^{2}=\left(a^{2}-c^{2}\right)\left(a^{2}-c^{2}-4 b\right)<0,
$$

ou

$$
\left(a^{2}-c^{2}-2 b\right)>0 \quad e \quad\left(a^{2}-c^{2}\right)\left(a^{2}-c^{2}-4 b\right)>0 .
$$

Portanto, a solução da equação diferencial com retardamento associada a (3.1) será absolutamente estável se, e só se, $a>0, b>0$, e, ou $0<a^{2}-c^{2}<4 b$, ou $a^{2}-c^{2}>4 b$, pois, sendo $b>0$, vale $a^{2}-c^{2}-2 b<a^{2}-c^{2}$. 
Observação 3.2. Vale notar que, a condição $P(0)+Q(0) \neq 0$ no Teorema (3.1) implica que $z=0$ não é uma raiz de (2.2) para qualquer $T \geq 0$. Se $z=0$ for uma solução da equação característica então o equilibrio da equação diferencial com retardamento associada não é assintoticamente estável. Contudo, em alguns casos a equação característica contém um fator $z$, e daí uma integração a mais pode resolver o problema. Vejamos um exemplo simples deste fato.

Consideremos a equação

$$
z^{2}+c z+\left(1-e^{-T z}\right)(a z+b)=0
$$

que descreve o tempo de maturação num modelo de estrutura de população. Aqui, $P(z)=z^{2}+(a+c) z+b$ e $Q(z)=-a z-b$. Logo $P(0)+Q(0)=b-b=0 . E a$ forma da equação (3.2) é

$$
z+c+\left(\frac{1-e^{-T z}}{z}\right)(a z+b)=0
$$

Note que $\left(\frac{1-e^{-T z}}{z}\right)$ é analítica em $\operatorname{Re}(z) \geq 0$, mas essa equação não está na forma (2.2).

Para analisarmos a equação (3.3) e outras equações que podem ser colocadas na forma (2.2) por multiplicação, por um fator de $z$, é importante termos em mão um teste de estabilidade para tais casos. O próximo resultado fornece condições para analisarmos estas situações quanto a estabilidade absoluta.

Teorema 3.2. Sejam $P(z)$ e $Q(z)$ analíticas em algum conjunto aberto contendo $\operatorname{Re}(z) \geq 0$, satisfazendo as seguintes condições:

(a) $P(z)$ e $Q(z)$ não possuem zeros comuns em $\operatorname{Re}(z) \geq 0$;

(b) $\overline{P(-i y)}=P(i y), \overline{Q(-i y)}=Q(i y)$ para $y$ real;

(c) $P(0)+Q(0)=0$;

(d) $|P(i y)|>|Q(i y)|$ para $0<y<\infty$;

(e) $\lim _{\operatorname{Re}(z) \geq 0}\left|\frac{Q(z)}{P(z)}\right|=0$;

(f) $P(z) \neq 0$ para $\operatorname{Re}(z) \geq 0$.

Então, exceto para a raiz $z=0$, todas as raízes de (2.2) estão em $\operatorname{Re}(z)<0$, 
para $0 \leq T<\infty$

\section{Prova:}

Pela hipótese $(\mathrm{f})$ temos que $P(0) \neq 0$. A idéia da prova é aplicarmos o Teorema (3.1), para a equação

$$
P(z)+\epsilon P(0)+Q(z) e^{-T z}=0
$$

para $\epsilon$ suficientemente pequeno e positivo.

Para esta nova equação temos que, $P(0)+\epsilon P(0)+Q(0) e^{-T .0}=\epsilon P(0) \neq 0$ e mais $|Q(0)|=|P(0)|<(1+\epsilon)|P(0)|$. Assim, se chamarmos $P_{1}(z)=P(z)+\epsilon P(0)$ e $Q_{1}(z)=Q(z)$, estamos na forma (2.2). Mostremos que as hipóteses do Teorema (3.1) estão satisfeitas. De fato,

(i) Podemos escolher $\epsilon$ de modo que $P_{1}$ e $Q_{1}$ não tenham zeros comuns em $\operatorname{Re}(z) \geq 0$, já que $P$ e $Q$ não tem, por hipótese. Se $P_{1}\left(z_{0}\right)=Q_{1}\left(z_{0}\right)$, então $P\left(z_{0}\right)+\epsilon P(0)=Q\left(z_{0}\right)$, quando $\epsilon \rightarrow 0$, e temos que $P\left(z_{0}\right)=Q\left(z_{0}\right)$, o que é absurdo.

(ii) É claro, como já foi visto, $P_{1}(0)+Q_{1}(0) \neq 0$;

(iii) $\overline{P_{1}(-i y)}=\overline{P(-i y)+\epsilon P(0)}=P(i y)+\epsilon P(0)=P_{1}(i y)$. É imediato que $\overline{Q_{1}(-i y)}=Q_{1}(i y)$, para $y \in \mathbb{R}$;

(iv) $\left|P_{1}(i y)\right|=|P(i y)+\epsilon P(0)|=|Q(i y)|$, pela hipótese (d) temos que $|P(i y)|>|Q(i y)|$ para $0<y<\infty$ e $|P(i y)+\epsilon P(0)|-|Q(i y)| \geq|P(i y)|-|Q(i y)|>0$ quando $\epsilon \rightarrow 0$, portanto no limite esta condição está satisfeita.

(v)

$$
\lim _{\substack{|z| \rightarrow \infty \\ R e(z) \geq 0}}\left|\frac{Q_{1}(z)}{P_{1}(z)}\right|=\lim _{|z| \rightarrow \infty}\left|\frac{Q(z)}{P(z)+\epsilon P(0)}\right|
$$

para $\epsilon$ suficientemente pequeno segue da hipótese (e) que esse limite é nulo.

Assim, todas as hipóteses do Teorema (3.1) estão satisfeitas e portanto as raízes de (3.4) estão em $\operatorname{Re}(z)<0$ para $\epsilon$ suficientemente pequeno. Como as raízes de (3.4) dependem continuamente de $\epsilon$, as raízes de (2.2) devem estar em $\operatorname{Re}(z) \leq 0$. Contudo, a condição (d) garante que não existem raízes de (2.2) da forma $z=i y$ com $y>0$. Portanto, exceto para a raiz $z=0$, todas as raízes de(2.2) estão em $\operatorname{Re}(z)<0$.

Exemplo 3.2. Para a equação (3.2), a condição $P(z) \neq 0$ com $\operatorname{Re}(z) \geq 0$, se torna

$$
z^{2}+(a+c) z+b \neq 0 \text {. }
$$


Se $z=x+i y$ for uma raiz de $P$, vale:

$$
(x+i y)^{2}+(a+c)(x+i y)+b=0
$$

igualando partes real e imaginária, ficamos com:

$$
\begin{cases}x^{2}-y^{2}+(a+c) x+b & =0 \\ 2 x y+(a+c) y & =0\end{cases}
$$

Da última equação, segue que $y=0$ ou $x=-\frac{(a+c)}{2}$.

Se $y=0$, segue que $x^{2}+(a+c) x+b=0$, o que implica

$$
x=\frac{-(a+c) \pm \sqrt{(a+c)^{2}-4 b}}{2} .
$$

Logo, para $P(z) \neq 0$ com $\operatorname{Re}(z) \geq 0$, basta impormos que $(a+c)>0$ e $b>0$.

Pela expressão de $|P(i y)|^{2}$ e $|Q(i y)|^{2}$, segue que a condição $|Q(i y)|<|P(i y)|$, para $y>0$ equivale $a y^{4}+y^{2}\left(2 a c+c^{2}-2 b\right)>0$ e como $y^{2}>0$ isso se resume $a$ $y^{2}+2 a c+c^{2}-2 b>0$. Como essa desigualdade deve valer para todo $y>0$, segue que $b<a c+\frac{c^{2}}{2}$.

Portanto, todas as raízes de (3.2) estão em $\operatorname{Re}(z)<0$ para $0 \leq T<\infty$, exceto para a raiz simples $z=0$, se $a+c>0,0<b<a c+c^{2} / 2$. Isto dá uma condição de estabilidade absoluta para um certo tipo de equação cuja equação característica é do tipo (3.2). 


\section{Capítulo 4}

\section{Uma aplicação interessante}

O objetivo deste capítulo é aplicar a teoria desenvolvida no Capítulo 2 a um modelo que controla o mecanismo de respiração nos seres humanos e que consiste de um sistema de equações com retardamento. $O$ nosso objetivo é analisar este modelo quanto a estabilidade, possibilitando realizar significativa prevenção de irregularidades na respiração humana, assim como tratamentos desses problemas (entre eles, temos por exemplo, respiração periódica, apnea do sono, SIDS, etc). Uma abordagem detalhada deste modelo pode ser encontrado em [5].

Consideremos o sistema de equações diferenciais com retardamento não linear:

$$
\left\{\begin{array}{l}
\frac{d \tilde{x}}{d t}=p-\alpha W(\tilde{x}(t-\tau), \tilde{y}(t-\tau))\left(\tilde{x}(t)-x_{I}\right) \\
\frac{d \tilde{y}}{d t}=-\sigma+\beta W(\tilde{x}(t-\tau), \tilde{y}(t-\tau))\left(y_{I}-\tilde{y}(t)\right)
\end{array}\right.
$$

onde $\tilde{x}(\cdot)$ e $\tilde{y}(\cdot)$ denotam as concentrações arteriais de $\mathrm{CO}_{2}$ e $\mathrm{O}_{2}$, respectivamente; $W(\cdot, \cdot)$ é a função ventilação (o volume de gás movido pelo sistema respiratório); $\tau>0$ é o retardo do transporte; $x_{I}$ e $y_{I}$ são as concentrações inspiradas de $C O_{2} \mathrm{e}$ $\mathrm{O}_{2}$, respectivamente; $p$ é a taxa da produção de $\mathrm{CO}_{2} ; \sigma$ é a taxa consumida de $\mathrm{O}_{2} ; \alpha$ e $\beta$ são constantes positivas referentes a difusibilidade de $\mathrm{CO}_{2}$ e $\mathrm{O}_{2}$, respectivamente.

Agora, vamos colocar o sistema (4.1) em uma forma mais conveniente para a análise da estabilidade, introduzindo as novas variáveis:

$$
x(t)=a\left(\tilde{x}(t)-x_{I}\right), \quad y(t)=b\left(y_{I}-\tilde{y}(t)\right)
$$


onde $a$ e $b$ são constantes a serem determinadas. Substituindo em (4.1), obtemos:

$$
\frac{d x}{d t}=a \frac{d \tilde{x}}{d t}=a p-a \alpha W\left(x_{I}+\frac{x(t-\tau)}{a}, y_{I}-\frac{y(t-\tau)}{b}\right) \cdot\left(\frac{x(t)}{a}+x_{I}-x_{I}\right)
$$

ou seja,

$$
\frac{d x}{d t}=a p-a \alpha W\left(x_{I}+\frac{x(t-\tau)}{a}, y_{I}-\frac{y(t-\tau)}{b}\right) \frac{x(t)}{a} .
$$

O mesmo é feito para $y$, e obtemos:

$$
\frac{d y}{d t}=b \sigma-b \beta W\left(x_{I}+\frac{x(t-\tau)}{a}, y_{I}-\frac{y(t-\tau)}{b}\right) \frac{y(t)}{b} .
$$

Escolhendo as constantes $a$ e $b$ como $a=\frac{1}{p}$ e $b=\frac{1}{\sigma}$ ficamos com

$$
\begin{aligned}
& \frac{d x}{d t}=1-\alpha W\left(x_{I}+p x(t-\tau), y_{I}-\sigma y(t-\tau)\right) x(t), \\
& \frac{d y}{d t}=1-\beta W\left(x_{I}+p x(t-\tau), y_{I}-\sigma y(t-\tau)\right) y(t) .
\end{aligned}
$$

Se chamarmos $V(x, y)=W\left(x_{I}+p x, y_{I}-\sigma y\right)=W(u, v)$, então

$$
\left\{\begin{array}{l}
\frac{d x}{d t}=1-\alpha V(x(t-\tau), y(t-\tau)) x(t) \\
\frac{d y}{d t}=1-\beta V(x(t-\tau), y(t-\tau)) y(t)
\end{array}\right.
$$

Vamos trabalhar com este novo sistema, (4.2), buscar a solução equilíbrio e verificar quando esta será estável. Parece ser biologicamente realístico supor que 
$W(u, v)$ é crescente como função de $u$ e decrescente como função de $v$ para $u>x_{I}$ e $v<y_{I}$. Portanto, faremos as seguintes suposições:

(1) $V(x, y)$ será uma função diferenciável;

(2) $V(0,0)=W\left(x_{I}, y_{I}\right)=0$;

(3) $\frac{\partial V(x, y)}{\partial x}>0$ e $\frac{\partial V(x, y)}{\partial y}>0$, para $x, y>0$.

Note que a região $x>0$ e $y>0$ corresponde à região $\tilde{x}>x_{I}$ e $\tilde{y}<y_{I}$ nas variáveis originais. Para estudarmos a estabilidade do equilíbrio, vejamos alguns resultados necessários.

Teorema 4.1. Suponhamos que as hipóteses (1), (2) e (3) sejam válidas. Então, existe um único ponto de equilibrio positivo do sistema (4.2).

\section{Prova:}

A idéia da prova consiste em supor que esse ponto já exista e buscar uma expressão para ele. Assim, se existir um equilíbrio $\bar{x}$ e $\bar{y}$, segue imediatamente de (4.2) que $\bar{x} \neq 0$ e $\bar{y} \neq 0$. Mostremos que $\bar{x}=\frac{\beta \bar{y}}{\alpha}$. De fato, sendo $(\bar{x}, \bar{y})$ ponto equilíbrio vale

$$
\frac{d \bar{x}}{d t}=0=\frac{d \bar{y}}{d t} .
$$

Utilizando (4.2) temos

$$
\begin{aligned}
& 1-\alpha V(\bar{x}(t-\tau), \bar{y}(t-\tau)) \bar{x}(t)=0 \Rightarrow \bar{x}=\frac{1}{\alpha V(\bar{x}, \bar{y})} \\
& 1-\beta V(\bar{x}(t-\tau), \bar{y}(t-\tau)) \bar{y}(t)=0 \Rightarrow V(\bar{x}, \bar{y})=\frac{1}{\beta \bar{y}}
\end{aligned}
$$

Substituindo $V(\bar{x}, \bar{y})=V(\bar{x}(t-\tau), \bar{y}(t-\tau))$ na equação acima, ficamos com

$$
\bar{x}=\frac{1}{\alpha \frac{1}{\beta \bar{y}}} \Rightarrow \bar{x}=\frac{\beta \bar{y}}{\alpha},
$$


consequentemente

$$
\left\{\begin{array}{l}
\alpha V(\bar{x}, \bar{y})=\frac{1}{\bar{x}} \\
\beta V(\bar{x}, \bar{y})=\frac{1}{\bar{y}}
\end{array}\right.
$$

Como $V(0,0)=0$ e $V\left(\frac{\beta \bar{y}}{\alpha}, \bar{y}\right)$ é crescente em $\bar{y}$, existe uma única solução $\bar{y}$ de (4.3), devido ao Teorema do Valor Intermediário. Com isto definimos

$$
\bar{x}=\frac{\beta \bar{y}}{\alpha}
$$

e temos

$$
\alpha \bar{x} V(\bar{x}, \bar{y})=1=\beta \bar{y} V(\bar{x}, \bar{y})
$$

Vamos agora estudar a estabilidade assintótica do equilíbrio do sistema (4.2) sob as condições (1), (2) e (3). Chamando $\xi(t)=x(t)-\bar{x}$ e $\eta(t)=y(t)-\bar{y}$ em (4.3) e removendo os termos não lineares vamos obter um sistema linear.

De fato,

$$
\begin{aligned}
\frac{d \xi}{d t} & =\frac{d x}{d t}=1-\alpha V(\xi(t-\tau)+\bar{x}, \eta(t-\tau)+\bar{y})(\xi(t)+\bar{x}) \\
& =1+(-\alpha \xi(t)-\alpha \bar{x})[V((\bar{x}, \bar{y})+(\xi(t-\tau), \eta(t-\tau))] \\
& =1+(-\alpha \xi(t)-\alpha \bar{x})\left[V(\bar{x}, \bar{y})+V_{x} \xi(t-\tau)+V_{y} \eta(t-\tau)\right] \\
& =1-\alpha \xi(t) V(\bar{x}, \bar{y})-\alpha \xi(t) \bar{V}_{x} \xi(t-\tau)-\alpha \xi(t) \bar{V}_{y} \eta(t-\tau)-\alpha \bar{x} V(\bar{x}, \bar{y}) \\
& -\alpha \bar{x} \bar{V}_{x} \xi(t-\tau)-\alpha \bar{x} \bar{V}_{y} \eta(t-\tau) .
\end{aligned}
$$

Retirando a parte não linear e usando as propriedades do ponto equilíbrio, vale que

$$
\frac{d \xi}{d t}=-\alpha \xi(t) \bar{V}-\alpha \bar{x} \overline{V_{x}} \xi(t-\tau)-\alpha \bar{x} \overline{V_{y}} \eta(t-\tau)
$$

Usando os mesmos argumentos para $\frac{d \eta}{d t}$ obtemos

$$
\frac{d \eta}{d t}=-\beta \eta(t) \bar{V}-\beta \bar{y} \overline{V_{x}} \xi(t-\tau)-\beta \bar{y} \overline{V_{y}} \eta(t-\tau)
$$


Então podemos considerar:

$$
\left\{\begin{array}{l}
\frac{d \xi}{d t}=-\alpha \xi(t) \bar{V}-\alpha \bar{x} \overline{V_{x}} \xi(t-\tau)-\alpha \bar{x} \overline{V_{y}} \eta(t-\tau) \\
\frac{d \eta}{d t}=-\beta \eta(t) \bar{V}-\beta \bar{y} \overline{V_{x}} \xi(t-\tau)-\beta \bar{y} \overline{V_{y}} \eta(t-\tau)
\end{array}\right.
$$

onde $\bar{V}=V(\bar{x}, \bar{y}), \overline{V_{x}}=V_{x}(\bar{x}, \bar{y})$ e $\overline{V_{y}}=V_{y}(\bar{x}, \bar{y})$.

Assim, podemos escrever (4.4) da seguinte forma:

$$
\frac{d}{d t}\left(\begin{array}{l}
\xi(t) \\
\eta(t)
\end{array}\right)+A\left(\begin{array}{l}
\xi(t) \\
\eta(t)
\end{array}\right)+B\left(\begin{array}{l}
\xi(t-\tau) \\
\eta(t-\tau)
\end{array}\right)=\left(\begin{array}{l}
0 \\
0
\end{array}\right)
$$

onde $A=\left(\begin{array}{cc}\alpha \bar{V} & 0 \\ 0 & \beta \bar{V}\end{array}\right)$ e $B=\left(\begin{array}{cc}\alpha \bar{x} \overline{V_{x}} & \alpha \bar{x} \overline{V_{y}} \\ \beta \bar{y} \overline{V_{x}} & \beta \bar{y} \overline{V_{y}}\end{array}\right)$.

A equação característica associada é

$$
\Delta(\lambda, \tau)=\operatorname{det}\left(\lambda I+A+B e^{-\tau \lambda}\right)=P(\lambda)+Q(\lambda) e^{-\tau \lambda}=0 .
$$

De fato, somando as matrizes acima e calculando o determinante, obtemos

$$
\Delta(\lambda, \tau)=\lambda^{2}+\bar{V} \lambda(\alpha+\beta)+\alpha \beta \bar{V}^{2}+e^{-\tau \lambda}\left[\lambda\left(\beta \bar{y} \overline{V_{y}}+\alpha \bar{x} \overline{V_{x}}\right)+\alpha \beta \bar{V}\left(\bar{x} \overline{V_{x}}+\bar{y} \overline{V_{y}}\right)\right] .
$$

Logo,

$$
\begin{gathered}
P(\lambda)=\lambda^{2}+(\alpha+\beta) \bar{V} \lambda+\alpha \beta \bar{V}^{2}, \\
Q(\lambda)=\left(\alpha \bar{x} \overline{V_{x}}+\beta \bar{y} \overline{V_{y}}\right) \lambda+\alpha \beta \bar{V}\left(\bar{x} \overline{V_{x}}+\bar{y} \overline{V_{y}}\right) .
\end{gathered}
$$

Se fizermos $\tau=0,(4.5)$ se reduz a

$$
\Delta(\lambda, 0)=\lambda^{2}+\lambda\left(\alpha \bar{V}+\alpha \bar{x} \overline{V_{x}}+\beta \bar{V}+\beta \bar{y} \overline{V_{y}}\right)+\alpha \beta\left(\bar{V}^{2}+\bar{x} \bar{V} \overline{V_{x}}+\bar{y} \bar{V} \overline{V_{y}}\right)=0 .
$$

Pelas hipóteses assumidas (1), (2) e (3), temos $V_{x}>0, V_{y}>0, \bar{x}, \bar{y}>0, \alpha$ e $\beta$ são constantes positivas e portanto os coeficientes desta equação são positivos o que implica que as raízes têm parte real negativa.

Com o objetivo de estudar a estabilidade da equação linearizada vamos assumir as três hipóteses e vamos provar alguns fatos que resultarão na análise desejada. Primeiramente, vamos provar o seguinte lema. 
Lema 4.1. Se $\bar{V} \geq \bar{x} \overline{V_{x}}+\bar{y} \overline{V_{y}}$, então $\Delta(i w, \tau) \neq 0$ para $w \in \mathbb{R}, \tau \geq 0$. Se $\bar{V}<\bar{x} \overline{V_{x}}+\bar{y} \overline{V_{y}}$, então existe um único par de $w_{0}, \tau_{0}$, com $w_{0} \geq 0$ e $\tau_{0} \geq 0, w_{0} \tau_{0}<2 \pi$ tal que $\Delta\left(i w_{0}, \tau_{0}\right)=0$.

\section{Prova:}

Primeiramente, temos que

$$
\Delta(0, \tau)=\alpha \beta\left(\bar{V}^{2}+\bar{V} \bar{x} \overline{V_{x}}+\bar{y} \bar{V} \overline{V_{y}}\right) \neq 0
$$

pois $\alpha, \beta \neq 0, \bar{V} \neq 0,(\bar{x}, \bar{y}) \neq(0,0)$ e $V_{x}, V_{y}>0$

Além disso,

$$
\begin{aligned}
F(w) & =|P(i w)|^{2}-|Q(i w)|^{2} \\
& =\left|-w^{2}+\alpha \beta \bar{V}^{2}+(\alpha+\beta) \bar{V} w i\right|^{2}-\left|\left(\alpha \bar{x} \overline{V_{x}}+\beta \bar{y} \overline{V_{y}}\right) w i+\alpha \beta \bar{V}\left(\bar{x} \overline{V_{x}}+\bar{y} \overline{V_{y}}\right)\right|^{2} \\
& =w^{4}+w^{2}\left[\left(\alpha^{2}+\beta^{2}\right) \bar{V}^{2}-\left(\alpha \bar{x} \overline{V_{x}}+\beta \bar{y} \overline{V_{y}}\right)^{2}\right]+\alpha^{2} \beta^{2} \bar{V}^{2}\left[\bar{V}^{2}-\left(\bar{x} \overline{V_{x}}+\bar{y} \overline{V_{y}}\right)^{2}\right] .
\end{aligned}
$$

Portanto, $F(w)=w^{4}+k_{1} w^{2}+k_{2}$, onde

$$
\begin{gathered}
k_{1}=\left(\alpha^{2}+\beta^{2}\right) \bar{V}^{2}-\left(\alpha \bar{x} \overline{V_{x}}+\beta \bar{y} \overline{V_{y}}\right)^{2}, \\
k_{2}=\breve{V}^{2} \alpha^{2} \beta^{2}\left[\bar{V}^{2}-\left(\bar{x} \overline{V_{x}}+\bar{y} \overline{V_{y}}\right)^{2}\right] .
\end{gathered}
$$

Agora, se $\bar{V} \geq \bar{x} \overline{V_{x}}+\bar{y} \overline{V_{y}}$, então $\vec{V}^{2} \geq\left(\bar{x} \overline{V_{x}}+\bar{y} \overline{V_{y}}\right)^{2}$, segue que $k_{2} \geq 0$.

Também temos que,

$$
\begin{aligned}
\left(\alpha^{2}+\beta^{2}\right) \bar{V}^{2} & \geq\left(\alpha^{2}+\beta^{2}\right)\left(\bar{x} \overline{V_{x}}+\bar{y} \overline{V_{y}}\right)^{2} \\
& =\left(\alpha^{2}+\beta^{2}\right)\left(\bar{x}^{2} \bar{V}_{x}^{2}+2 \bar{x} \bar{y}{\overline{V_{x} V_{y}}}^{2} \bar{y}^{2}{\overline{V_{y}}}^{2}\right) \\
& =\alpha^{2} \bar{x}^{2}{\overline{V_{x}}}^{2}+\beta^{2} \bar{y}^{2}{\overline{V_{y}}}^{2}+\left[\left(\alpha^{2} \bar{y}^{2}{\overline{V_{x}}}^{2}+\beta^{2} \bar{x}^{2}{\overline{V_{x}}}^{2}\right)+2\left(\alpha^{2}+\beta^{2}\right) \overline{V_{x}} \bar{x} \overline{V_{y}} \bar{y}\right] \\
& >\alpha^{2} \bar{x}^{2}{\overline{V_{x}}}^{2}+\beta^{2} \bar{y}^{2}{\overline{V_{y}}}^{2}+2\left(\alpha^{2}+\beta^{2}\right) \overline{V_{x}} \bar{x} \overline{V_{y}} \bar{y} \\
& >\alpha^{2} \bar{x}^{2}{\overline{V_{x}}}^{2}+2 \alpha \beta \bar{x} \bar{y} \bar{V}_{y} \bar{V}_{x}+\beta^{2} \bar{y}^{2}{\overline{V_{y}}}^{2} \\
& =\left(\alpha \bar{x}{\overline{V_{x}}}^{2}+\beta \bar{y} \overline{V_{y}}\right)^{2}>0 .
\end{aligned}
$$

Assim, $k_{1}>0$ e $k_{2} \geq 0$, então $F(w)>0$, para $w \in \mathbb{R}, w \neq 0$, e por definição

$$
\Delta(i w, \tau)=P(i w)+Q(i w) e^{-i w \tau} \neq 0
$$


para $w \neq 0$, sempre que $\bar{V} \geq \bar{x} \overline{V_{x}}+\bar{y} \overline{V_{y}}$, então concluímos a primeira parte.

Provemos a segunda afirmação do Lema. Para isto suponhamos que

$$
\bar{V}<\bar{x} \overline{V_{x}}+\bar{y} \overline{V_{y}} .
$$

Consequentemente, $k_{2}<0$. Se $v=w^{2}$ em $F(w)=0$, segue que $v^{2}+k_{1} v+k_{2}=0$ o que implica

$$
v=\frac{-k_{1} \pm \sqrt{k_{1}^{2}-4 k_{2}}}{2}
$$

Como $k_{2}<0$, independente do sinal de $k_{1}$, temos que existe uma única raiz positiva, dada por

$$
v_{0}=\frac{-k_{1}+\sqrt{k_{1}^{2}-4 k_{2}}}{2}
$$

e daí $w_{0}=\sqrt{v_{0}}>0$. Portanto, existe uma única raiz $w_{0}$, tal que $\left|P\left(i w_{0}\right)\right|^{2}-$ $\left|Q\left(i w_{0}\right)\right|^{2}=0$, e, para este $w_{0}$, podemos determinar $\tau_{0}$, tal que $\tau_{0} w_{0}<2 \pi$. Isto completa a prova do lema.

Teorema 4.2. Se $\bar{V} \geq \bar{x} \overline{V_{x}}+\bar{y} \overline{V_{y}}$, então o equilibrio $(\bar{x}, \bar{y})$ é assintoticamente estável para todo retardo $\tau \geq 0$.

\section{Prova:}

Note que

$$
\Delta(\lambda, 0)=\lambda^{2}+\lambda\left(\alpha \bar{V}+\alpha \bar{x} \overline{V_{x}}+\beta \bar{V}+\beta \bar{y} \overline{V_{y}}\right)+\alpha \beta\left(\bar{x}^{2}+\bar{x} \bar{V} \overline{V_{x}}+\bar{y} \bar{V} \overline{V_{y}}\right) .
$$

Se chamarmos $A=\alpha \bar{V}+\alpha \bar{x} \overline{V_{x}}+\beta \bar{V}+\beta \bar{y} \overline{V_{y}}>0$ e $B=\alpha \beta\left(\bar{x}^{2}+\bar{x} \vec{V} \overline{V_{x}}+\bar{y} \bar{V} \overline{V_{y}}\right)>0$, então podemos escrever

$$
\lambda^{2}+A \lambda+B=0,
$$

para $\lambda=u+i v$, e igualando partes real e imaginária, ficamos com:

$$
\begin{cases}u^{2}-v^{2}+A u+B & =0 \\ 2 u v+v A & =0\end{cases}
$$

da última equação, segue que $v=0$ ou $u=\frac{-A}{2}<0$. Se $v=0$, então $u^{2}+A u+B=0$, implica

$$
u=\frac{-A \pm \sqrt{A^{2}-4 B}}{2}<0 \text {. }
$$


Logo, todas as raízes da equação tem partes reais negativas. Então, para $\tau=0$ o equilíbrio é estável. Agora, vamos aplicar o Teorema (2.2), parte a). Primeiro, vejamos se todas as condições exigidas estão satisfeitas. De fato,

(i) Segue de (4.10) que $P$ e $Q$ não possuem raízes imaginárias comuns;

(ii) $\overline{P(-i w)}=\overline{-w^{2}-(\alpha+\beta) \bar{V} i w+\alpha \beta \bar{V}^{2}}=P(i w)$. É fácil ver que $\overline{Q(-i w)}=Q(i w)$

(iii) $P(0)+Q(0)=\alpha \beta \bar{V}\left(\bar{V}+\left(\bar{x} \overline{V_{x}}+\bar{y} \bar{V} \overline{V_{y}}\right)\right) \neq 0$

(iv) Quando $\tau=0, P(\lambda)+Q(\lambda)=0$ só admite um número finito de raízes no semi-plano direito. Isto segue de (4.6) e (4.7).

(v) $F(w)=|P(i w)|^{2}-|Q(i w)|^{2}$ é um polinômio em $w$ e portanto possue um número finito de zeros reais.

Segue, portanto, do Teorema (2.2) que o equilíbrio é estável para todo $\tau \geq 0$.

Observação 4.1. Se $\bar{V}<\bar{x} \overline{V_{x}}+\bar{y} \overline{V_{y}}$, então segue do Lema (4.1) que

$$
\Delta(i w, \tau)=0
$$

com $w \in \mathbb{R}, \tau \geq 0$, se, e somente se, $w= \pm w_{0}, \tau=\tau_{n}=\tau_{0}+\frac{2 n \pi}{w_{0}}$, para $n=0,1,2 \ldots$, onde $w_{0}>0$ e $\tau_{0} \geq 0$, estão definidas no Lema (4.1).

Lema 4.2. $R e\left(i w_{0} \frac{\overline{\partial \Delta\left(i w_{0}, \tau_{n}\right)}}{\partial \lambda} Q\left(i w_{0}\right) e^{-i w_{0} \tau_{n}}\right)>0, \operatorname{para} n=0,1,2, \cdots$

\section{Prova:}

Sejam

$$
\begin{aligned}
& a_{1}=(\alpha+\beta) \bar{V} \\
& a_{2}=\alpha \beta \bar{V}^{2} \\
& b_{1}=\alpha \bar{x} \overline{V_{x}}+\beta \bar{y} \overline{V_{y}} \\
& b_{2}=\alpha \beta \bar{V}\left(\bar{x} \overline{V_{x}}+\bar{y} \overline{V_{y}}\right)
\end{aligned}
$$

e daí

$$
\Delta(\lambda, \tau)=\lambda^{2}+a_{1} \lambda+a_{2}+\left(b_{1} \lambda+b_{2}\right) e^{-\tau \lambda} .
$$


Assim,

$$
\Delta\left(i w_{0}, \tau_{n}\right)=-w_{0}^{2}+a_{1} w_{0} i+a_{2}+\left(b_{1} w_{0} i+b_{2}\right) e^{-\tau_{n} i w_{0}} .
$$

Derivando $\Delta$ em relação a $\lambda$, temos

$$
\frac{\partial \Delta(\lambda, \tau)}{\partial \lambda}=2 \lambda+a_{1}+b_{1} e^{-\tau \lambda}-\tau\left(\lambda b_{1}+b_{2}\right) e^{-\tau \lambda}
$$

Logo,

$$
\begin{aligned}
& \overline{\frac{\partial \Delta\left(i w_{0}, \tau_{n}\right)}{\partial \lambda}}=\overline{2 w_{0} i+a_{1}+b_{1} e^{-w_{0} i \tau_{n}}-\tau_{n}\left(i w_{0} b_{1}+b_{2}\right) e^{-\tau_{n} i w_{0}}} \\
& =-2 w_{0} i+a_{1}+b_{1} e^{w_{0} i \tau_{n}}-\tau_{n} \overline{Q\left(i w_{0}\right)} e^{\tau_{n} i w_{0}}
\end{aligned}
$$

Então,

$$
\begin{aligned}
i w_{0} & \frac{\overline{\partial \Delta\left(i w_{0}, \tau_{n}\right)}}{\partial \lambda} Q\left(i w_{0}\right) e^{-i w_{0} \tau_{n}}= \\
& =i w_{0}\left(-2 w_{0} i+a_{1}+b_{1} e^{w_{0} i \tau_{n}}-\tau_{n} \overline{Q\left(i w_{0}\right)} e^{\tau_{n} i w_{0}}\right) Q\left(i w_{0}\right) e^{-i w_{0} \tau_{n}} \\
& =i w_{0}\left[\left(-2 w_{0} i+a_{1}\right) Q\left(i w_{0}\right) e^{-i w_{0} \tau_{n}}+b_{1} Q\left(i w_{0}\right)-\tau_{n}\left|Q\left(i w_{0}\right)\right|^{2}\right] \\
& =i w_{0}\left(-2 w_{0} i+a_{1}\right) Q\left(i w_{0}\right) e^{-i w_{0} \tau_{n}}+b_{1} i w_{0} Q\left(i w_{0}\right)-i \tau_{n} w_{0}\left|Q\left(i w_{0}\right)\right|^{2} \\
& =i w_{0}\left(2 w_{0} i-a_{1}\right) P\left(i w_{0}\right)+i w_{0} b_{1} Q\left(i w_{0}\right)-\tau_{n} i w_{0}\left|Q\left(i w_{0}\right)\right|^{2} \\
& =i w_{0}\left(2 w_{0} i-a_{1}\right)\left(-w_{0}{ }^{2}+i w_{0} a_{1}+a_{2}\right)+i w_{0} b_{1} Q\left(i w_{0}\right)-i w_{0} \tau_{n}\left|Q\left(i w_{0}\right)\right|^{2}
\end{aligned}
$$

Portanto,

$$
\begin{aligned}
& i w_{0} \cdot \frac{\overline{\partial \Delta\left(i w_{0}, \tau_{n}\right)}}{\partial \lambda} Q\left(i w_{0}\right) e^{-i w_{0} \tau_{n}} \\
& =i w_{0}\left(2 w_{0} i-a_{1}\right)\left(-w_{0}{ }^{2}+i w_{0} a_{1}+a_{2}\right) \\
& \quad+i w_{0} b_{1}\left[i w_{0} b_{1}+b_{2}\right]-i w_{0} \tau_{n}\left|Q\left(i w_{0}\right)\right|^{2}
\end{aligned}
$$

Segue disso que

$$
R e\left(i w_{0} \cdot \frac{\overline{\partial \Delta\left(i w_{0}, \tau_{n}\right)}}{\partial \lambda} Q\left(i w_{0}\right) e^{-i w_{0} \tau_{n}}\right)=2 w_{0}^{2}\left(w_{0}{ }^{2}+\frac{1}{2}\left(a_{1}{ }^{2}-b_{1}{ }^{2}-2 a_{2}\right)\right)
$$


Pois,

$$
\begin{aligned}
i w_{0} \frac{\overline{\partial \Delta\left(i w_{0}, \tau_{n}\right)}}{\partial \lambda} Q\left(i w_{0}\right) e^{-i w_{0} \tau_{n}=} & i w_{0}\left(2 w_{0}-a_{1}\right)\left(-w_{0}^{2}+i w_{0} a_{1}+a_{2}\right)-i w_{0} \tau_{n}\left|Q\left(i w_{0}\right)\right|^{2} \\
& \left.+i w_{0} b_{1}\left(b_{1} i w_{0}+b_{2}\right)\right] \\
= & 2 w_{0}^{4}-2 w_{0}^{3} a_{1} i-2 w_{0}^{2} a_{2}+w_{0}^{3} a_{1} i+w_{0}^{2} a_{1}^{2}-w_{0} a_{1} a_{2} i \\
& -i \tau_{n} w_{0}\left|Q\left(i w_{0}\right)\right|^{2}-w_{0}^{2} b_{1}^{2}+w_{0} b_{1} b_{2} i
\end{aligned}
$$

portanto,

$$
R e\left[i w_{0} \cdot \frac{\overline{\partial \Delta\left(i w_{0}, \tau_{n}\right)}}{\partial \lambda} Q\left(i w_{0}\right) e^{-i w_{0} \tau_{n}}\right]=2 w_{0}^{4}-2 w_{0}^{2} a_{2}+a_{1}^{2} w_{0}^{2}-w_{0}^{2} b_{1}^{2}
$$

donde segue (4.14). Podemos verificar que

$$
\begin{aligned}
a_{1}^{2}-b_{1}^{2}-2 a_{2} & =(\alpha+\beta)^{2} \bar{V}^{2}-\left(\alpha \bar{x} \overline{V_{x}}+\beta \bar{y} \overline{V_{y}}\right)^{2}-2\left(\alpha \beta \bar{V}^{2}\right) \\
& =\left(\alpha^{2}+2 \alpha \beta+\beta^{2}\right) \bar{V}^{2}-2 \alpha \beta \bar{V}^{2}+\left(\alpha \bar{x} \overline{V_{x}}+\beta \bar{y} \overline{V_{y}}\right)^{2} \\
& =\left(\alpha^{2}+\beta^{2}\right) \bar{V}^{2}+\left(\alpha \bar{x} \overline{V_{x}}+\beta \bar{y} \overline{V_{y}}\right)^{2} \\
& =k_{1} .
\end{aligned}
$$

De (4.14) e (4.11), vem que

$$
\begin{aligned}
\operatorname{Re}\left(i w_{0} \cdot \frac{\overline{\partial \Delta\left(i w_{0}, \tau_{n}\right)}}{\partial \lambda} Q\left(i w_{0}\right) e^{-i w_{0} \tau_{n}}\right) & =2 v_{0}\left(v_{0}+\frac{1}{2} k_{1}\right) \\
& =2 v_{0}\left(-\frac{k_{1}}{2}+\frac{\sqrt{k_{1}^{2}-4 k_{2}}}{2}+\frac{k_{1}}{2}\right) \\
& =v_{0} \sqrt{k_{1}^{2}-4 k_{2}}>0
\end{aligned}
$$

Corolário 4.1. Suponhamos $\bar{V}<\bar{x} \overline{V_{x}}+\bar{y} \overline{V_{y}}$. Sejam $w_{0}, \tau_{n}, n=0,1,2, \cdots$, definidos como no Lema (4.1) e na Observação (4.1). Então, para cada $\tau_{n}$, existe uma vizinhança $I_{n} \subset \mathbb{R}$ de $\tau_{n}$ e uma função continuamente diferenciável $\lambda_{n}: I_{n} \longrightarrow C$, tal que:

(i) $\lambda_{n}\left(\tau_{n}\right)=i w_{0}$;

(ii) $\Delta\left(\lambda_{n}(\tau), \tau\right)=0$, com $\tau \in I_{n}$;

(iii) $\operatorname{Re}\left(\left.\frac{d \lambda_{n}(\tau)}{d \tau}\right|_{\tau=r_{n}}\right)>0$. 


\section{Prova:}

Segue da demonstração do Lema (4.2), que

$$
\frac{\partial \Delta\left(i w_{0}, \tau_{n}\right)}{\partial \lambda} \neq 0
$$

logo, podemos aplicar o Teorema da Função Implícita, o que garante que existe um aberto $I_{n}$ contendo $\tau_{n}$ e uma função continuamente diferenciável $\lambda_{n}$ tal que $\lambda_{n}\left(\tau_{n}\right)=i w_{0}$ e $\Delta\left(\lambda_{n}(\tau, \tau)\right)=0$ para $\tau \in I_{n}$ e portanto as conclusões (i) e (ii) já estão provadas.

Provemos agora (iii). Usando que $\Delta\left(\lambda_{n}(\tau)_{n}, \tau_{n}\right)=0$, temos que $\Delta\left(i w_{0}, \tau_{n}\right)=0$. Vamos derivar esta expressão em relação a $\tau$. Pelo Teorema da Função Implícita, segue que

$$
\frac{d \lambda_{n}}{d \tau}=\frac{-\frac{\partial \Delta}{\partial \tau}}{\frac{\partial \Delta}{\partial \lambda}} \Rightarrow \frac{d \lambda}{d \tau} \frac{\partial \Delta}{\partial \lambda}+\frac{\partial \Delta}{\partial \tau}=0
$$

como $\Delta(\lambda, \tau)=P(\lambda)+Q(\lambda) e^{-\tau \lambda}$, temos que

$$
\frac{\partial \Delta}{\partial \tau}=Q(\lambda) e^{-\tau \lambda}(-\lambda)
$$

no ponto $i w_{0}$, vale

$$
\frac{\partial \Delta}{\partial \tau}=Q\left(i w_{0}\right) e^{-i w_{0} \tau_{n}}\left(-i w_{0}\right)
$$

logo,

$$
\frac{\partial \Delta}{\partial \lambda}\left(i w_{0}, \tau_{n}\right) \frac{d \lambda_{n}(\tau)}{d \tau}-i w_{0} Q\left(i w_{0}\right) e^{-i w_{0} \tau_{n}}=0
$$


Utilizando agora o Lema (4.2) e a equação (4.17) temos que

$$
\begin{aligned}
& \frac{d \lambda_{n}}{d \tau}(\tau)=\frac{i w_{0} Q\left(i w_{0}\right) e^{-w_{0} i \tau_{n}}}{\frac{\partial \Delta\left(i w_{0}, \tau_{n}\right)}{\partial \lambda}} \cdot \frac{\frac{\overline{\partial \Delta\left(i w_{0}, \tau_{n}\right)}}{\partial \lambda}}{\frac{\partial \Delta\left(i w_{0}, \tau_{n}\right)}{\partial \lambda}}= \\
& \frac{i w_{0} \frac{\overline{\partial \Delta\left(i w_{0}, \tau_{n}\right)}}{\partial \lambda} Q\left(i w_{0}\right) e^{-i w_{0} \tau_{n}}}{\left|\frac{\partial \Delta\left(i w_{0}, \tau_{n}\right)}{\partial \lambda}\right|^{2}} .
\end{aligned}
$$

Portanto,

$$
\begin{aligned}
\operatorname{Re}\left(\frac{d \lambda_{n}(\tau)}{d \tau}\right)= & \left.\frac{\overline{\frac{\partial \Delta\left(i w_{0}, \tau_{n}\right)}{\partial \lambda}} Q\left(i w_{0}\right) e^{-i w_{0} \tau_{n}}}{\left|\frac{\partial \Delta\left(i w_{0}, \tau_{n}\right)}{\partial \lambda}\right|^{2}}\right)= \\
& \operatorname{Re}\left(\frac{i w_{0} \frac{1}{1}}{\left|\frac{\partial \Delta\left(i w_{0}, \tau_{n}\right)}{\partial \lambda}\right|^{2}} \operatorname{Re}\left(i w_{0} \frac{\frac{\partial \Delta\left(i w_{0}, \tau_{n}\right)}{\partial \lambda}}{\partial \lambda^{2}} Q\left(i w_{0}\right) e^{-i w_{0} \tau_{n}}\right)>0\right.
\end{aligned}
$$

Teorema 4.3. Suponhamos $\bar{V}<\bar{x} \overline{V_{x}}+\bar{y} \overline{V_{y}}$. Seja $\tau_{0}$ definido como na Observação (4.1). Então o equilíbrio $(\bar{x}, \bar{y})$ é assintoticamente estável se $0 \leq \tau<\tau_{0}$ e instável se $\tau>\tau_{0}=\tau^{*}$.

\section{Prova:}

Se $\bar{V}<\bar{x} \overline{V_{x}}+\bar{y} \overline{V_{y}}$ então existe uma raiz $w_{0}$ positiva e simples, como foi visto no Lema (4.1) de $F(w)=0$. Associada a esta raiz, existe uma seqüência $\tau_{n}$, tal que

$$
\operatorname{Re}\left(\left.\frac{d \lambda_{n}(\tau)}{d \tau}\right|_{\tau=\tau_{n}}\right)>0
$$


e assim pelo Teorema (2.2), parte b), segue este resultado.

Disso tudo concluímos que se o transporte do retardo do sistema é maior que $\tau_{0}$, então podemos esperar manifestação de irregularidades do sistema, já que teremos instabilidade. 


\section{Capítulo 5}

\section{Aplicações}

Neste capítulo, vamos aplicar os resultados obtidos nos Capítulos 2 e 3 ao estudo da estabilidade de alguns modelos físicos e biológicos.

Exemplo 5.1. Neste exemplo, vamos estudar o comportamento qualitativo, quando o retardo é tomado como um parâmetro, de uma classe de modelos matemáticos que surgem no estudo para o controle de síntese de proteínas.

Modelos matemáticos deste tipo, foram inicialmente propostos por Goodwin, [9] e [10].

A generalização $n$-dimensional, que estudamos aqui, para o modelo de Goodwin, é dada pelo seguinte sistema:

$$
\left\{\begin{array}{l}
\dot{x_{1}}(t)=\frac{a}{1+k\left[x_{n}\left(t-\tau_{1}\right)+\overline{x_{n}}\right]^{\rho}}-b\left[x_{1}(t)+\overline{x_{1}}\right] \\
\dot{x}_{i}(t)=\alpha_{i} x_{i-1}\left(t-\tau_{i}\right)-\beta_{i} x_{i}(t), \quad i=2, \cdots, n,
\end{array}\right.
$$

com constantes positivas $\rho, a, b, k, \alpha_{i}, \beta_{i}, \tau_{i}$, onde $\bar{x}=\left(\overline{x_{1}}, \ldots, \overline{x_{n}}\right)^{T}$ é a única solução equilíbrio de

$$
\left\{\begin{array}{l}
\dot{z}_{1}(t)=\frac{a}{1+k\left[z_{n}\left(t-\tau_{1}\right)\right]^{p}}-b z_{1}(t) \\
\dot{z}_{i}(t)=\alpha_{i} z_{i-1}\left(t-\tau_{i}\right)-\beta_{i} z_{i}(t), \quad i=2, \cdots, n .
\end{array}\right.
$$

Observemos que $\overline{x_{i}}>0$. Vamos utilizar em (5.1) a seguinte substituição, 


$$
\left\{\begin{array}{l}
\hat{x}_{1}(t)=x_{1}(t) \\
\hat{x}_{i}(t)=x_{i}\left(t+\sum_{j=2}^{i} \tau_{j}\right), \quad i=2, \cdots, n
\end{array}\right.
$$

Assim, podemos notar que

$$
\hat{x}_{n}(t)=x_{n}\left(t+\tau_{2}+\ldots+\tau_{n}\right) \Rightarrow \hat{x}_{n}\left(t-\tau_{1}-\tau_{2}-\ldots-\tau_{n}\right)=x_{n}\left(t-\tau_{1}\right)
$$

Se denotarmos $r=\sum_{j=1}^{n} \tau_{j}$, então podemos escrever

$$
\begin{gathered}
\dot{\hat{x}}_{1}(t)=\frac{a}{1+k\left[\hat{x}_{n}(t-r)+\overline{x_{n}}\right] \rho}-b\left[\hat{x}_{1}(t)+\overrightarrow{x_{1}}\right] \\
\dot{\hat{x}}_{2}(t)=\dot{x_{2}}\left(t+\tau_{2}\right)=\alpha_{2} x_{1}\left(t+\tau_{2}-\tau_{2}\right)-\beta_{2} x_{2}\left(t+\tau_{2}\right)=\alpha_{2} \hat{x}_{1}(t)-\beta_{2} \hat{x}_{2}(t) .
\end{gathered}
$$

Repetindo para os demais $x_{i}$, e voltando à notação inicial, ficamos com o seguinte sistema com um único retardo:

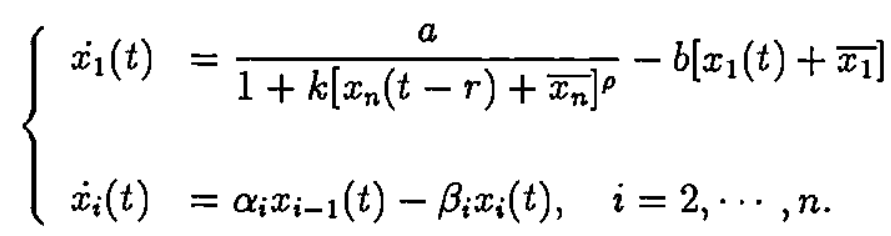

Vamos agora linearizar o sistema (5.2) em torno da solução $x(t) \equiv 0$, e colocá-lo na forma

$$
\dot{y}(t)=A y(t)+B y(t-r)
$$

Para isto, consideremos a seguinte função,

$$
f(\xi)=-b \overline{x_{1}}+\frac{a}{1+k\left[\xi+\overline{x_{n}}\right]} .
$$

Se denotarmos

$$
y(t)=\left(\begin{array}{c}
x_{1}(t) \\
\ldots \ldots \\
x_{n}(t)
\end{array}\right)
$$


e

$$
A=\left(\begin{array}{ccccc}
-b & 0 & \ldots & \ldots & 0 \\
\alpha_{2} & -\beta_{2} & 0 \ldots & 0 & \\
0 & \alpha_{3} & -\beta_{3} & \ldots & 0 \\
\ldots & \ldots & \ldots & \ldots & \ldots \\
0 & 0 & \ldots & \alpha_{n} & -\beta_{n}
\end{array}\right) \quad B=\left(\begin{array}{cccc}
0 & \ldots & 0 & f^{\prime}(0) \\
0 & \ldots & \ldots & 0 \\
\ldots \ldots & \ldots & \ldots \\
0 & 0 & \ldots & 0
\end{array}\right)
$$

obtemos (5.3). Para encontrarmos a equação característica, basta considerarmos

$$
\operatorname{det}\left[A-\lambda I+B e^{-\lambda r}\right]=0
$$

e resolvendo este determinante temos

$$
(-b-\lambda)\left[\prod_{j=2}^{n}\left(-\beta_{j}-\lambda\right)\right]+\left(\prod_{j=2}^{n} \alpha_{j}\right) f^{\prime}(0) e^{-\lambda r}(-1)^{n+1}=0
$$

portanto a equação característica é dada por

$$
(b+\lambda)\left[\prod_{j=2}^{n}\left(\beta_{j}+\lambda\right)\right]-\left(\prod_{j=2}^{n} \alpha_{j}\right) f^{\prime}(0) e^{-\lambda r}=0
$$

onde $\beta_{j}>0, \alpha_{j}>0, b>0$ e

$$
f^{\prime}(0)=\frac{-a \rho k{\overline{x_{n}}}^{\rho-1}}{\left[1+k{\overline{x_{n}}}^{\rho}\right]^{2}}<0
$$

Vejamos se as hipóteses do Teorema (2.2) estão satisfeitas. Neste caso,

$$
P(\lambda)=(b+\lambda)\left[\prod_{j=2}^{n}\left(\beta_{j}+\lambda\right)\right], \quad Q(\lambda)=-\left(\prod_{j=2}^{n} \alpha_{j}\right) f^{\prime}(0)>0
$$

Então:

(i) $P$ e $Q$ não possuem zeros imaginários comuns, pois que $Q$ é constante.

(ii) É fácil ver que $\overline{P(-i y)}=P(i y)$ e o mesmo para $Q$.

(iii) $P(0)+Q(0)=b \prod_{j=2}^{n} \beta_{j}-f^{\prime}(0) \prod_{j=2}^{n} \alpha_{j}>0$.

(iv)Quando $r=0, P(\lambda)+Q(\lambda)=0$ possue um número finito de raízes, já que é uma função polinomial.

(v)

$$
\begin{aligned}
F(y) & =|P(i y)|^{2}-|Q(i y)|^{2} \\
& =\left|(b+i y) \prod_{j=2}^{n}\left(\beta_{j}-i y\right)\right|^{2}-f^{\prime}(0)^{2}\left(\prod_{j=2}^{n} \alpha_{j}\right)^{2} \\
& =\left(b^{2}+y^{2}\right) \prod_{j=2}^{n}\left(\beta_{j}^{2}+y^{2}\right)-f^{\prime}(0)^{2} \prod_{j=2}^{n} \alpha_{j}^{2},
\end{aligned}
$$


a última passagem é obtida utilizando-se indução finita. Assim, $F(y)=0$ é um polinômio e, portanto, possue um número finito de raízes.

Podemos considerar dois casos:

Primeiro caso) $\quad b \prod_{j=2}^{n} \beta_{j}>-f^{\prime}(0)\left(\prod_{j=2}^{n} \alpha_{j}\right)$.

Como $\alpha_{j}, \beta_{j}, b,-f^{\prime}(0), y$ são todos não negativos, segue que

$$
\left(b^{2}+y^{2}\right) \prod_{j=2}^{n}\left(\beta_{j}^{2}+y^{2}\right)>b^{2} \prod_{j=2}^{n} \beta_{j}^{2}>f^{\prime}(0)^{2} \prod_{j=2}^{n} \alpha_{j}^{2},
$$

$\log$,

$$
F(y)=\left(b^{2}+y^{2}\right) \prod_{j=2}^{n}\left(\beta_{j}^{2}+y^{2}\right)-f^{\prime}(0)^{2} \prod_{j=2}^{n} \alpha_{j}^{2}>0,
$$

para todo $y>0$ e então $F(y)$ não possue solução. Pela conclusão a) do Teorema (2.2) segue que se a equação for estável em $r=0$ então a equação é estável para todo $r \geq 0$.

Segundo caso) $\quad b \prod_{j=2}^{n}\left(\beta_{j}\right)<-f^{\prime}(0) \prod_{j=2}^{n} \alpha_{j}$.

Neste caso, $F(y)=0$ possue uma única raiz positiva.

Usando indução e a regra do produto para derivadas, é fácil provar que:

$$
F^{\prime}(y)=2 y\left[\prod_{j=2}^{n}\left(\beta_{j}+y^{2}\right)+\left(b+y^{2}\right) \sum_{k=2}^{n} \prod_{\substack{j=2 \\ j \neq k}}^{n}\left(\beta_{j}^{2}+y^{2}\right)\right] .
$$

logo, $F^{\prime}(y)>0$, já que $y>0$ e $\left(b^{2}+y^{2}\right) \prod_{\substack{j=2 \\ j \neq k}}^{n}\left(\beta_{j}^{2}+y^{2}\right)>0$, para todo $k$.

Utilizando a parte b) do Teorema (2.2), concluímos que, um cruzamento ocorre, implicando em instabilidade e a estabilidade não pode voltar a ocorrer, já que nenhum outro cruzamento ocorre.

No capítulo 3, vimos as condições para garantirmos a estabilidade absoluta, e agora através delas vamos tentar encontrar condições sobre o modelo deste exemplo no caso $n=2$.

A primeira con dição afirma que $P(z)$ não possue zeros em $\operatorname{Re}(z) \leq 0$. Para verificarmos tal condição, suponhamos que $P(z)$ tenha uma solução $z_{0}=u+i v$, portanto $P\left(z_{0}\right)=0$, o que implica em

$$
(b+u+i v)\left(\beta_{2}+u+i v\right)=0,
$$


igualando partes real e imaginária, temos

$$
\begin{cases}b \beta_{2}+b u+u \beta_{2}+u^{2}-v^{2} & =0 \\ b v+2 u v+\beta_{2} v & =0\end{cases}
$$

Da última expressão temos duas possibilidades: $v=0$, ou $b+2 u+\beta_{2}=0$. Se esta última ocorrer, como $b, \beta_{2}>0$, segue que $u<0$ e assim a primeira condição está satisfeita. Se $v=0$, então da primeira expressão, ficamos com $u^{2}+u\left(b+\beta_{2}\right)+b \beta_{2}=0$. Resolvendo em $u$, obtemos

$$
u=\frac{-\left(b+\beta_{2}\right) \pm\left|b-\beta_{2}\right|}{2} .
$$

Se $b>\beta_{2}$, segue que $u_{1}=-\beta_{2}<0$ e $u_{2}=-b<0$.

Se $b<\beta_{2}$, segue que $u_{1}^{\prime}=-b<0$ e $u_{2}^{\prime}=-\beta_{2}<0$.

Se $b=\beta_{2}$, segue que $z_{0}=0$, o que não nos interessa.

Assim, concluímos que independente dos coeficientes, a primeira condição para termos estabilidade absoluta está sempre satisfeita.

Vejamos a segunda condição, ou seja, $|P(i y)|>|Q(i y)|$, para todo $0 \leq y<\infty$. Devemos ter :

$$
b^{2} \beta_{2}^{2}+y^{4}+y^{2}\left(b^{2}+\beta_{2}^{2}\right)>\alpha_{2}^{2}\left(f^{\prime}(0)\right)^{2} .
$$

Basta exigirmos que $b \beta_{2}>\left|\alpha_{2} f^{\prime}(0)\right|$. Portanto, esta é a única condição para termos estabilidade absoluta no modelo acima no caso $n=2$.

Exemplo 5.2. Vejamos um exemplo, cuja equação característica contém dois retardos e é definida da seguinte maneira:

$$
z+a e^{-T_{1} z}+b e^{-T_{2} z}=0
$$

onde $T_{1} \geq 0, T_{2} \geq 0$ e $a, b$ são constantes positivas. Note que esta equação tem a forma (2.2), logo podemos aplicar o Teorema (2.4), onde $\tau_{1}=T_{1}, T=T_{2}, P_{0}(z)=z$, $P_{1}(z)=a$ e $Q(z)=b$, portanto $P(z)=z+a e^{-T_{1} z}$ e $Q(z)=b$. É fácil ver que as hipóteses do Teorema (2.4) estão satisfeitas, pois $Q$ é constante. Assim, as conclusões deste teorema são válidas. Calculando $F(y)$, temos:

$$
F(y)=\left|i y+a\left(\cos T_{1} y-i \operatorname{sen} T_{1} y\right)\right|^{2}-b^{2}=a^{2}-b^{2}-2 a y \operatorname{sen} T_{1} y+y^{2}
$$

cuja derivada em relação a $y$ é dada por:

$$
\frac{1}{2} F^{\prime}(y)=y-a \operatorname{sen} T_{1} y-a T_{1} y \cos T_{1} y
$$


Analisemos a equação $F(y)=0$. Note que podemos escrevê-la da seguinte forma:

$$
2 a \operatorname{sen} T_{1} y=\frac{a^{2}-b^{2}}{y}+y .
$$

Denotaremos $g(y)=\frac{a^{2}-b^{2}}{y}+y$, para facilitar a notação.

Caso 1) $\quad 0<a=b$.

Neste caso $F(y)=0$ se resume em

$$
2 a \operatorname{sen} T_{1} y=y .
$$

Segue que $y=0$ é uma solução de $F(y)=0$, mas se substituirmos na equação característica, teremos $2 a=0$, contrário à hipótese. Portanto basta considerarmos os outros zeros da equação acima.

Derivando $2 a \operatorname{sen}\left(T_{1} y\right)=y$ em relação a $y$, temos

$$
2 a T_{1} \cos \left(T_{1} y\right)=1 \Rightarrow \cos \left(T_{1} y\right)=\frac{1}{2 a T_{1}} .
$$

Assim,

- No caso de $2 a T_{1} \leq 1$, temos $\cos \left(T_{1} y\right)=\frac{1}{2 a T_{1}} \geq 1$. Se $\cos \left(T_{1} y\right)=1$, obtemos $y=0$, o que não nos interessa, já que buscamos solução positiva. Segue que, nestas condições não existe raiz positiva de $F(y)=0$ e portanto nenhuma troca de estabilidade pode ocorrer conforme $T_{2}$ cresce.

- Se $2 a T_{1}>1$, concluímos que existe um número ímpar de raízes positivas de $F(y)=0$, que possibilitam trocas de estabilidade conforme $T_{2}$ cresce.

Para facilitar a discussão deste fato, façamos $T_{1}=1$. Daí, $F(y)=0$, se resume em

$$
\operatorname{sen}(y)=\frac{y}{2 a} .
$$

Como estamos no caso $2 a T_{1}>1$, então $a>\frac{1}{2}$. É necessário calcularmos os possiveis valores de $y$ que satisfaçam a igualdade acima. Para isto, vamos traçar os 
gráficos das funções sen $(y)$ e $\frac{y}{2 a}$.

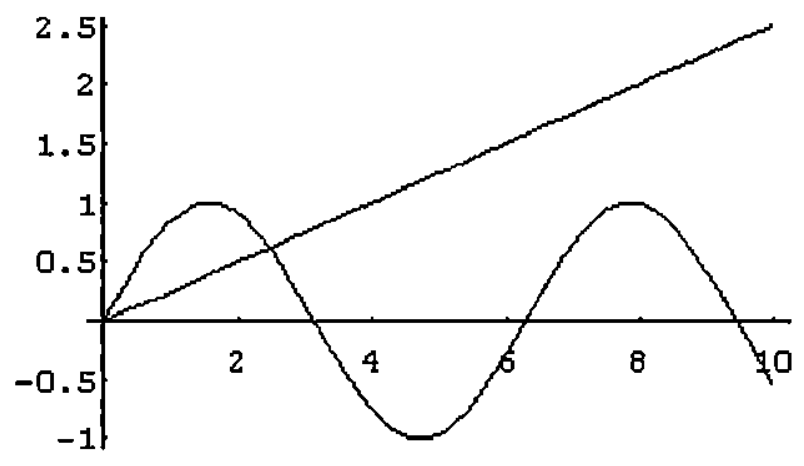

Figura 5.1

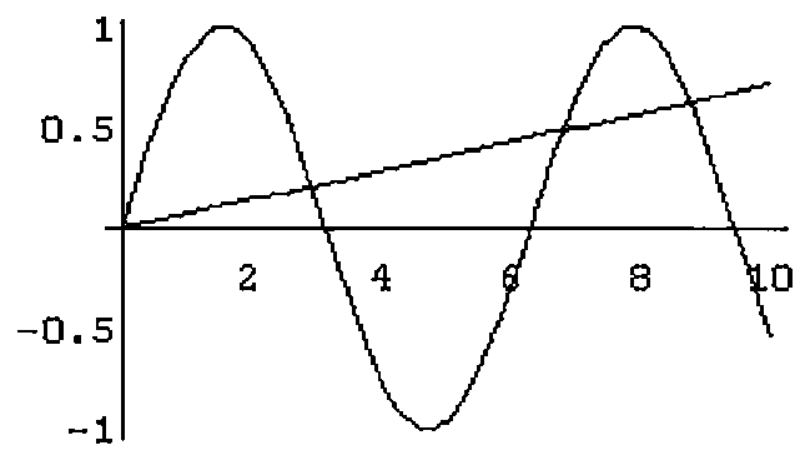

Figura 5.2

É fácil ver que, para valores pequenos de $a$, Figura 5.1, existe exatamente uma raiz positiva de $F(y)=0$ e de acordo com a teoria do Capítulo 2, caso particular para funções $P$ e $Q$ polinomiais, raízes somente podem cruzar o eixo imaginário do lado esquerdo para o direito quando $T_{2}$ cresce. Portanto a desestabilização ocorre e a estabilidade nunca volta a ocorrer.

Por outro lado, quando a cresce, Figura 5.2, existe um número ímpar de raízes imaginárias de $F(y)=0$, devido a periodicidade da função seno, e, portanto, apli- 
cando o Teorema (2.4) trocas de estabilidade podem ocorrer e existe um $T_{2}^{*}$ tal que para $T_{2}>T_{2}^{*}$ temos instabilidade.

Por exemplo, fixando $a=4$, temos que $F(y)=0$ implica sen $y=\frac{y}{8}$ e utilizando o software "Mathematica"obtemos três soluções positivas

$$
y_{1}=7.95732, \quad y_{2}=7.49775, \quad \text { e } \quad y_{3}=2.7859 .
$$

Então, $y_{1}>y_{2}>y_{3}>0$, e (2.6) implica que

$$
\cos \left(T_{2} y\right)=\frac{-\left(a^{2} \cos \left(y_{1}\right)+0\right)}{a^{2}}=-\cos y_{1} .
$$

Logo,

$$
T_{2} y_{1}=1.467458 \Rightarrow T_{2}=0.184512
$$

assim, para $y_{1}$ temos a seguinte sequência:

$$
T_{m, 1}=0.184512+\frac{2 \pi m}{y_{1}}=0.184512, \quad 0.974123, \quad 1.763733, \quad 2.55334,
$$
$3.34295, \cdots$.

Repetindo as contas para $y_{2}$ e para $y_{3}$, obtemos

$$
3.609052, \cdots \text {, }
$$

$$
T_{m, 2}=0.2570142+\frac{2 \pi m}{y_{2}}=0.2570142, \quad 1.095024, \quad 1.93303, \quad 2.771043,
$$

$$
T_{m, 3}=0.127677+\frac{2 \pi m}{y_{3}}=0.127677, \quad 2.38303, \quad 4.6384, \quad 6.89373,
$$

Portanto, o primeiro cruzamento se dá em $y_{3}$, o segundo em $y_{1}$ e o terceiro em $y_{2}$. Para $T_{2}=0$, a equação (5.5) se reduz a

$$
z+a+a^{-T_{1} z}=0
$$

e assim $F(y)=y^{2}$, a qual não possue soluções positivas, e portanto nenhuma troca de estabilidade é possível ocorrer. Quando $T_{1}=0$ temos $z+2 a=0$. Para $z=x+i y$ solução vale $x+i y+2 a=0$ e portanto $x=-2 a<0$, o que implica em estabilidade. Em particular, para $T_{1}=1$ e $T_{2}=0$ temos estabilidade. E de acordo com a teoria estudada no Capítulo 2, segue que a equação é estável para $0 \leq T<T_{2}^{*}=$ 
$0.127677=T_{2}^{*}$ e para $T \geq T_{2}^{*}$ a equação é instável.

Caso 2) $\quad 0<a<b$.

Consideremos a função $g(y)=\frac{a^{2}-b^{2}}{y}+y$.

Para $y>0$, segue que

$$
g^{\prime}(y)=\frac{b^{2}-a^{2}}{y^{2}}+1>0, \quad g^{\prime \prime}(y)=\frac{2 y\left(a^{2}-b^{2}\right)}{y^{4}}<0,
$$

logo, $g$ é uma função monótona crescente cujo gráfico é côncavo para baixo em todo ponto $(y, g(y))$. E ainda, $g(y)=0$ se, e só se, $y=\left(b^{2}-a^{2}\right)^{\frac{1}{2}} \mathrm{e}$

$$
\lim _{y \rightarrow \infty} g(y)=\infty, \quad \lim _{y \rightarrow 0} g(y)=-\infty .
$$

Através desta análise, podemos traçar um esboço do gráfico da função $g$. Como $F(y)=0$ implica $2 a \operatorname{sen}\left(T_{1} y\right)=g(y)$, se considerarmos $a=1, b=2$ e $T_{1}=1$, temos geometricamente:

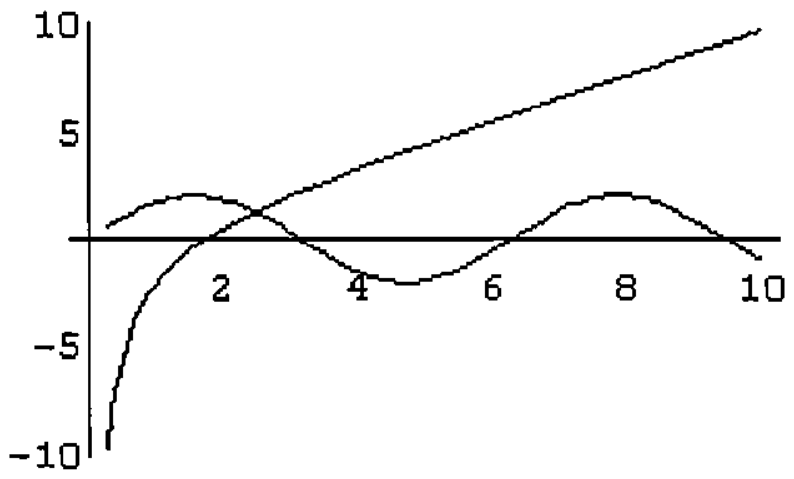

Figura 5.3 
Busquemos os valores de $y$ tais que $g(y) \leq 2 a$,

$$
\frac{a^{2}-b^{2}}{y}+y \leq 2 a \Rightarrow a^{2}-b^{2}+y^{2}-2 a y \leq 0
$$

e resolvendo a equação do segundo grau envolvida temos as soluções $y_{1}=a+b \mathrm{e}$ $y_{2}=a-b<0$ a qual não nos interessa. Portanto $g(y) \leq 2 a$ implica $0 \leq y \leq a+b$.

Provemos que os gráficos de $g(y)$ e $2 a \operatorname{sen}\left(T_{1} y\right)$ se interceptam em um número ímpar de vezes. Vejamos quais os pontos onde

$$
2 a \operatorname{sen}\left(T_{1} y\right)=0 \text {. }
$$

Isto implica que sen $\left(T_{1} y\right)=0$, e assim $y=n \frac{\pi}{T_{1}}$. É claro que o gráfico de $g(y)$ corta o eixo $y$ antes de $y_{0}=\pi / T_{1}$, já que $g\left(y_{0}\right)<0$.

Sabemos que $-2 a \leq 2 a \operatorname{sen}\left(T_{1} y\right) \leq 2 a$, então $2 a \operatorname{sen}\left(T_{1} y\right)=2 a$ se, e só se, $\operatorname{sen}\left(T_{1} y\right)=1$, o que implica $T_{1} y=\frac{\pi}{2}+2 n \pi$ então $y=\frac{\pi(1+4 n)}{2 T_{1}}$.

Se $g$ encontrar o gráfico de $2 a \operatorname{sen}\left(T_{1} y\right)$ em um único ponto, como na Figura (5.3), então a conclusão está satisfeita. Agora, se o gráfico da função $g$ interceptar mais de uma vez o gráfico da função $2 a \operatorname{sen}\left(T_{1} y\right)$, devido a periodicidade da função seno o número deve ser ímpar, como na figura abaixo.

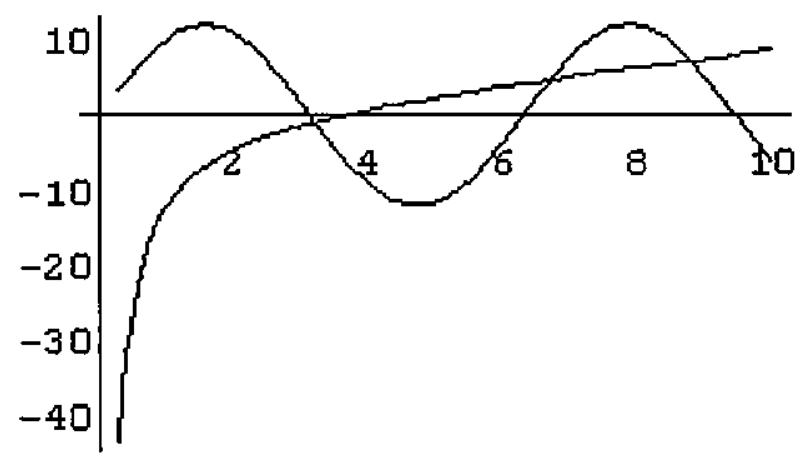

Figura 5.4

Precisamos somente mostrar que o gráfico da função $g(y)$ não pode tangenciar o gráfico da função seno. Assim, perguntamos:

Pode ocorrer $g\left(\frac{5 \pi}{2 T_{1}}\right)=2 a ?(\mathrm{n}=1)$ 
Se essa igualdade ocorrer, então, consequentemente teremos

$$
\begin{gathered}
4 T_{1}^{2}\left(a^{2}-b^{2}\right)+(5 \pi)^{2}=2 a 10 \pi T_{1} \Rightarrow b^{2}=a^{2}+\frac{(5 \pi)^{2}}{4 T_{1}^{2}}-\frac{5 a \pi}{T_{1}} \\
b^{2}=\left(a-\frac{5 \pi}{2 T_{1}}\right)^{2} \Rightarrow b=a-\frac{5 \pi}{2 T_{1}} \Rightarrow b<a
\end{gathered}
$$

o que é contraditório à hipótese assumida neste caso. Se tomarmos qualquer outro ponto do tipo $\frac{\pi(1+4 n)}{2 T_{1}}$ teremos a mesma conclusão, assim estes casos (de tangência) não podem ocorrer e de fato, temos um número ímpar de soluções de $F(y)=0$.

Quando $T_{2}=0$ a equação (5.5) se reduz a

$$
z+b+a e^{-T_{1} z}=0
$$

e nesta situação temos que $F(y)=0$ implica $y^{2}=a^{2}-b^{2}<0$, e portanto $F(y)=0$ não tem solução. Quando $T_{1}=0$, ficamos com $z+a+b=0$. Se $z=u+i v$ for uma solução, então

$$
u+i v+a+b=0 \Rightarrow v=0, \quad u=-a-b<0,
$$

e assim temos estabilidade, sempre que $0<a<b$. Conclui-se que a equação é estável para $T_{2}=0$ e para qualquer $T_{1}$.

Portanto, se existir uma única raiz de $F(y)=0$ então todas as raízes que cruzam o eixo imaginário são desestabilizadoras, logo existe um $T_{2}^{*}$, dependendo de $T_{1}$, tal que a equação é estável para $0 \leq T_{2}<T_{2}^{*}$ e instável para $T_{2}>T_{2}^{*}$, onde $T_{2}^{*}$ é o instante em que o primeiro cruzamento ocorre.

Se existir mais de uma solução, por exemplo, se fixarmos $T_{1}=1, a=3$ e $b=6$, obtemos três soluções positivas de $F(y)=0$,

$$
y_{1}=8.28698, \quad y_{2}=6.76327, \quad \text { e } \quad y_{3}=3.75263 \text {. }
$$

Com $y_{1}>y_{2}>y_{3}>0$, e usando (2.6), obtemos:

$$
\cos \left(T_{2} y\right)=\frac{-a \cos y}{b}=-\frac{\cos y}{2} .
$$

Logo, para $y_{1}$ temos

$$
T_{2} y_{1}=1.313922 \Rightarrow T_{2}=0.1566621
$$


e consequentemente obtemos a seguinte seqüência:

$$
T_{m, 1}=0.1566621+\frac{2 \pi m}{y_{1}}=0.1566621, \quad 0.905822, \quad 1.654981, \quad 2.404140,
$$

$3.1532999, \cdots$.

Repetindo as contas para $y_{2}$ e para $y_{3}$, obtemos

$$
T_{m, 2}=0.3002+\frac{2 \pi m}{y_{2}}=0.3002, \quad 1.22922, \quad 2.158232, \quad 3.087248,
$$

$4.0163, \cdots$,

$$
T_{m, 3}=0.30615+\frac{2 \pi m}{y_{3}}=\quad 0.30615, \quad 1.980492, \quad 3.654834, \quad 5.329176,
$$

Segundo a teoria do Capítulo 2, para $T_{1}=1, a=3$ e $b=6$, concluímos que:

1) para $0 \leq T_{2}<0.1566621$, teremos estabilidade;

2) para $0.1566621 \leq T_{2}<0.3002$ teremos instabilidade;

3) para $0.3002 \leq T_{2}<0.30615$ teremos estabilidade,

4) para $T_{2} \geq 0.30615=T_{2}^{*}$ teremos instabilidade.

Vale observar que, a análise da estabilidade da equação (5.5) se torna mais complexa conforme o número de raízes de $F(y)=0$ aumenta. Mas, pelo Teorema (2.4), podemos garantir que pode ocorrer um número finito de trocas de estabilidade e existe um $T_{2}^{*}$, dependendo de $T_{1}$, tal que a equação é instável para todo $T_{2}>T_{2}^{*}$.

Exemplo 5.3. Consideremos o seguinte sistema de equações diferenciais com retardamento:

$$
\left\{\begin{array}{l}
x^{\prime}(t)=f(x(t), x(t-\tau), y(t), y(t-\xi)), \\
y^{\prime}(t)=g(x(t), x(t-\tau), y(t), y(t-\xi)),
\end{array}\right.
$$

onde $f, g: \mathbb{R}^{4} \rightarrow \mathbb{R}$.

Tais sistemas surgem em vários problemas, como por exemplo modelos de interações de populações onde o retardo aparece no processo de nascimento. Também nos modelos de presa e predador que veremos ainda neste capítulo. Por exemplo, veja [7].

Suponhamos que as funções $f$ e $g$ tenham derivadas parciais de primeira ordem contínuas com respeito aos argumentos e que existam únicos $x^{*}>0, y^{*}>0$ tal que $f\left(x^{*}, x^{*}, y^{*}, y^{*}\right)=g\left(x^{*}, x^{*}, y^{*}, y^{*}\right)=0$. Seja $f_{i}, i=1,2,3,4$, a derivada parcial de $f$ com respeito ao seu i-ésimo argumento calculada em $P^{*}\left(x^{*}, x^{*}, y^{*}, y^{*} z\right.$ ) (idem para $g_{i}$. 
Procurando soluções da forma $u(t)=c_{1} e^{\lambda t}, v(t)=c_{2} e^{\lambda t}$, com $c_{1}$ e $c_{2}$ constantes, temos a seguinte equação característica:

$$
\lambda^{2}+a \lambda+b+(c \lambda+p) e^{-\lambda \tau}+(d \lambda+q) e^{-\lambda \xi}+r e^{-(\tau+\xi) \lambda}=0,
$$

onde

$$
\begin{gathered}
a=-\left(f_{1}+g_{3}\right), \quad b=f_{1} g_{3}-f_{3} g_{1}, \quad c=-f_{2}, \quad d=-g_{4}, \\
p=f_{2} g_{3}-f_{3} g_{2}, \quad q=f_{1} g_{4}-f_{4} g_{1}, \quad r=f_{2} g_{4}-f_{4} g_{2} .
\end{gathered}
$$

Fazendo $z=\lambda, T_{1}=\tau, T_{2}=\xi$ e $a=b=c=d=r=0$, temos a seguinte equação característica:

$$
z^{2}+p e^{-T_{1} z}+q e^{-T_{2} z}=0
$$

e suponhamos que $p$ e $q$ sejam constantes positivas. É fácil ver que as condições do Teorema (2.4) estão todas satisfeitas. Assim, podemos escrever:

$$
\begin{gathered}
F(y)=\mid-y^{2}+p\left(\cos \left(T_{1} y\right)-\left.i \operatorname{sen}\left(T_{1} y\right)\right|^{2}-q^{2}=y^{4}+p^{2}-q^{2}-2 p y^{2} \cos \left(T_{1} y\right)=0,\right. \\
2 p \cos \left(T_{1} y\right)=\frac{p^{2}-q^{2}}{y^{2}}+y^{2} .
\end{gathered}
$$

Vamos discutir alguns casos:

Caso 1) $0<p=q$.

A equação $F(y)=0$ se resume em :

$$
2 p \cos \left(T_{1} y\right)=y^{2} .
$$

Derivando, temos $-p T_{1} \operatorname{sen}\left(T_{1} y\right)=y$, e, derivando novamente $\cos T_{1} y=\frac{-1}{p T_{1}^{2}}$.

-Se $p T_{1}^{2}<1$, então $F(y)=0$ não possue solução e nenhuma troca de estabilidade é possível ocorrer conforme $T_{2}$ cresce.

-Se $p T_{1}^{2} \geq 1$, então existe um número ímpar de soluções positivas de $F(y)=0$. Novamente, para facilitar a discussão, façamos $T_{1}=1$ e assim temos $\cos (y)=\frac{y^{2}}{2 p} \mathrm{e}$ 
portanto $p \geq 1$ e $y^{2} \leq 2 p$. Note que, se $y=\sqrt{2 p}$ temos que $\cos y=1$, o que implica $y=0$ e $p=0$ o que não nos interessa.

Fixando $T_{1}=1$, para $1 \leq p<2 \pi^{2}$, temos uma única solução positiva, como mostra a figura abaixo:

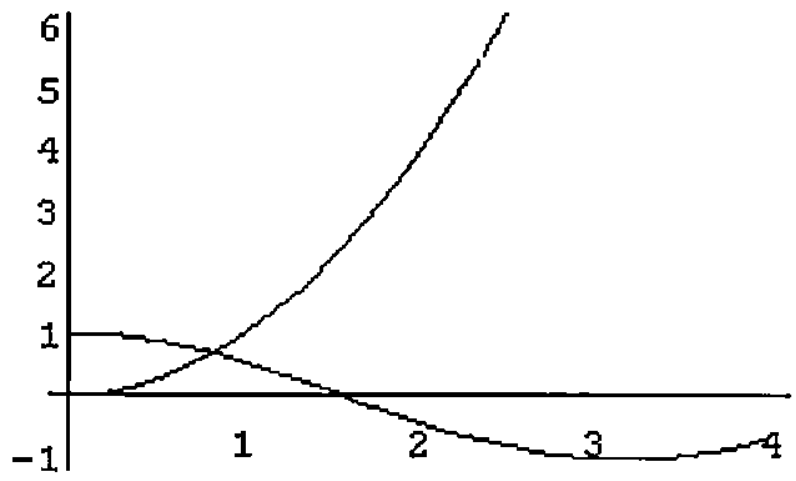

Figura 5.5

Já, para $p=2 \pi^{2}$, temos que $y=\sqrt{2 p}$ é solução e portanto recaímos na discussão anterior.

Para $2 \pi^{2}<p<8 \pi^{2}$ temos três interseções. Devido a periodicidade da função cosseno, teremos sempre número ímpar de soluções positivas conforme $p$ varia. Logo, para $a$ pequeno, temos uma única raiz positiva de $F(y)=0$ e de acordo com a teoria, raízes somente cruzam do lado esquerdo para o direito.

Quando $T_{2}=0$ a equação (5.7) se reduz a $z^{2}+p+p^{-T_{1} z}=0$. Pela análise realizada no Capítulo 2 temos instabilidade, para qualquer $T_{1} \geq 0$. Em particular, para $T_{2}=0$ e $T_{1}=1$ temos instabilidade.

Para $p<18,5$, a equação é instável para todo $T_{2} \geq 0$ e $T_{1}=1$. Se $p>18.5$ existe um número ímpar de soluções positivas de $F(y)=0$. Para exemplificar, façamos $p=20$. Daí, $F(y)=0$ implica em $\cos (y)=\frac{y^{2}}{40}$. Obtemos três soluções positivas

$$
y_{1}=6.32215, \quad y_{2}=5.62473, \quad \text { e } y_{3}=1.5135 \text {. }
$$

Então, $y_{1}>y_{2}>y_{3}>0$, usando (2.6), obtemos:

$$
\cos \left(T_{2} y\right)=\frac{-p\left(p \cos y-y^{2}\right)}{p^{2}}=-\cos y+\frac{y^{2}}{20} \text {. }
$$


Geometricamente, temos

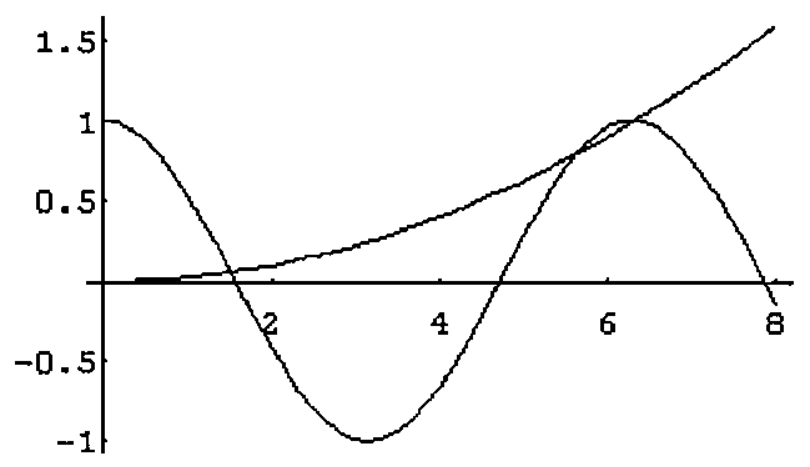

Figura 5.6

Logo, para $y_{1}$ temos

$$
T_{2} y_{1}=0.039040 \Rightarrow T_{2}=0.0061751
$$

e consequentemente temos a seguinte seqüência:

$$
T_{m, 1}=0.0061751+\frac{2 \pi m}{y_{1}}=0.0061751, \quad 1.000012, \quad 1.9938487, \quad 2.98769,
$$
$3.981522, \cdots$.

Repetindo as contas para $y_{2}$ e para $y_{3}$, obtemos

$$
T_{m, 2}=0.1170636+\frac{2 \pi m}{y_{2}}=0.1170636, \quad 1.23413, \quad 2.3511923, \quad 3.468257,
$$
$4.585321, \cdots$,

$$
T_{m, 3}=0.9999973+\frac{2 \pi m}{y_{3}}=0.9999973, \quad 5.151425, \quad \cdots .
$$

Segundo a nossa teoria, segue que $T_{2}^{*}=0$, ou seja a equação é instável para todo $T_{2} \geq 0$ e $T_{1}=1$.

Caso 2) $\quad 0<p<q$

Para facilitar nossa análise, consideremos a função $g(y)=\frac{p^{2}-q^{2}}{y^{2}}+y^{2}$. 
Para $y>0$, temos que

$$
g^{\prime}(y)=\frac{2\left(q^{2}-p^{2}\right)}{y^{3}}+2 y>0, \quad g^{\prime \prime}(y)=\frac{6\left(p^{2}-q^{2}\right)}{y^{4}}+2
$$

logo, $g$ é uma função monótona crescente e se $0<y^{4}<3\left(q^{2}-p^{2}\right)$ o gráfico é côncavo para baixo. E ainda, $g(y)=0$ se, e só se, $y=\left(q^{2}-p^{2}\right)^{\frac{1}{4}} \mathrm{e}$

$$
\lim _{y \rightarrow \infty} g(y)=\infty, \quad \lim _{y \rightarrow 0} g(y)=-\infty
$$

Agora, podemos traçar um esboço do gráfico da função $g . F(y)=0$ implica que

$$
2 p \cos \left(T_{1} y\right)=g(y)
$$

Se considerarmos $p=5, q=6$ e $T_{1}=1$, temos geometricamente:

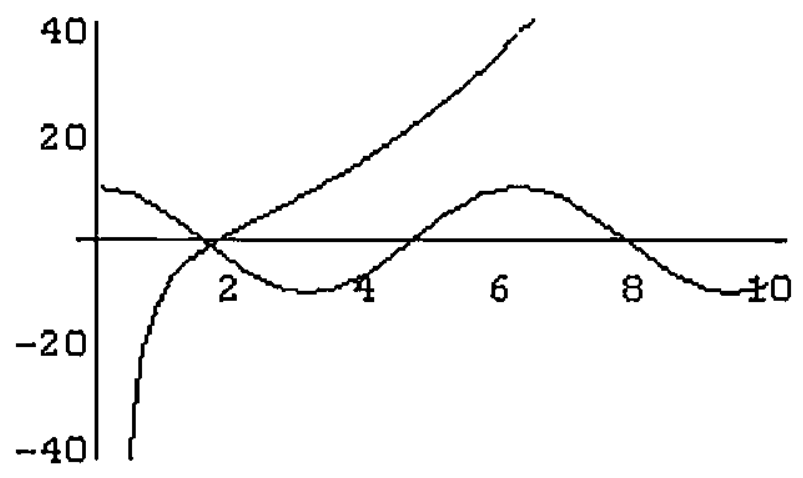

Figura 5.7

Neste caso, a equação $F(y)=0$ possue apenas uma raiz positiva e como a equação é instável para $T_{2}=0$, segue que a equação é instável para todo $T_{2} \geq 0$.

Se $F(y)=0$ possuir mais que uma raiz a análise se torma mais complexa. Para exemplificar, façamos $T_{1}=1, p=50$ e $q=120$, e segue que existe três soluções positivas de $F(y)=0$, dadas por:

$$
y_{1}=12.894, \quad y_{2}=11.3821, \quad e \quad y_{3}=8.75678
$$

Então, $y_{1}>y_{2}>y_{3}>0$, usando (2.6), obtemos: 


$$
\cos \left(T_{2} y\right)=\frac{-\left(p \cos y-y^{2}\right) q}{q^{2}}=-\frac{50 \cos y}{120}+\frac{y^{2}}{120}
$$

Geometricamente, temos

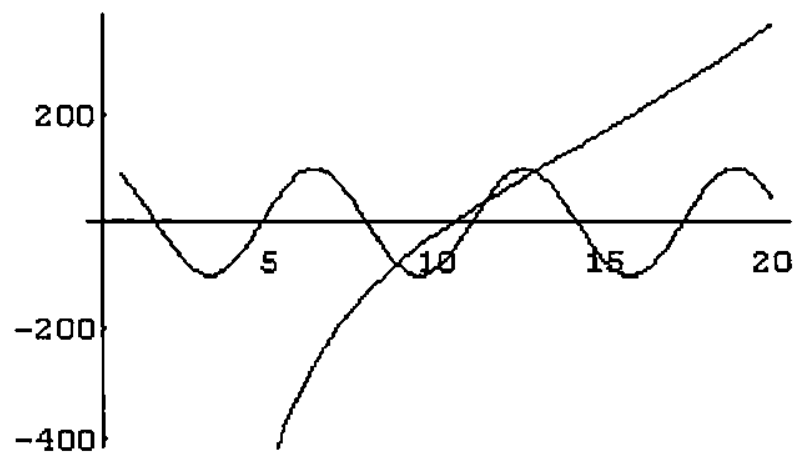

Figura 5.8

Logo, para $y_{1}$ temos

$$
T_{2} y_{1}=0.1345858 \Rightarrow T_{2}=0.010438
$$

e obtemos a seguinte seqüência:

$$
T_{m, 1}=0.010438+\frac{2 \pi m}{y_{1}}=\quad 0.010438, \quad 0.9637966, \quad 1.4456949, \quad \cdots \text {. }
$$

Repetindo as contas para $y_{2}$ e para $y_{3}$, obtemos

$$
\begin{aligned}
& T_{m, 2}=0.03481+\frac{2 \pi m}{y_{2}}=0.03481,1.126476, \ldots, \\
& T_{m, 3}=0.02981111+\frac{2 \pi m}{y_{3}}=0.02981111, \ldots .
\end{aligned}
$$

Segundo a teoria do Capítulo 2, neste caso $T_{1}=1, p=50$ e $q=120$ teremos, para qualquer $T_{2} \geq 0$, instabilidade. 
Exemplo 5.4. Consideremos um modelo do tipo presa-predador, veja [7], com um retardo (este retardo se deve ao fato da caça por predadores só ocorrer quando este estiver preparado para caçar). O modelo apresenta a seguinte forma:

$$
\left\{\begin{array}{l}
x^{\prime}(t)=x(t) g(x(t))-y^{m}(t-\xi) p(x(t)) \\
y^{\prime}(t)=-s y(t)+c y^{m}(t) p(x(t-\tau))
\end{array}\right.
$$

onde $g(x)$ é a taxa de crescimento da presa $x, p(x)$ é a função reação do predador e $0 \leq m \leq 1$ é o parâmetro de interferência mútua.

Inicialmente vamos linearizar o sistema acima, já que este é um sistema não linear. Se denotarmos $\left(x^{*}, y^{*}\right)$ o ponto equilíbrio teremos, pela mudança,

$$
u(t)=x(t)-x^{*}, \quad v(t)=y(t)-y^{*}
$$

o seguinte sistema:

$$
\frac{d}{d t}\left(\begin{array}{l}
u \\
v
\end{array}\right)=\left(\begin{array}{cc}
H & 0 \\
0 & R
\end{array}\right)\left(\begin{array}{l}
u(t) \\
v(t)
\end{array}\right)+\left(\begin{array}{cc}
0 & N \\
Q & 0
\end{array}\right)\left(\begin{array}{l}
u(t-\tau) \\
v(t-\xi)
\end{array}\right)
$$

onde

$$
\begin{aligned}
H & =x^{*} g_{x}\left(x^{*}\right)+g\left(x^{*}\right)-y^{* m} p_{x}\left(x^{*}\right)<0 \\
R & =c(m-1) y^{* m-1} p\left(x^{*}\right)<0 \\
N & =-m y^{* m-1} p\left(x^{*}\right)<0 \\
Q & =c y^{* m} p_{x}\left(x^{*}\right)>0
\end{aligned}
$$

Assim, se chamarmos $a=-(H+R)>0, b=H R>0$ e $r=-N Q>0$, temos a seguinte equação característica:

$$
\lambda^{2}+a \lambda+b+r e^{-(\tau+\xi) \lambda}=0
$$

e portanto, $P(\lambda)=\lambda^{2}+a \lambda+b$ e $Q(\lambda)=r$, ou seja, $Q$ é uma função constante.

Fazendo $\tau+\lambda=T$, podemos utilizar a análise já realizada na Segunda Equação do Capítulo 2 e assim, obtermos as seguintes conclusões:

(1) Se $b^{2}<r^{2}$, a estabilidade da solução nula é perdida em um certo $T_{0}$ e 
a instabilidade prevalece para todo $T>T_{0}$;

(2) Se $b^{2}>r^{2}$, um número finito de trocas de estabilidade ocorre e para $T$ suficientemente grande a instabilidade prevalece;

(3) Se $b^{2}=r^{2}$, a estabilidade da solução nula é perdida em um certo $T_{0} \mathrm{e}$ a instabilidade prevalece para todo $T>T_{0}$.

Olhando o retardo $\tau+\xi=T$ como um único retardo, passemos a analisar o modelo quanto à estabilidade absoluta, estudada no Capítulo 3. Como $P(\lambda)=$ $\lambda^{2}+a \lambda+b$ e $Q(\lambda)=r$, é fácil ver que as condições do Teorema (3.1) estão satisfeitas. A condição $P(\lambda) \neq 0$ para $\lambda=u+i v$ em $\operatorname{Re}(\lambda) \geq 0$, é calculada da seguinte maneira:

$$
(u+i v)^{2}+a(u+i v)+b=0,
$$

igualando partes real e imaginária

$$
\begin{cases}u^{2}-v^{2}+a u+b & =0, \\ 2 u v+a v & =0 .\end{cases}
$$

Da última equação segue que, $v=0$ ou $u=-a / 2<0$. Se $v=0$, então, de (5.11), temos que $u^{2}+a u+b=0$, o que implica $u=\frac{-a \pm \sqrt{a^{2}-4 b}}{2}$.

Assim, para que $u<0$, devemos impor que $a<0$ e também $b=v^{2}+a^{2} / 4>0$ independente de $a$. Concluímos que, para $P(z) \neq 0$, devemos ter $a, b>0$.

Agora vejamos a condição $|Q(i y)|<|P(i y)|$. Como $P(i y)=-y^{2}+b+a y i$ e $Q(i y)=r$, devemos ter

$$
|Q(i y)|^{2}=r^{2}<\left(b-y^{2}\right)^{2}+a^{2} y^{2}=|P(i y)|^{2}
$$

o que equivale a impor que $y^{4}+\left(a^{2}-2 b\right) y^{2}+\left(b^{2}-r^{2}\right)>0$.

Pela Observação (3.1), devemos ter $b^{2}>r^{2}, a^{2}>2 b$ e $\Delta \geq 0$, o que implica $a^{4}+4 r^{2} \geq 4 a^{2} b$, ou $b^{2}>r^{2}$ e $\Delta<0$, o que implica $a^{4}+4 r^{2}<4 a^{2} b$.

Conclui-se que a solução da equação associada a (5.10) possue solução absolutamente estável se, e só se, $a>0, b>0, b^{2}>r^{2}$ e $a^{4}+4 r^{2}<4 a^{2} b$, ou $a>0, b>0$, $b^{2}>r^{2}, a^{2}>2 b$ e $a^{4}+4 r^{2} \geq 4 a^{2} b$. 
Exemplo 5.5. No estudo do reflexo da luz na pupila humana, realizado em [14] e em [19] surge a seguinte equação diferencial linear com retardamento:

$$
\frac{d a}{d t}+\alpha a(t)=\frac{\gamma}{\beta A^{*}} a(t-\tau),
$$

onde $\gamma, \alpha, A^{*}$ e o retardo $\tau$ são constantes positivas e $\beta$ é uma constante não negativa.

Buscando solução de (5.12) da forma $a(t)=c e^{t \lambda}$, obtemos a seguinte equação característica:

$$
\lambda+\alpha-\frac{\gamma}{\beta A^{*}} e^{-\lambda \tau}=0 .
$$

Neste caso $Q(\lambda)=C=-\frac{\gamma}{\beta A^{*}}>0$. Assim, é fácil verificar que as condições do Teorema (2.2) estão satisfeitas.

E como $F(y)=0$ implica em $y^{2}=C^{2}-\alpha^{2}$, temos duas possibilidades a serem consideradas:

(i) $C<\alpha$. Então, $F(y)=0$ não possue solução positiva. Segue, do Teorema (2.2) parte a) que a equação será estável para todo retardo $\tau \geq 0$, já que, neste caso, a equação é estável em $\tau=0$. Portanto a equação (5.12) será absolutamente estável se $-\frac{\gamma}{\beta A^{*}}<\alpha$.

(ii) $C>\alpha$. Neste caso, $F(y)=0$ possue uma única solução positiva, dada por $y_{0}=\sqrt{C^{2}-\alpha^{2}}$. Segundo a teoria estudada, os cruzamentos ocorrem da esquerda para a direita, implicando em instabilidade. Como a equação é instável para $\tau=0$, segue que a equação será instável para todo retardo $\tau \geq 0$.

Exemplo 5.6. O modelo abaixo tem aplicação em Hematologia. A presença de um retardo no controle da produção de células do sangue pela concentração de células do sangue periférico varia de pessoa para pessoa num período que pode ser de 17 a 28 dias.

Seja $y(t)$ a concentração de células circulantes (célula/kilo) e suponhamos que as células são retiradas aleatoriamente da circulação a uma taxa $\gamma$ proporcional à sua concentração, e que o fluxo (células/kilo/dia) dependa de $y(t)$ no instante $t-\tau$. Assim, temos a seguinte equação não linear: 


$$
\dot{y}(t)=-\gamma y(t)+\delta(y(t-\tau)) .
$$

Um dos modelos mais aceitos para a função $\delta$ é o seguinte:

$$
\delta(y(t-\tau))=\frac{\beta_{0} \theta^{n} y(t-\tau)}{\theta^{n}+y^{n}(t-\tau)}
$$

onde $n>1, \theta>0$ e $\beta_{0} \in \mathbb{R}$ são parâmetros escolhidos para concordar com os dados experimentais. Logo, de (5.14) temos

$$
\dot{y}(t)=-\gamma y(t)+\beta_{0} \frac{\theta^{n} y(t-\tau)}{\theta^{n}+y^{n}(t-\tau)} .
$$

Como a equação (5.15) não é linear, primeiramente precisamos linearizá-la em torno do equilíbrio $y^{*}$. Para isto, façamos a substituição $x(t)=y(t)-y^{*}$. De (5.15) obtemos:

$$
\dot{x}(t)=-\gamma\left(x(t)+y^{*}\right)+\frac{\beta_{0} \theta^{n}\left[x(t-\tau)+y^{*}\right]}{\theta^{n}+\left[x(t-\tau)+y^{*}\right]^{n}}
$$

donde, segue que

$$
\dot{x}(t)=-\gamma x(t)+\frac{\beta_{0} \theta^{n} x(t-\tau)\left[\theta^{n}+(1-n) y^{* n}\right]}{\left[\theta^{n}+y^{* n}\right]^{2}} .
$$

Logo, a equação característica associada tem a forma:

$$
\lambda+\gamma-\frac{\beta_{0} \theta^{n}}{\left[\theta^{n}+y^{* n}\right]^{2}}\left[\theta^{n}+(1-n) y^{* n}\right] e^{-r \lambda}=0
$$

A equação (5.16) possue a mesma forma que (5.13), assim o procedimento para analisar as trocas de estabilidade é similar ao anterior. 


\section{Bibliografia}

[1] R. Bellman, K. L. Cooke, Differential-Difference Equations, Academic Press, New York, London, (1963).

[2] F.Brauer, Absolute Stability in Delay Equations, J. Diff. Eq., 69 (1987), 185191.

[3] K.L. Cooke, P. van den Driessche, On zeroes of some transcendental equations, Funkcialaj Ekvacioj, 29 (1986), 77-90.

[4] K. L. Cooke, Z. Grossman, Discrete delay, distributed delay and stability switches, J. Math. Anal. and Appl., 86 (1982), 592-627.

[5] K. L. Cooke, J. Turi, Stability, instability in delay equations modeling human respiration, J. Math Biol., 32 (1994), 535-543.

[6] J.B. Conway, Functions of One Complex Variable, Springer-Verlag, New York, Berlin, (1978).

[7] H. I. Freedman, V. Sree Hari Rao, Stability criteria for a system involving two time delays, J. Appl. Math., 46 (1986), 552-560.

[8] S.M.S. Godoy, Existência de soluções periódicas, estabilidade e aplicações de uma classe de equações diferenciais retardadas autônomas não lineares, Tese de Doutorado, ICMSC-USP, São Carlos (1989).

[9] B. C. Goodwin, Oscillatory behavior in enzymatic control processes, Adv. Enzyme Reg., 3 (1965), 425-439.

[10] B. C. Goodwin, Temporal Organization in Cells, Academic Press, New York, (1963).

[11] J. K. Hale, Functional Differential Equations, Springer-Verlag, New York, Berlin, (1977).

[12] H. M. Hethcote, M. A. Lewis and P. Driessche, An epidemiological model with a delay and a nonlinear incidence rate, J. Math. Biology, 27 (1989), 49-64. 
[13] M. S. Lee, C. S. Hsu, On the $\tau$-decomposition method os 'stability analysis for retarded dynamical systems, SIAM J. Control., 7 (1969), 249-259.

[14] A. Longtin, J. G. Milton, Modelling autonomous oscilations in the human pupil light reflex using nonlinear delay-differential equations, Bull. Math. Biology, 51 (1989), 605-624.

[15] J. M. Mahaffy, A test for stability of linear differential delay equations, Quarterly Appl. Math., 40 (1982), 193-202.

[16] J. M. Mahaffy, Periodic solutions for certain protein synthesis models, J. Math. Anal. and Appl., 74 (1980), 72-105.

[17] R. M. May, Nonlinear phenomena in ecology and epidemiology, Nonlinear Dinamics, Annals New York Academy of Sciences, 357 (1980), 267-281.

[18] R. D. Nussbaum, A. J. B. Potter, Cyclic Differential Equations and Period Three Solutions os Differential -Delay Equations, J. Diff. Eq., 46(1982), 379408.

[19] M. R. Weissmann, Existência de Soluções Periódicas para uma classe de Equações Diferenciais Funcionais Retardadas e Aplicaçōes, Dissertação de Mestrado, ICMSC-USP, São Carlos (1997). 